\title{
Electrochemical, Manganese-assisted Carbon-Carbon Bond Formation between $\beta$-Keto Esters and Silyl Enol Ethers
}

\author{
Julia Strehl and Gerhard Hilt*
}

Supporting Information

Table of contents

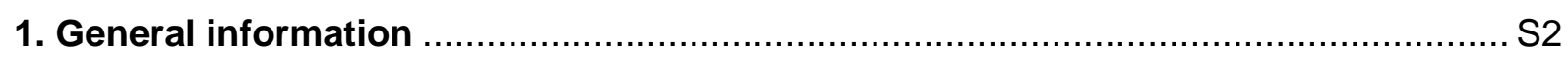

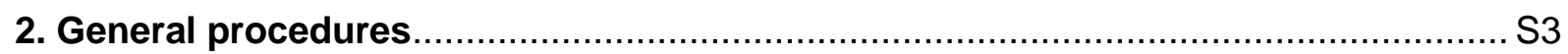

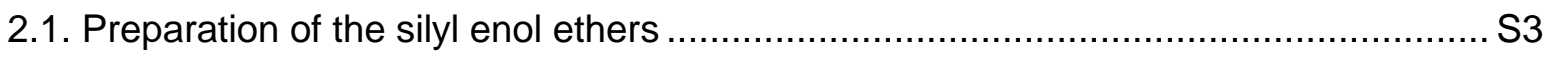

2.2. Electrochemical preparation of the diketoesters .................................................... 3

3. Optimization of the reaction conditions ………….............................................

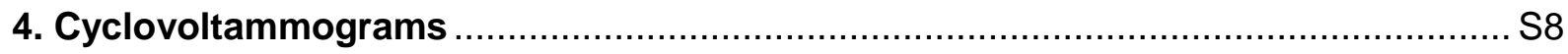

5. Synthesis of the silyl enol ethers ……...........................................................

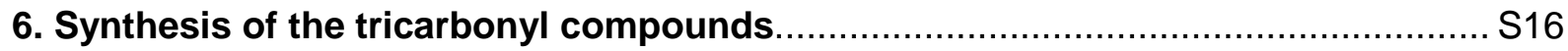

7. Synthesis of the Tris(pyridine-2-ylmethyl)amine (TPA) ligand: ............................. S26

8. Synthesis of the manganese-TPA-complex........................................................ 227

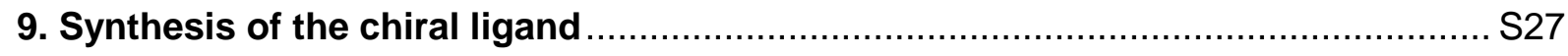

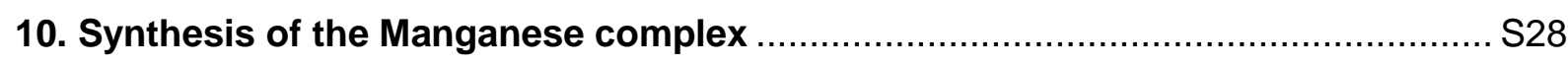

11. Analytical data of the observed homo-coupling products of the silyl enol ethers S29

12. NMR spectra of the synthesized compounds S34

13. References S60 


\section{General information}

All solvents were commercially available and have been distilled under reduced pressure prior to use. Solvents were dried over $3 \AA$ molecular sieve.

All chemicals or reagents were purchased from commercial suppliers without further purification, if not otherwise stated, or were prepared according to known literature procedures. If water or air sensitive compounds have been used, the experiments were carried out in heat gun dried glassware using conventional Schlenk techniques under nitrogen atmosphere. Electrochemical reactions were carried out using a TENMA potentiostat (72-10480, DigitaControl, DC Power Supply, 0-30 V, 3 A). All known compounds were characterized by ${ }^{1} \mathrm{H}$ and ${ }^{13} \mathrm{C}$ NMR. All unknown compounds were identified by ${ }^{1} \mathrm{H}$ NMR, ${ }^{13} \mathrm{C}$ NMR, IR and HRMS.

NMR spectroscopy: NMR spectra were recorded either on a Bruker Avance $300(300 \mathrm{MHz})$, on a Bruker Avance III (500 MHz) or on a Bruker Avance DRX (500 MHz). Chemical shifts are reported in parts per million (ppm). The spectra are referenced to the solvent peak of $\mathrm{CDCl}_{3}$. In the ${ }^{1} \mathrm{H}$ NMR spectra this corresponds with the singlet of the solvent signal of $\mathrm{CDCl}_{3}$ at $\delta=7.26 \mathrm{ppm}$. The ${ }^{13} \mathrm{C}$ NMR spectra were referenced to the central line of the triplet of $\mathrm{CDCl}_{3}$ at $\delta=77.16 \mathrm{ppm} .{ }^{[1]}$ The stated form of the signal describes the appearance of the signal and not the theoretically expected form.

Infrared Spectroscopy: The IR spectra were obtained by a Bruker Trensor 27. The wave numbers $\lambda^{-1}$ are quoted in reciprocal centimeters $\left(\mathrm{cm}^{-1}\right)$.

Chromatography: Flash chromatography was carried out using Machery-Nagel silica gel 60 (0.040-0.063 mm). Thin layer chromatography was carried out on Merck TLC plates coated with silica gel $60 \mathrm{~F}_{254}$ with fluorescence indicator. For the detection of the signals ultraviolet light $(\lambda=254 \mathrm{~nm})$ was used or heating after the plate has been dipped into a cerium(IV)molybdic-solution $\left(2 \mathrm{~g} \mathrm{Ce}\left(\mathrm{SO}_{4}\right)_{2}, 50 \mathrm{~g}\left(\mathrm{NH}_{4}\right)_{6} \mathrm{Mo}_{7} \mathrm{O}_{24} \cdot 4 \mathrm{H}_{2} \mathrm{O}, 50 \mathrm{~mL} \mathrm{H}_{2} \mathrm{SO}_{4}, 400 \mathrm{~mL} \mathrm{H} \mathrm{H}_{2}\right.$ ).

Gaschromatography (GC and GC/MS): GC analysis was performed on a Shimadzu GC2010 Plus series gas chromatograph with flame ionization detector (FID) and a Machery Nagel Optima 5 HT column (30 m). GC/MS spectra were recorded on a Shimadzu GC-2010 Plus gas chromatograph, which has a Machery Nagel Optima 5 HT column (30 m, 0.25 mm ID, $0.25 \mu \mathrm{m}$ film) and a Shimadzu GC/MS-QP2020 mass selective detector. Helium was used as carrier gas.

Polarimetry: The specific rotation $[\alpha]^{20} \mathrm{D}$ was determined by the Perkin Elmer polarimeter 343 with the utilization of the Na-D-line $(589 \mathrm{~nm})$ at $20^{\circ} \mathrm{C}$. A cuvette $(I=1 \mathrm{dm})$ was used. 
Cyclic voltammetry: Cyclic voltammetry measurements were carried out on a BAS C3 cell stand and a BAS 100 electrochemical analyser using a glassy carbon disk working electrode (2.0 $\mathrm{mm}$ diameter) and platinum wire counter electrode $(0.5 \mathrm{~mm}$ diameter). Potentials were referred to a saturated $\mathrm{Ag} / \mathrm{AgCl}(3 \mathrm{~mol} / \mathrm{L} \mathrm{NaCl})$ reference electrode. Before each experiment the solutions were purged with nitrogen.

\section{General procedures}

2.1. Preparation of the silyl enol ethers

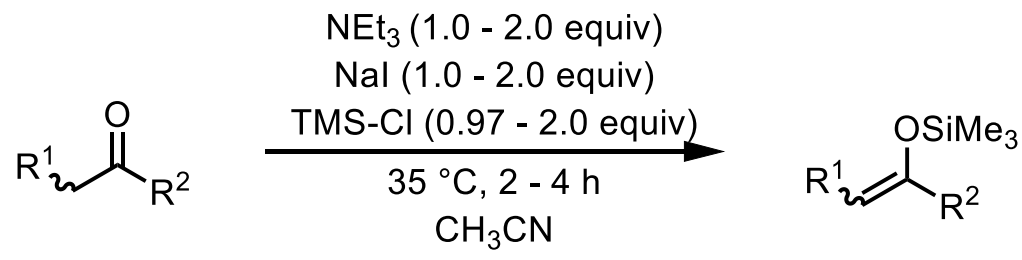

\section{General procedure A:}

According to literature procedures of WOODWARD ${ }^{[2]}$ and DUNOGUES ${ }^{[3]}$ the ketone (1.0 - 1.4 equiv) and triethylamine (1.0 - 2.0 equiv) were put into a three-necked-flask with stirring bar, internal thermometer and inert gas supply and chlorotrimethylsilane $(1.0-2.0$ equiv) was added over a period of 5 minutes. Then, the reaction mixture was heated to $35{ }^{\circ} \mathrm{C}$ and the oil bath was removed. A solution of sodium iodide $(1.0-2.0$ equiv) in anhydrous acetonitrile $(1.0 \mathrm{~mL} / \mathrm{mmol})$ was added in a way, that the temperature of the mixture stays between $34-40^{\circ} \mathrm{C}$. After stirring of the solution for $2-4 \mathrm{~h}$, the mixture was poured into ice water. The phases were separated and the aqueous phase was extracted with $n$-pentane. The combined organic layers were dried over magnesium sulfate and filtered. The silylenolethers were received after distillation as colorless liquids.

2.2. Electrochemical preparation of the diketoesters

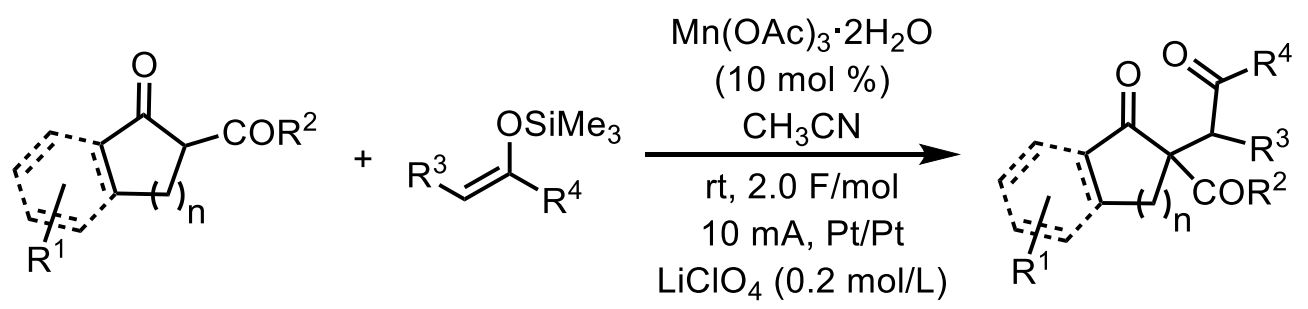

\section{General procedure B:}

At first $\mathrm{LiClO}_{4}(\mathrm{c}=0.2 \mathrm{~mol} / \mathrm{L}, 85.1 \mathrm{mg})$ and $\mathrm{Mn}(\mathrm{OAc})_{3} \cdot 2 \mathrm{H}_{2} \mathrm{O}(10 \mathrm{~mol} \%)$ were weight in a small vial $(10 \mathrm{~mL})$, which was equipped with a stirring bar, septum, intert gas supply and electrodes. A platinum sheet $\left(4 \mathrm{~cm}^{2}\right)$ was used as anode and a platinum wire as cathode. Afterwards, the reaction tube was evacuated twice and refilled with inert gas. Acetonitrile $(4.0 \mathrm{~mL})$, the 1,3dicarbonyl compound ( 0.25 or $0.50 \mathrm{mmol}, 1.0$ equiv) and the silyl enol ether (1.25 or 
$2.50 \mathrm{mmol}$, 5.0 equiv) were added through the septum. Then, the reaction mixture was electrolyzed under constant current $(10 \mathrm{~mA}, 1.0-2.0 \mathrm{~F} / \mathrm{mol})$. The diketones were obtained after flash chromatography.

\section{Optimization of the reaction conditions}

Table S1: Optimization of the reaction of $\mathbf{1 a}$ with enol acetate $\mathbf{6 a}$.

\begin{tabular}{|c|c|c|c|c|}
\hline & $-\mathrm{CO}_{2} \mathrm{Et}+5.0 \mathrm{ec}$ & $\begin{array}{c}\begin{array}{c}\mathrm{CeCl}_{3} \cdot 7 \mathrm{H}_{2} \mathrm{O} \\
\text { solvent, } \mathrm{rt}\end{array} \\
\underset{\mathrm{Pt} / \mathrm{Pt}, 10 \mathrm{~mA}}{\longrightarrow} \\
\mathrm{LiClO}_{4}(0.2 \mathrm{~mol} / \mathrm{L})\end{array}$ & ) & $y^{M e}$ \\
\hline no. & $\begin{array}{c}\text { catalyst loading } \\
(\mathrm{mol} \%)\end{array}$ & solvent & $\mathrm{F} / \mathrm{mol}$ & Yield (\%) \\
\hline 1 & 5 & TFE & 3.0 & traces \\
\hline 2 & 5 & TFE: $\mathrm{H}_{2} \mathrm{O}=7: 1$ & 3.0 & traces \\
\hline $3^{(\mathrm{a})}$ & 5 & TFE: $\mathrm{H}_{2} \mathrm{O}=9: 1$ & 3.0 & --- \\
\hline 4 & 5 & $\mathrm{MeOH}$ & 5.0 & --- \\
\hline 5 & 5 & $\mathrm{CH}_{3} \mathrm{CN}: \mathrm{H}_{2} \mathrm{O}=7: 1$ & 5.0 & 6 \\
\hline $6^{(\mathrm{b})}$ & 5 & $\mathrm{CH}_{3} \mathrm{CN}: \mathrm{H}_{2} \mathrm{O}=7: 1$ & 5.0 & traces \\
\hline 7 & 10 & $\mathrm{TFE}+2 \mathrm{vol} \% \mathrm{H}_{2} \mathrm{O}$ & 4.0 & $13^{(\mathrm{c})}$ \\
\hline 8 & 10 & $\mathrm{CH}_{3} \mathrm{CN}$ & 2.0 & $18^{(\mathrm{c})}$ \\
\hline 9 & --- & $\mathrm{CH}_{3} \mathrm{CN}$ & 2.0 & $9^{(c)}$ \\
\hline 10 & 10 & $\mathrm{CH}_{3} \mathrm{CN}+5$ vol $\% \mathrm{AcOH}$ & 2.0 & $33^{(\mathrm{c})}$ \\
\hline
\end{tabular}

Reaction conditions: The reactions were performed under inert atmosphere utilizing a platinum plate electrode as anode and a platinum wire cathode. (a) Divided cell. $\mathrm{c}\left(\mathrm{LiClO}_{4}\right)=0.3 \mathrm{~mol} / \mathrm{L}$, (b) $\mathrm{TBABF}_{4}$ as supporting electrolyte, (c) The yield was determined by GC analysis utilizing mesitylene as internal standard. TFE $=2,2,2$-trifluoroethanol. 
Table S2: Influence of different metal salt additives on the reaction of 1a with enol acetate $6 a$.

\begin{tabular}{|c|c|c|c|}
\hline $1 \mathrm{a}$ & $5.0 \mathrm{eq} \int_{\mathbf{6 a}}^{\mathrm{OAc}}$ & $\begin{array}{c}\text { catalyst }(10 \mathrm{~mol} \%) \\
\mathrm{rt} \\
\mathrm{Pt} / \mathrm{Pt}, 10 \mathrm{~mA} \\
\mathrm{LiClO}_{3}(0.2 \mathrm{~mol} / \mathrm{L})\end{array}$ & o \\
\hline no. & metal salt & F/mol & Yield (\%) \\
\hline 1 & $\mathrm{CeCl}_{3} \cdot 7 \mathrm{H}_{2} \mathrm{O}$ & 2.0 & 33 \\
\hline 2 & $\mathrm{Co}(\mathrm{OAc})_{2} \cdot 4 \mathrm{H}_{2} \mathrm{O}$ & 1.5 & 24 \\
\hline 3 & $\left(\mathrm{NH}_{4}\right)_{2} \mathrm{Ce}\left(\mathrm{NO}_{2}\right)_{6}$ & 1.5 & 32 \\
\hline 4 & $\mathrm{CuCl}_{2} \cdot 2 \mathrm{H}_{2} \mathrm{O}$ & 3.0 & 23 \\
\hline 5 & $\mathrm{MnCl}_{2} \cdot 4 \mathrm{H}_{2} \mathrm{O}$ & 2.0 & 18 \\
\hline 6 & $\mathrm{Mn}(\mathrm{OAc})_{3} \cdot 2 \mathrm{H}_{2} \mathrm{O}$ & 2.0 & 37 \\
\hline
\end{tabular}

Reaction conditions: The reactions were performed under inert atmosphere utilizing a platinum plate electrode as anode and a platinum wire cathode. (a) The yield was determined by GC analysis utilizing mesitylene as internal standard.

Table S3: Optimization of the amount of acetic acid.

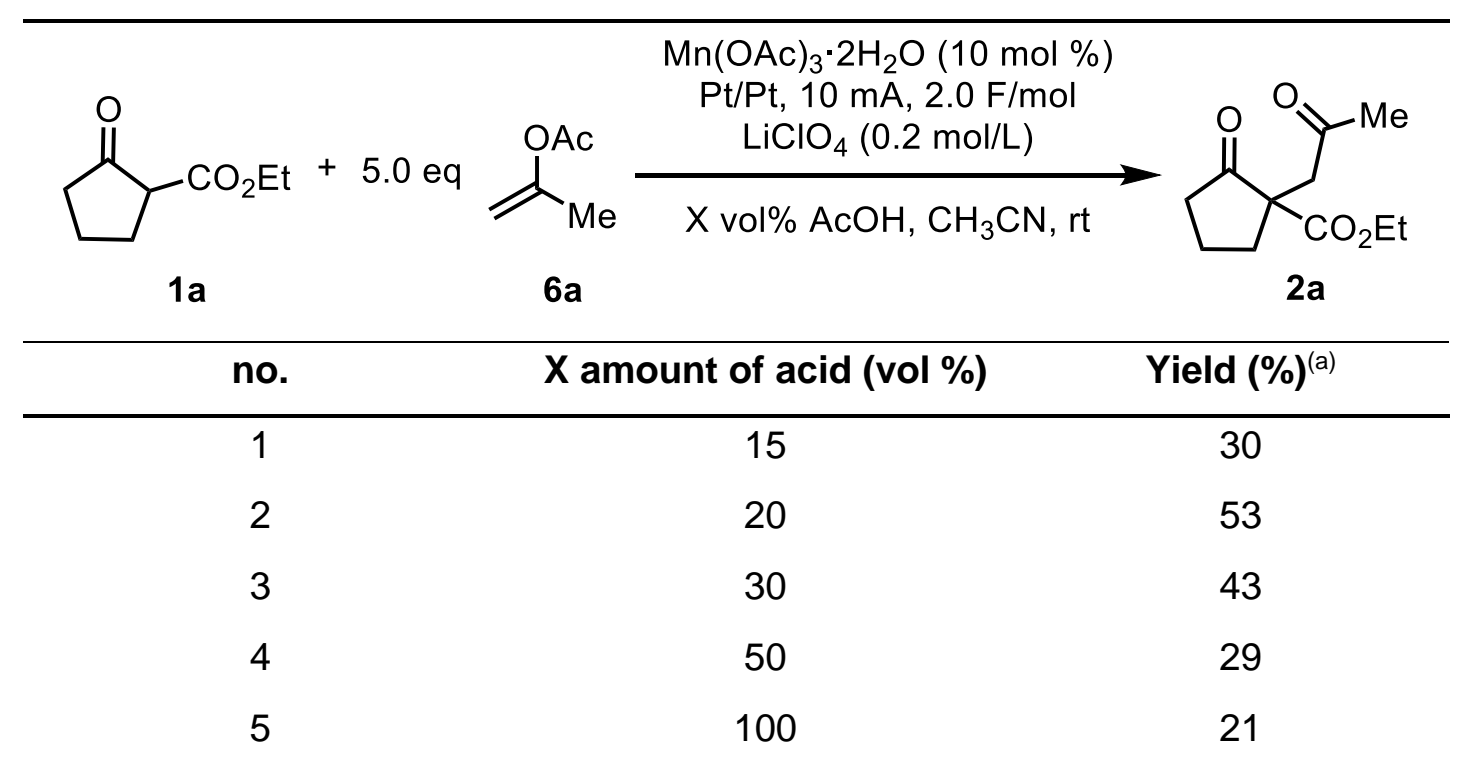

Reaction conditions: The reactions were performed under inert atmosphere utilizing a platinum plate electrode as anode and a platinum wire cathode. (a) The yield was determined by GC analysis utilizing mesitylene as internal standard. 
Table S4: Optimization of the catalyst on the reaction of $1 \mathbf{a}$ with silyl enol ether $\mathbf{6 b}$.

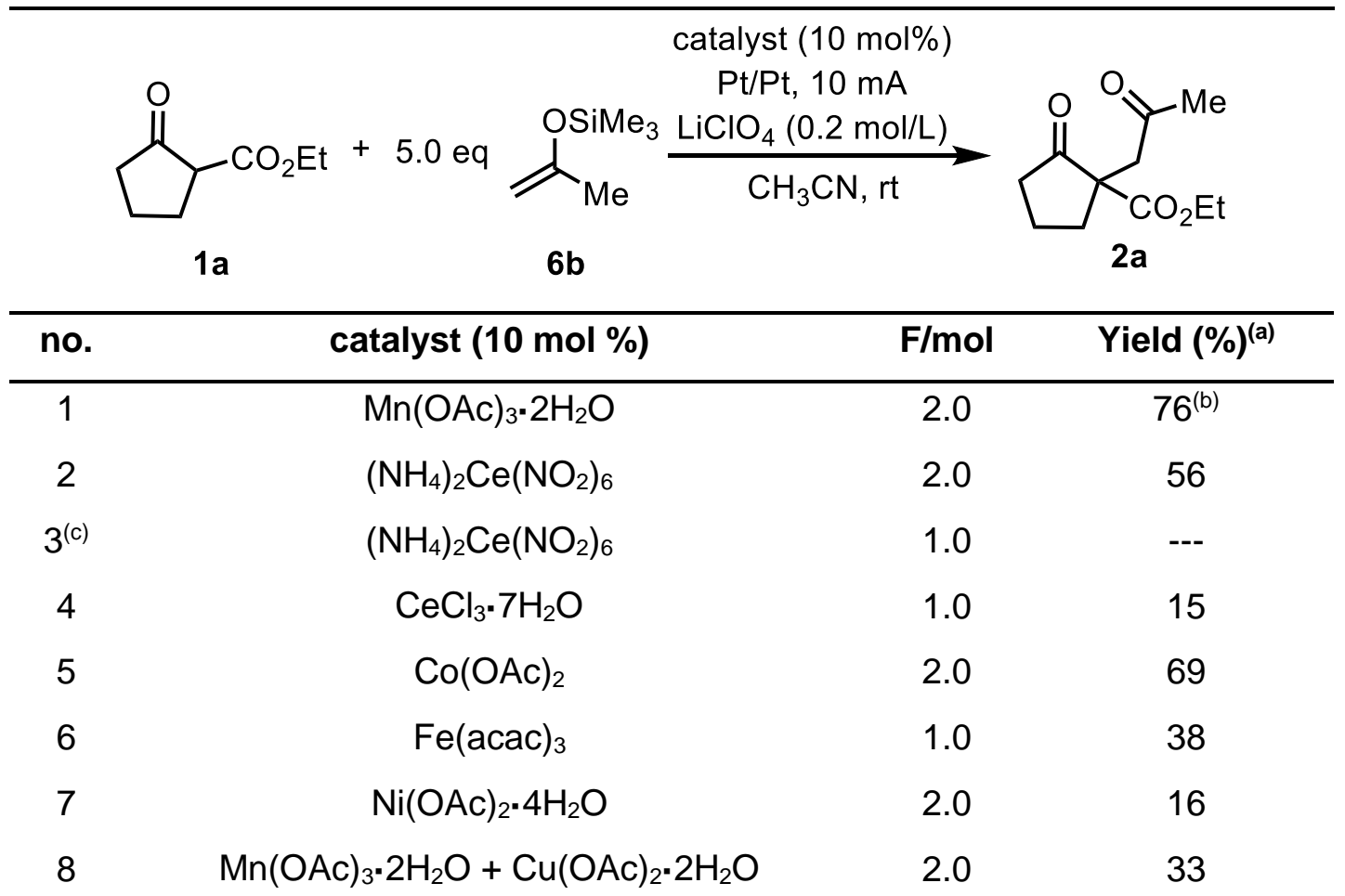

Reaction conditions: The reactions were performed under inert atmosphere utilizing a platinum plate electrode as anode and a platinum wire cathode. (a) The yield was determined by GC analysis utilizing mesitylene as internal standard. (b) Isolated yield. (c) Addition of acetic acid (5 vol \%). 
Table S5: Optimization of additional reaction parameters.

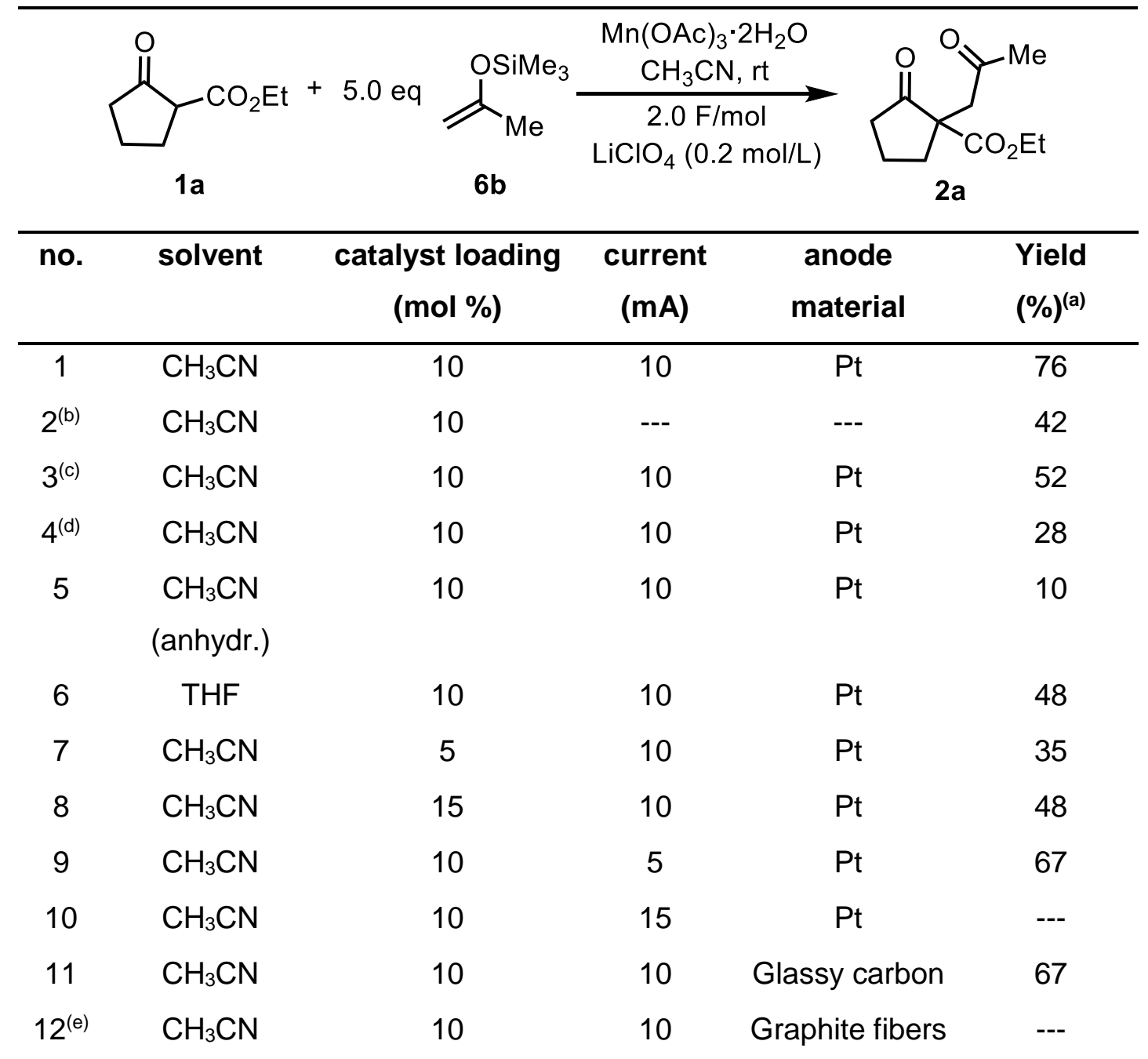

Reaction conditions: The reactions were performed under inert atmosphere utilizing a platinum plate electrode as anode and a platinum wire cathode. (a) The yield was determined by GC analysis utilizing mesitylene as internal standard. (b) Oxygen was used as oxidizing agent for 16 h. (d) 2.0 Equivalent of 6 b were used. (e) Divided cell. 


\section{Cyclovoltammograms}

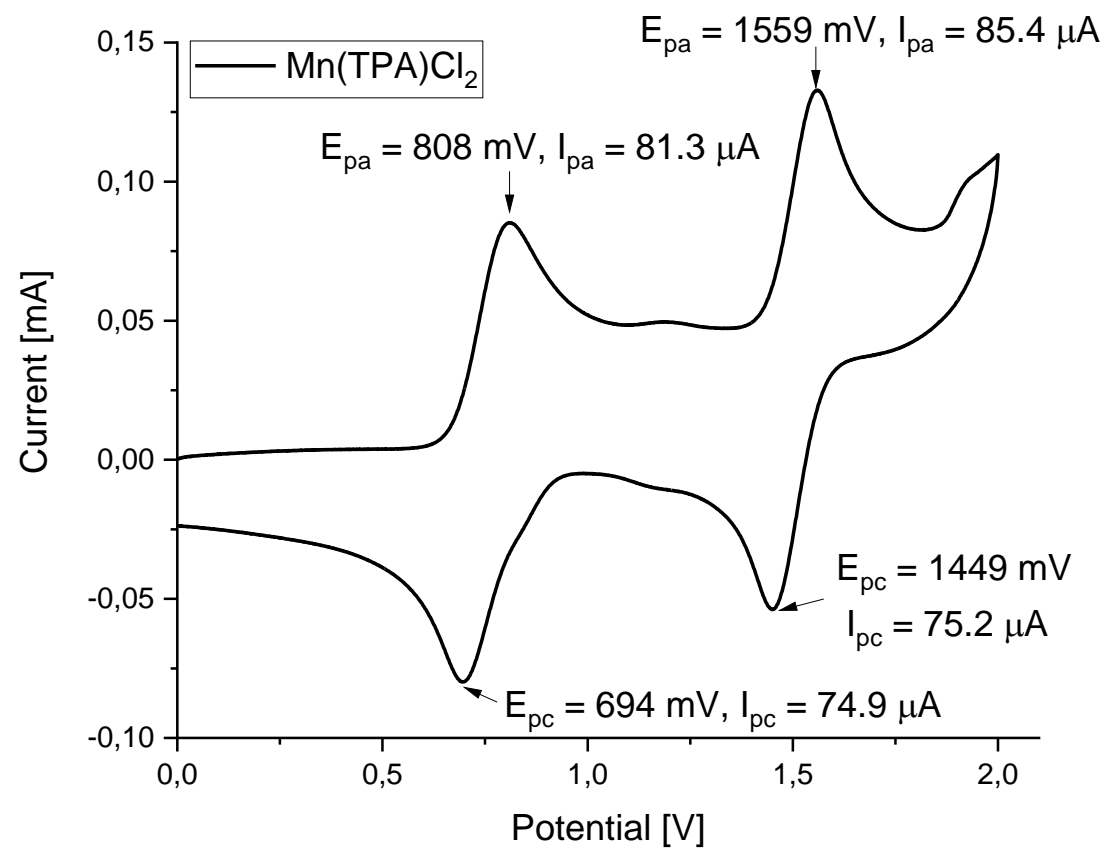

Figure 1: Cyclic voltammogram of $\mathrm{Mn}(\mathrm{TPA}) \mathrm{Cl}_{2}(3 \mathrm{mmol} / \mathrm{L})$ in $\mathrm{CH}_{3} \mathrm{CN} / \mathrm{LiClO}_{4}(10 \mathrm{~mL}, 0.1 \mathrm{~mol} / \mathrm{L})$. Reference electrode: $\mathrm{Ag} / \mathrm{AgCl}(3 \mathrm{~mol} / \mathrm{L} \mathrm{NaCl})$, scan rate $=250 \mathrm{mVs}^{-1}$.

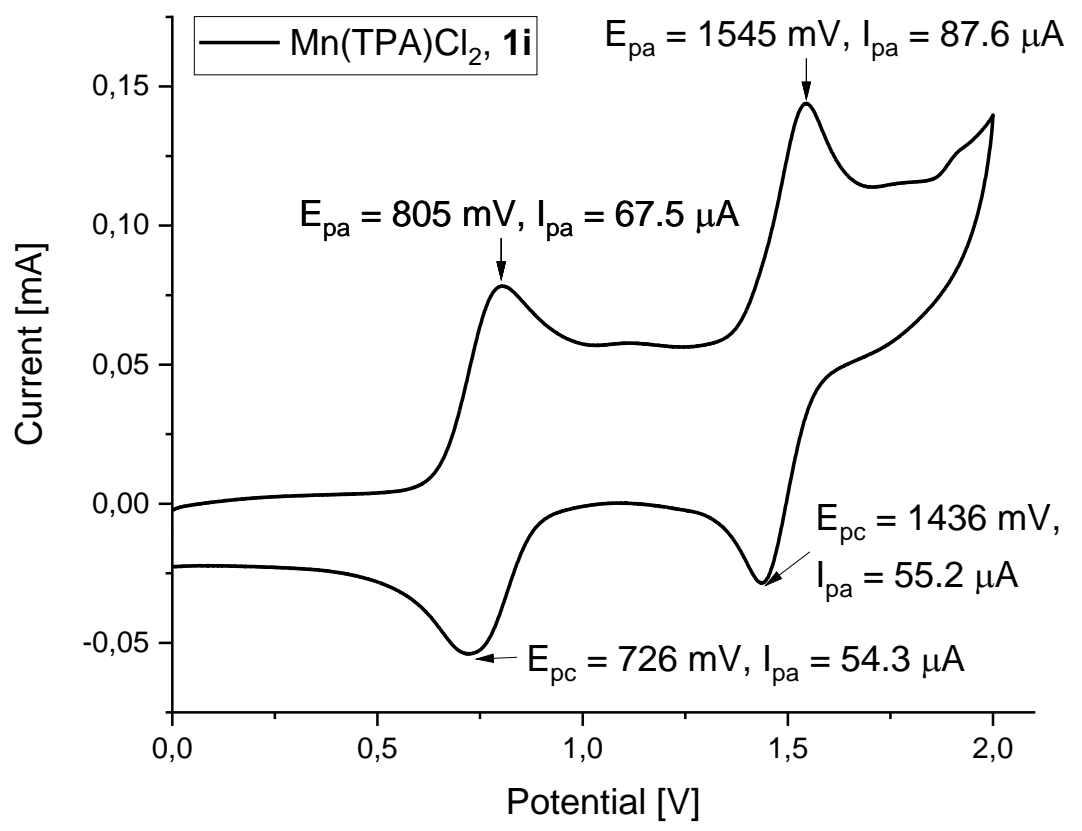

Figure 2: Cyclic voltammogram of $\mathrm{Mn}(\mathrm{TPA}) \mathrm{Cl}_{2}(3 \mathrm{mmol} / \mathrm{L})$ and $1 \mathrm{i}$ (3.0 equiv) in $\mathrm{CH}_{3} \mathrm{CN} / \mathrm{LiClO}_{4}(10 \mathrm{~mL}, 0.1 \mathrm{~mol} / \mathrm{L})$. Reference electrode: $\mathrm{Ag} / \mathrm{AgCl}(3 \mathrm{~mol} / \mathrm{L} \mathrm{NaCl})$, scan rate $=250 \mathrm{mVs}^{-1}$. 


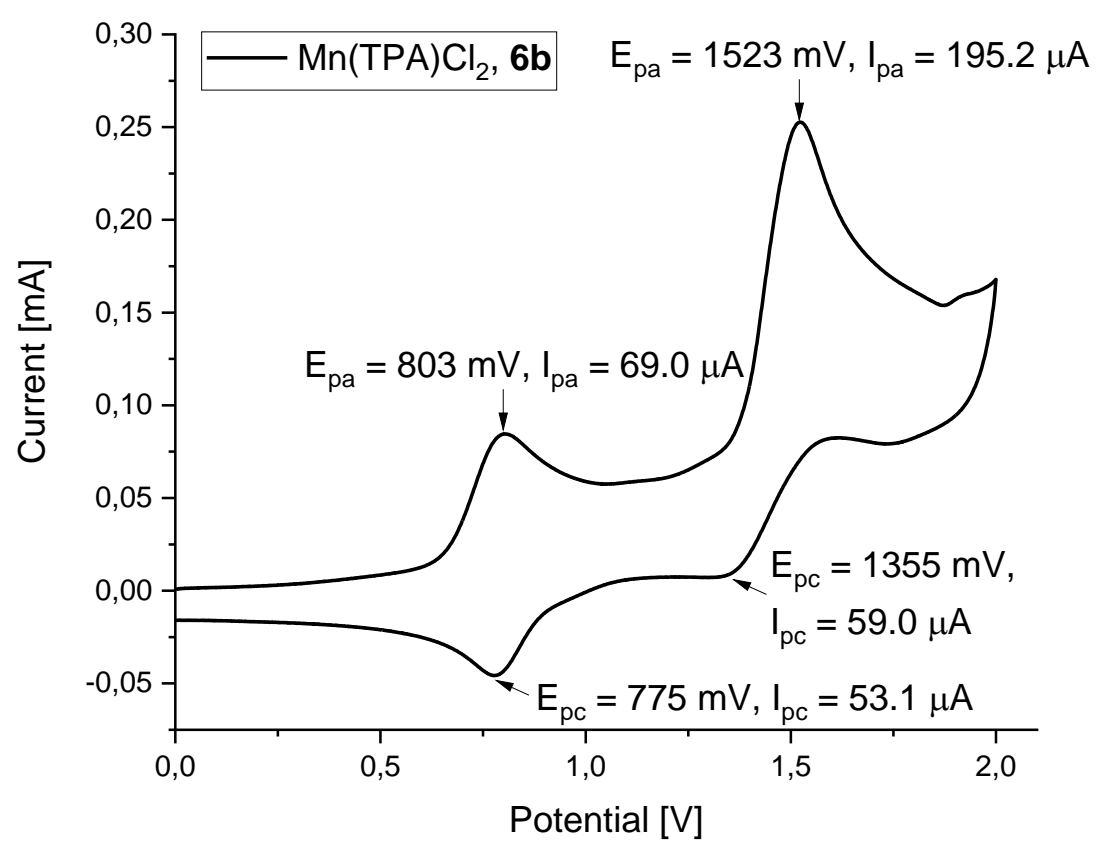

Figure 3: Cyclic voltammogram of $\mathrm{Mn}(\mathrm{TPA}) \mathrm{Cl}_{2}(3 \mathrm{mmol} / \mathrm{L})$ and $6 \mathbf{b}\left(9.0\right.$ equiv) in $\mathrm{CH}_{3} \mathrm{CN} / \mathrm{LiClO}_{4}(10 \mathrm{~mL}, 0.1 \mathrm{~mol} / \mathrm{L})$. Reference electrode: $\mathrm{Ag} / \mathrm{AgCl}(3 \mathrm{~mol} / \mathrm{L} \mathrm{NaCl})$, scan rate $=250 \mathrm{mVs}^{-1}$.

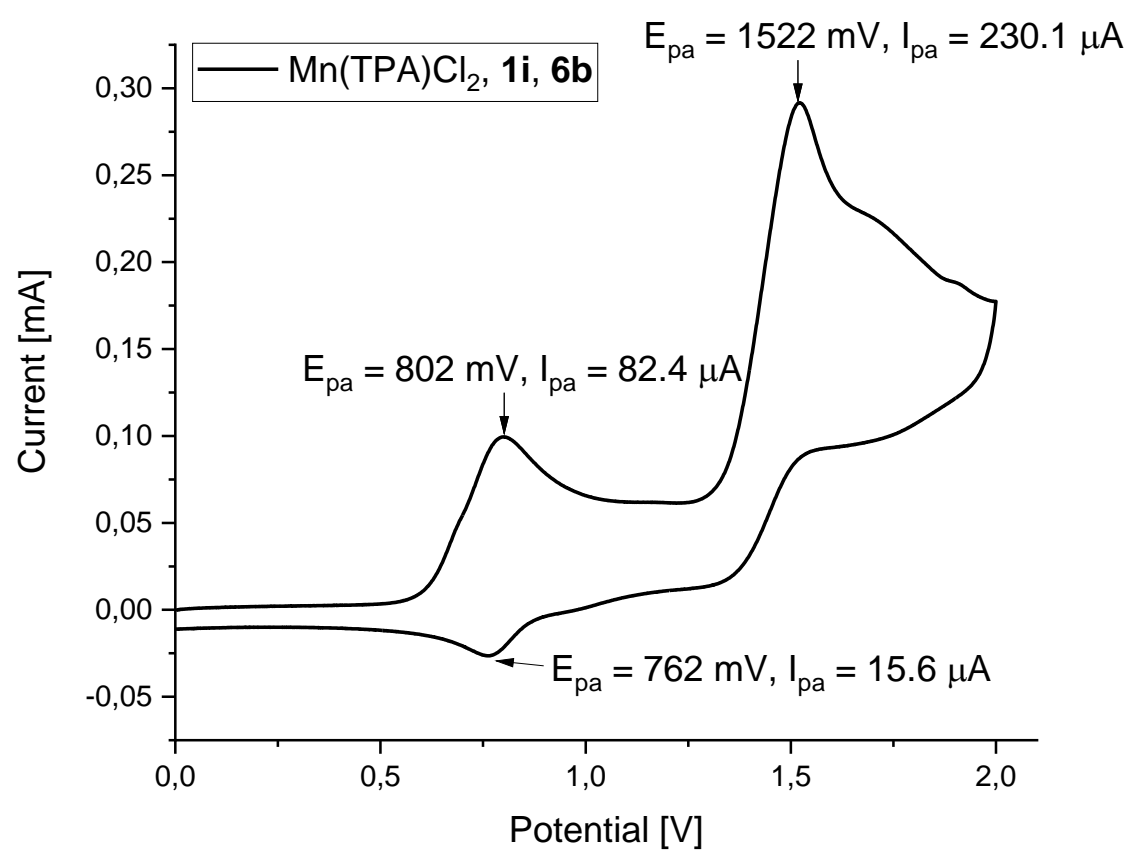

Figure 4: Cyclic voltammogram of $\mathrm{Mn}(\mathrm{TPA}) \mathrm{Cl}_{2}$ (3 mmol/L), $\mathbf{1 i}\left(3.0\right.$ equiv) and $\mathbf{6 b}$ (9.0 equiv) in $\mathrm{CH}_{3} \mathrm{CN}_{2} / \mathrm{LiClO}_{4}(10$ $\mathrm{mL}, 0.1 \mathrm{~mol} / \mathrm{L})$. Reference electrode: $\mathrm{Ag} / \mathrm{AgCl}(3 \mathrm{~mol} / \mathrm{L} \mathrm{NaCl})$, scan rate $=250 \mathrm{mVs}^{-1}$. 


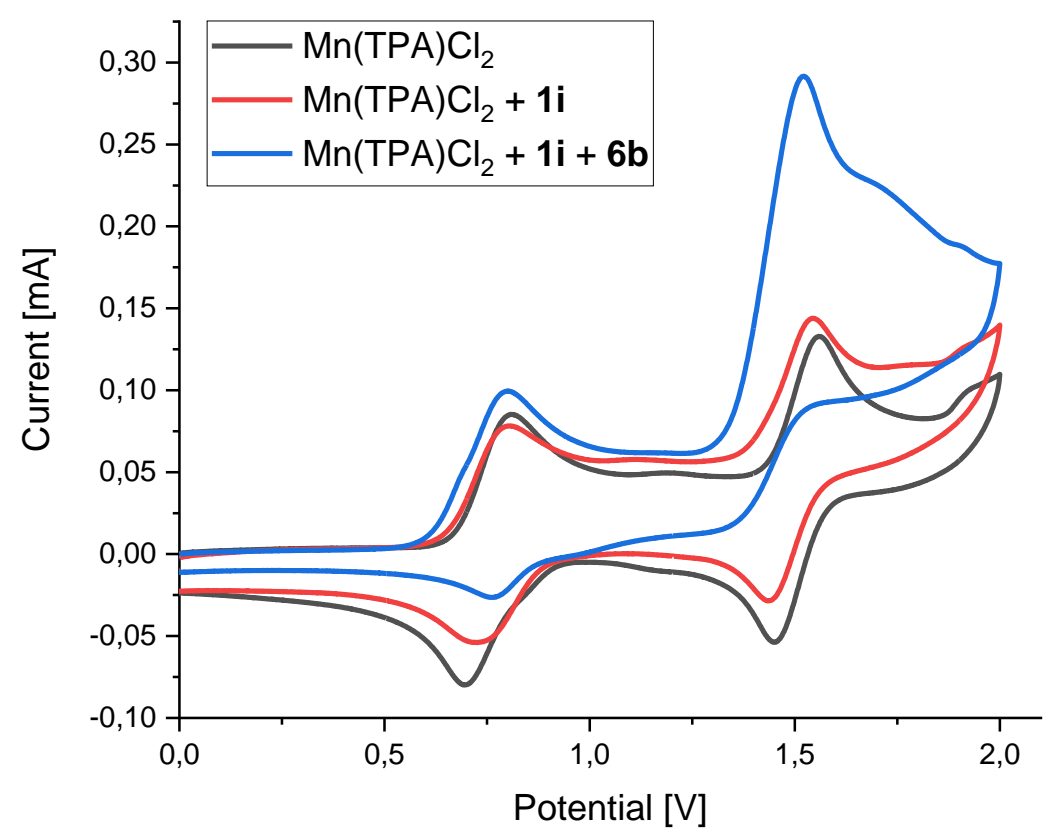

Figure 5: Cyclic voltammogram of: black: $\mathrm{Mn}(\mathrm{TPA}) \mathrm{Cl}_{2}(3 \mathrm{mmol} / \mathrm{L})$; red: $\mathrm{Mn}(\mathrm{TPA}) \mathrm{Cl}_{2}(3 \mathrm{mmol} / \mathrm{L})$ and 1i (3.0 equiv); blue: $\mathrm{Mn}(\mathrm{TPA}) \mathrm{Cl}_{2}(3 \mathrm{mmol} / \mathrm{L}), \mathbf{1 i}$ (3.0 equiv) and $\mathbf{6 b}$ (9.0 equiv) in $\mathrm{CH}_{3} \mathrm{CN} / \mathrm{LiClO}_{4}(10 \mathrm{~mL}, 0.1 \mathrm{~mol} / \mathrm{L})$. Reference electrode: $\mathrm{Ag} / \mathrm{AgCl}(3 \mathrm{~mol} / \mathrm{L} \mathrm{NaCl})$, scan rate $=250 \mathrm{mVs}^{-1}$.

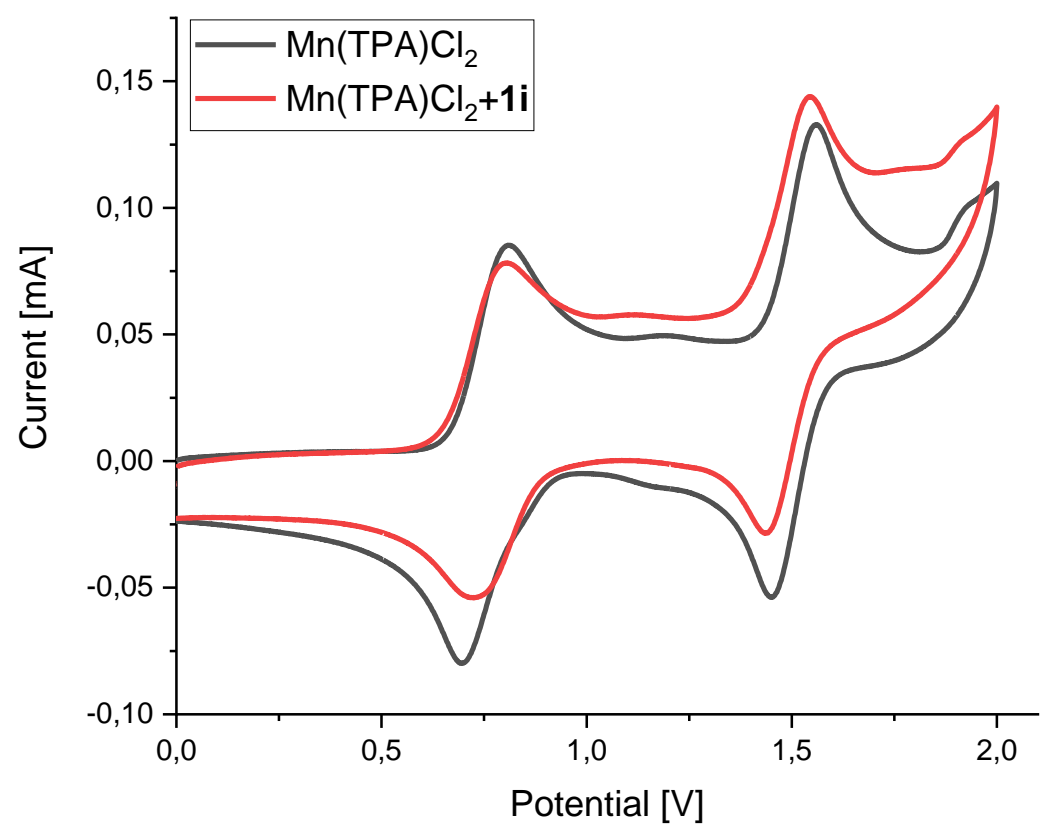

Figure 6: Cyclic voltammogram of: black: $\mathrm{Mn}(\mathrm{TPA}) \mathrm{Cl}_{2}(3 \mathrm{mmol} / \mathrm{L})$; red: $\mathrm{Mn}(\mathrm{TPA}) \mathrm{Cl}_{2}(3 \mathrm{mmol} / \mathrm{L})$ and $1 \mathbf{i}$ (3.0 equiv) in $\mathrm{CH}_{3} \mathrm{CN} / \mathrm{LiClO}_{4}(10 \mathrm{~mL}, 0.1 \mathrm{~mol} / \mathrm{L})$. Reference electrode: $\mathrm{Ag} / \mathrm{AgCl}(3 \mathrm{~mol} / \mathrm{L} \mathrm{NaCl})$, scan rate $=250 \mathrm{mVs}^{-1}$. 


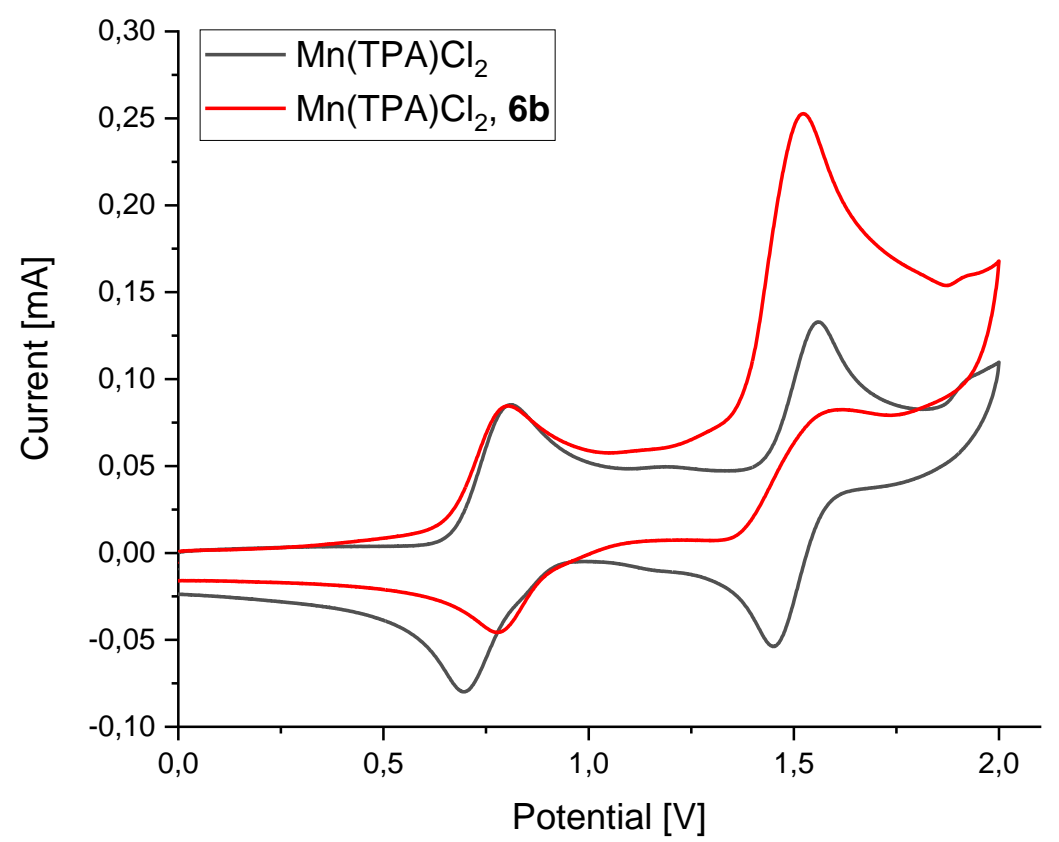

Figure 7: Cyclic voltammogram of: black: $\mathrm{Mn}(\mathrm{TPA}) \mathrm{Cl}_{2}(3 \mathrm{mmol} / \mathrm{L})$; red: $\mathrm{Mn}(\mathrm{TPA}) \mathrm{Cl}_{2}(3 \mathrm{mmol} / \mathrm{L})$ and $6 \mathbf{b}$ (9.0 equiv) in $\mathrm{CH}_{3} \mathrm{CN} / \mathrm{LiClO}_{4}(10 \mathrm{~mL}, 0.1 \mathrm{~mol} / \mathrm{L})$. Reference electrode: $\mathrm{Ag} / \mathrm{AgCl}(3 \mathrm{~mol} / \mathrm{L} \mathrm{NaCl})$, scan rate $=250 \mathrm{mVs}^{-1}$.

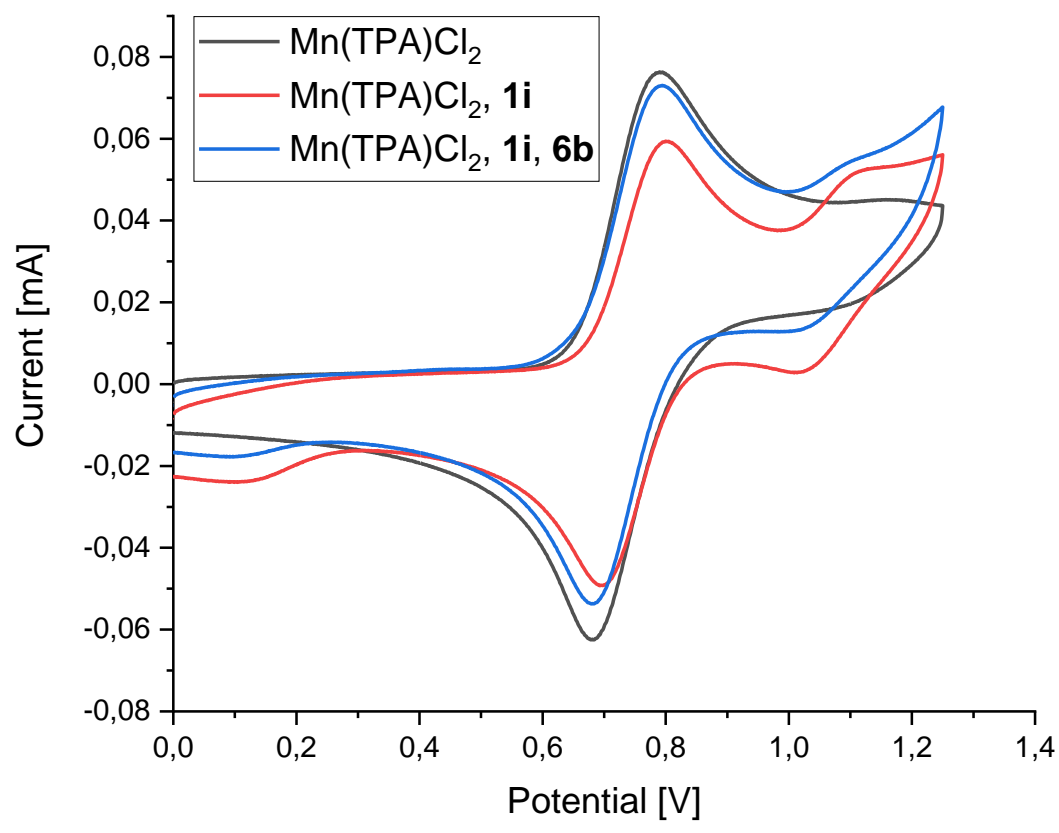

Figure 8: Cyclic voltammogram of: black: $\mathrm{Mn}(\mathrm{TPA}) \mathrm{Cl}_{2}$ (3 mmol/L); red: $\mathrm{Mn}(\mathrm{TPA}) \mathrm{Cl}_{2}(3 \mathrm{mmol} / \mathrm{L})$ and 1i (3.0 equiv); blue: $\mathrm{Mn}(\mathrm{TPA}) \mathrm{Cl}_{2}$ (3 mmol/L), 1i (3.0 equiv) and $\mathbf{6 b}$ (9.0 equiv) in $\mathrm{CH}_{3} \mathrm{CN} / \mathrm{LiClO}_{4}(10 \mathrm{~mL}, 0.1 \mathrm{~mol} / \mathrm{L})$. Reference electrode: $\mathrm{Ag} / \mathrm{AgCl}(3 \mathrm{~mol} / \mathrm{L} \mathrm{NaCl})$, scan rate $=250 \mathrm{mVs}^{-1}$. 


\section{Synthesis of the silyl enol ethers}

\section{2-(trimethylsiloxy)propene}<smiles>C=C(C)OCC</smiles>

According to the general procedure A the product was synthesized from acetone $(6.29 \mathrm{~g}$, $108 \mathrm{mmol}, 1.4$ equiv), triethylamine ( $7.82 \mathrm{~g}, 77.3 \mathrm{mmol}, 1.0$ equiv), sodium iodide ( $11.59 \mathrm{~g}$, $77.30 \mathrm{mmol}$, 1.0 equiv), and chlorotrimethylsilane $(8.15 \mathrm{~g}, 75.0 \mathrm{mmol}, 0.97$ equiv). After stirring for $2 \mathrm{~h}$ and aqueous work-up the product $(6.85 \mathrm{~g}, 52.6 \mathrm{mmol}, 70 \%)$ was obtained as a colorless liquid after fractional distillation (head temperature: $95^{\circ} \mathrm{C}$ ) under inert gas.

${ }^{1} \mathrm{H}$ NMR $\left(300 \mathrm{MHz}, \mathrm{CDCl}_{3}\right): \delta=4.05(\mathrm{~s}, 2 \mathrm{H}), 1.78(\mathrm{~s}, 3 \mathrm{H}), 0.21(\mathrm{~s}, 9 \mathrm{H})$ ppm. ${ }^{13} \mathrm{C}$ NMR $\left(125 \mathrm{MHz}, \mathrm{CDCl}_{3}\right): \delta=156.2,91.4,23.0,0.3 \mathrm{ppm}$.

The data were in accordance with literature values. ${ }^{[3]}$

\section{3,3-dimethyl-2-trimethylsiloxy-1-butene}<smiles>C=C(O[SiH3])C(C)(C)C</smiles>

According to the general procedure A the product was synthesized from 3,3-dimethylbutanone ( $1.20 \mathrm{~g}, 12.0 \mathrm{mmol}, 1.0$ equiv), triethylamine (1.46 g, $14.4 \mathrm{mmol}, 1.2$ equiv), sodium iodide ( $2.16 \mathrm{~g}, 14.4 \mathrm{mmol}, 1.2$ equiv), and chlorotrimethylsilane (1.56 g, $14.4 \mathrm{mmol}, 1.2$ equiv). After stirring for $4 \mathrm{~h}$ and aqueous work-up the product (936 $\mathrm{mg}, 5.43 \mathrm{mmol}, 45 \%$ ) was obtained as a colorless liquid after removal of the solvent and impurities via distillation.

${ }^{1} \mathrm{H}$ NMR $\left(500 \mathrm{MHz}, \mathrm{CDCl}_{3}\right): \delta=4.09-4.08(\mathrm{~m}, 1 \mathrm{H}), 3.93-3.92(\mathrm{~m}, 1 \mathrm{H}), 1.05(\mathrm{~s}, 9 \mathrm{H}), 0.21(\mathrm{~s}$, $9 \mathrm{H}) \mathrm{ppm} .{ }^{13} \mathrm{C}$ NMR $\left(125 \mathrm{MHz}, \mathrm{CDCl}_{3}\right): \delta=167.4,85.9,36.6,28.2,0.3 \mathrm{ppm}$.

The data were in accordance with literature values. ${ }^{[4]}$ 


\section{1-trimethylsiloxycyclohexene}

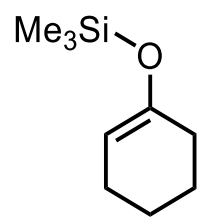

According to the general procedure $A$ the product was synthesized from cyclohexanone $(1.18 \mathrm{~g}, 12.0 \mathrm{mmol}, 1.0 \mathrm{eq})$, triethylamine $(1.46 \mathrm{~g}, 14.4 \mathrm{mmol}, 1.2$ equiv), sodium iodide $(2.16 \mathrm{~g}, 14.4 \mathrm{mmol}, 1.2 \mathrm{eq})$, and chlorotrimethylsilane (1.56 g, $14.4 \mathrm{mmol}, 1.2$ equiv). After stirring for $3 \mathrm{~h}$ and aqueous work-up the product $(1.15 \mathrm{~g}, 6.75 \mathrm{mmol}, 56 \%)$ was obtained as a colorless liquid after fractional distillation under reduced pressure with Vigreux column (35 mbar, head temperature: $70^{\circ} \mathrm{C}$ ).

${ }^{1} \mathrm{H}$ NMR $\left(500 \mathrm{MHz}, \mathrm{CDCl}_{3}\right): \delta=4.89-4.83(\mathrm{~m}, 1 \mathrm{H}), 2.03-1.94(\mathrm{~m}, 4 \mathrm{H}), 1.68-1.62(\mathrm{~m}, 2 \mathrm{H})$, 1.54-1.47 (m, $2 \mathrm{H}), 0.18(\mathrm{~s}, 9 \mathrm{H})$ ppm. ${ }^{13} \mathrm{C}$ NMR (125 MHz, $\left.\mathrm{CDCl}_{3}\right): \delta=150.5,104.4,30.1$, 24.0, 23.4, 22.5, $0.5 \mathrm{ppm}$.

The data were in accordance with literature values. ${ }^{[5]}$

\section{2-trimethylsiloxy-1-heptene}

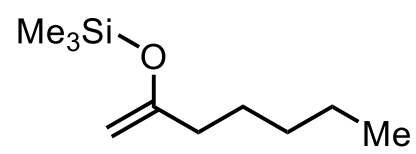

According to a reported procedure of NovÁk ${ }^{[6]}$ diisopropylamine $(1.46 \mathrm{~g}, 14.4 \mathrm{mmol}, 1.2$ equiv) was dissolved in abs. THF $(25 \mathrm{~mL})$ and cooled down to $-78^{\circ} \mathrm{C}$. At this temperature $n$-BuLi (5.00 mL, $12.7 \mathrm{mmol}, 1.1$ equiv, $2.5 \mathrm{~mol} / \mathrm{L}$ in hexane) was added. After stirring of the reaction mixture for 10 minutes, a solution of 2-heptanone $(1.37 \mathrm{~g}, 12.0 \mathrm{mmol}, 1.0$ equiv) in abs. THF $(2.5 \mathrm{~mL})$ was added dropwise at the same temperature. The reaction mixture was stirred for $1 \mathrm{~h}$ at $-78^{\circ} \mathrm{C}$ and then chlorotrimethylsilane $(2.22 \mathrm{~g}, 20.4 \mathrm{mmol}, 1.7$ equiv) was added fast. Afterwards, the solution was stirred for $1 \mathrm{~h}$ at room temperature. Then, it was filtered through a pad of celite and THF was removed under reduced pressure. The residue was dissolved in $n$-pentane $(15 \mathrm{~mL})$ and filtered over celite again. After removal of $n$-pentane under reduced pressure, the crude product was distilled under reduced pressure (70 mbar, head temperature: $\left.96^{\circ} \mathrm{C}\right)$. Thus, the product $(1.01 \mathrm{~g}, 5.42 \mathrm{mmol}, 45 \%)$ was isolated as a colorless liquid.

${ }^{1} \mathrm{H}$ NMR $\left(500 \mathrm{MHz}, \mathrm{CDCl}_{3}\right): \delta=4.04(\mathrm{~s}, 1 \mathrm{H}), 4.03(\mathrm{~s}, 1 \mathrm{H}), 2.00(\mathrm{t}, 2 \mathrm{H}, J=7.4 \mathrm{~Hz}), 1.35-1.24$ $(\mathrm{m}, 6 \mathrm{H}), 0.89(\mathrm{t}, 3 \mathrm{H}, J=7.0 \mathrm{~Hz}), 0.20(\mathrm{~s}, 9 \mathrm{H}) \mathrm{ppm} .{ }^{13} \mathrm{C}$ NMR $\left(125 \mathrm{MHz}, \mathrm{CDCl}_{3}\right): \delta=159.8$, 89.9, 36.6, 31.5, 26.7, 22.7, 14.2, 0.3 ppm. 
The data of the ${ }^{1} \mathrm{H}$ NMR were in accordance with literature values. ${ }^{[6]}$ The data for the ${ }^{13} \mathrm{C}$ NMR could not be found in the literature.

\section{a-trimethylsiloxystyrene}

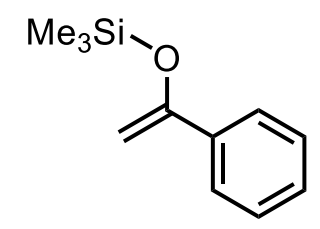

According to the general procedure $A$ the product was synthesized from acetophenone $(1.44 \mathrm{~g}$, $12.0 \mathrm{mmol}$, 1.0 equiv), triethylamine $(2.43 \mathrm{~g}, 24.0 \mathrm{mmol}, 2.0$ equiv), sodium iodide ( $3.59 \mathrm{~g}$, $24.0 \mathrm{mmol}, 2.0 \mathrm{eq})$, and chlorotrimethylsilane (2.61 g, $24.0 \mathrm{mmol}, 2.0$ equiv). After stirring for $2.5 \mathrm{~h}$ and aqueous work-up the product $(1.75 \mathrm{~g}, 9.10 \mathrm{mmol}, 76 \%)$ was obtained as a colorless liquid after fractional distillation under reduced pressure with Vigreux column (1.2 mbar, head temperature: $52^{\circ} \mathrm{C}$ ).

${ }^{1} \mathrm{H}$ NMR $\left(300 \mathrm{MHz}, \mathrm{CDCl}_{3}\right): \delta=7.62-7.56(\mathrm{~m}, 2 \mathrm{H}), 7.36-7.27(\mathrm{~m}, 3 \mathrm{H}), 4.92(\mathrm{~d}, 1 \mathrm{H}$, $J=1.4 \mathrm{~Hz}), 4.43(\mathrm{~d}, 1 \mathrm{H}, J=1.4 \mathrm{~Hz}), 0.27(\mathrm{~s}, 9 \mathrm{H}) \mathrm{ppm} .{ }^{13} \mathrm{C}$ NMR $\left(75 \mathrm{MHz}, \mathrm{CDCl}_{3}\right): \delta=155.8$, 137.6, 128.7, 128.2, 125.3, 91.2, 0.2 ppm.

The data were in accordance with literature values. ${ }^{[7]}$

\section{$\alpha$-trimethylsiloxy-4-nitrostyrene}<smiles>C=C(O[SiH3])c1ccc([N+](=O)[O-])cc1</smiles>

According to the general procedure $A$ the product was synthesized from 4-nitroacetophenone $(2.50 \mathrm{~g}, 15.0 \mathrm{mmol}, 1.0 \mathrm{eq})$, triethylamine $(1.67 \mathrm{~g}, 16.5 \mathrm{mmol}, 1.1$ equiv), sodium iodide $(2.47 \mathrm{~g}, 16.5 \mathrm{mmol}, 1.1 \mathrm{eq})$, and chlorotrimethylsilane $(1.79 \mathrm{~g}, 16.5 \mathrm{mmol}, 1.1$ equiv). After stirring for $3 \mathrm{~h}$ and aqueous work-up the product $(2.83 \mathrm{~g}, 11.9 \mathrm{mmol}, 79 \%)$ was obtained as a yellowish liquid after fractional distillation under vacuum $\left(1 \cdot 10^{-1} \mathrm{mbar}\right.$, head temperature: $\left.75^{\circ} \mathrm{C}\right)$.

${ }^{1} \mathrm{H}$ NMR $\left(300 \mathrm{MHz}, \mathrm{CDCl}_{3}\right): \delta=8.21-8.15(\mathrm{~m}, 2 \mathrm{H}), 7.77-7.70(\mathrm{~m}, 2 \mathrm{H}), 5.08(\mathrm{~d}, 1 \mathrm{H}$, $J=2.3 \mathrm{~Hz}), 4.62(\mathrm{~d}, 1 \mathrm{H}, J=2.3 \mathrm{~Hz}), 0.29(\mathrm{~s}, 9 \mathrm{H}) \mathrm{ppm} .{ }^{13} \mathrm{C} \mathrm{NMR}\left(75 \mathrm{MHz}, \mathrm{CDCl}_{3}\right): \delta=153.9$, 147.6, 143.8, 126.0, 123.6, 94.6, 0.2 ppm. GC/MS (EI): m/z (\%): 237 ([M+], 13), 237 (13), 236 
(9), 222 (47), 221 (19), 220 (100), 191 (29), 190 (53), 180 (20), 161 (18), 75 (65), 73 (53). HRMS (El): $\mathrm{m} / \mathrm{z}$ calculated for $\mathrm{C}_{14} \mathrm{H}_{15} \mathrm{NO}_{3} \mathrm{Si}: 237.0821$, found: 237.0825 .

The compound is literature known, but has not been characterized sufficiently. The ${ }^{1} \mathrm{H}$ NMR data are in accordance with literature values. ${ }^{[8]}$

\section{4-methoxy- $\alpha$-trimethylsiloxystyrene}

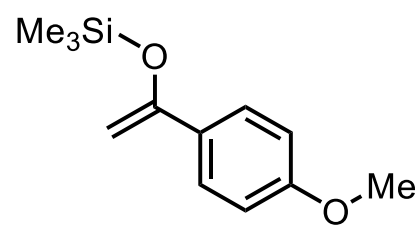

According to the general procedure $A$ the product was synthesized from 4-methoxyacetophenone ( $1.20 \mathrm{~g}, 7.99 \mathrm{mmol}, 1.0$ equiv), triethylamine $(1.60 \mathrm{~g}, 16.0 \mathrm{mmol}, 2.0$ equiv), sodium iodide (2.40 g, $16.0 \mathrm{mmol}, 2.0$ equiv), and chlorotrimethylsilane $(1.74 \mathrm{~g}, 16.0 \mathrm{mmol}$, 2.0 equiv). After stirring for $4 \mathrm{~h}$ and aqueous work-up the product ( $1.14 \mathrm{~g}, 5.12 \mathrm{mmol}, 64 \%$ ) was obtained as a colorless liquid after fractional distillation under vacuum $\left(5 \cdot 10^{-2} \mathrm{mbar}\right.$, head temperature: $\left.53^{\circ} \mathrm{C}\right)$.

${ }^{1} \mathrm{H}$ NMR $\left(500 \mathrm{MHz}, \mathrm{CDCl}_{3}\right): \delta=7.58-7.49(\mathrm{~m}, 2 \mathrm{H}), 6.87-6.83(\mathrm{~m}, 2 \mathrm{H}), 4.80(\mathrm{~d}, 1 \mathrm{H}$, $J=1.6 \mathrm{~Hz}), 4.33(\mathrm{~d}, 1 \mathrm{H}, J=1.6 \mathrm{~Hz}), 3.82(\mathrm{~s}, 3 \mathrm{H}), 0.26(\mathrm{~s}, 9 \mathrm{H})$ ppm. ${ }^{13} \mathrm{C}$ NMR $(125 \mathrm{MHz}$, $\left.\mathrm{CDCl}_{3}\right): \delta=159.8,155.5,130.4,126.7,113.5,89.6,55.4,0.3 \mathrm{ppm}$.

The data were in accordance with literature values. ${ }^{[9]}$ 


\section{Synthesis of the tricarbonyl compounds}

ethyl 2-oxo-1-(2-oxopropyl)cyclopentane-1-carboxylate (2a)<smiles>CCOC(=O)C1(CC(C)=O)CCCC1=O</smiles>

According to the general procedure $B$ the product was synthesized from ethyl 2-oxocyclopentane-1-carboxylate (1a, $78 \mathrm{mg}, 0.50 \mathrm{mmol}, 1.0$ equiv) and 2-trimethylsilyloxy-propene (326 mg, $2.50 \mathrm{mmol}, 5.0$ equiv). After purification by column chromatography $\left(\mathrm{SiO}_{2}\right.$, $n$-pentane:diethyl ether $=2: 1)$ the product $(81 \mathrm{mg}, 0.38 \mathrm{mmol}, 76 \%)$ was obtained as a colorless oil.

$\mathbf{R}_{\boldsymbol{f}}\left(\mathrm{SiO}_{2}, n\right.$-pentane:diethyl ether $\left.=2: 1\right)=0.22$.

${ }^{1} \mathrm{H}$ NMR $\left(300 \mathrm{MHz}, \mathrm{CDCl}_{3}\right): \delta=4.14\left(\mathrm{q}, 2 \mathrm{H},{ }^{3} \mathrm{~J}=7.1 \mathrm{~Hz}\right), 3.21\left(\mathrm{~d}, 1 \mathrm{H},{ }^{2} \mathrm{~J}=18.7 \mathrm{~Hz}\right), 2.95(\mathrm{~d}$, $\left.1 \mathrm{H},{ }^{2} J=18.7 \mathrm{~Hz}\right), 2.61-2.43(\mathrm{~m}, 3 \mathrm{H}), 2.14(\mathrm{~s}, 3 \mathrm{H}), 2.11-1.96(\mathrm{~m}, 3 \mathrm{H}), 1.23(\mathrm{t}, 3 \mathrm{H}$, $\left.{ }^{3} \mathrm{~J}=7.1 \mathrm{~Hz}\right)$ ppm. ${ }^{13} \mathrm{C}$ NMR $\left(75 \mathrm{MHz}, \mathrm{CDCl}_{3}\right): \delta=215.0,205.5,170.7,61.8,57.5,47.7,37.8$, 33.4, 30.2, 19.9, $14.1 \mathrm{ppm}$.

The data were in accordance with literature values. ${ }^{[10]}$

isopropyl 2-oxo-1-(2-oxopropyl)cyclopentane-1-carboxylate (2b)<smiles>CCCCC1(C(=O)OCC)CCCC1=O</smiles>

According to the general procedure $B$ the product was synthesized from isopropyl 2-oxocyclopentane-1-carboxylate ( $85 \mathrm{mg}, 0.50 \mathrm{mmol}, 1.0$ equiv) and 2-trimethylsilyloxypropene (326 mg, $2.50 \mathrm{mmol}, 5.0$ equiv). After purification by column chromatography $\left(\mathrm{SiO}_{2}, n\right.$-pentane:diethyl ether $=3: 1 \rightarrow 1: 1$ ) the product ( $56 \mathrm{mg}, 0.25 \mathrm{mmol}, 49 \%$ ) was obtained as a colorless oil.

$\mathbf{R}_{\boldsymbol{f}}\left(\mathrm{SiO}_{2}, n\right.$-pentane:diethyl ether $\left.=3: 1\right)=0.52$.

${ }^{1} \mathrm{H}$ NMR $\left(500 \mathrm{MHz}, \mathrm{CDCl}_{3}\right): \delta=4.96$ (sept, $\left.1 \mathrm{H},{ }^{3} \mathrm{~J}=6.2 \mathrm{~Hz}\right), 3.17\left(\mathrm{~d}, 1 \mathrm{H},{ }^{2} \mathrm{~J}=18.7 \mathrm{~Hz}\right), 2.93$ $\left(\mathrm{d}, 1 \mathrm{H},{ }^{2} \mathrm{~J}=18.7 \mathrm{~Hz}\right), 2.54-2.38(\mathrm{~m}, 3 \mathrm{H}), 2.12(\mathrm{~s}, 3 \mathrm{H}), 2.11-1.96(\mathrm{~m}, 3 \mathrm{H}), 1.21(\mathrm{~d}, 3 \mathrm{H}$, $\left.{ }^{3} \mathrm{~J}=6.3 \mathrm{~Hz}\right), 1.17\left(\mathrm{~d}, 3 \mathrm{H},{ }^{3} \mathrm{~J}=6.3 \mathrm{~Hz}\right)$ ppm. ${ }^{13} \mathrm{C} \mathrm{NMR}\left(125 \mathrm{MHz}, \mathrm{CDCl}_{3}\right): \delta=215.0,205.5$, 170.2, 69.3, 57.5, 47.5, 37.7, 33.4, 30.1, 21.5, 19.9 ppm. IR (ATR, neat): $\lambda^{-1}=2981(\mathrm{w}), 1749$ (m), $1715(\mathrm{~s}), 1454(w), 1402(w), 1374(w), 1230(w), 1167(w), 1147(w), 1101(s), 912(m)$, 
$856(w), 655(w), 608(w) \mathrm{cm}^{-1}$. GC/MS (El): m/z (\%) = $226\left(\left[\mathrm{M}^{+}\right], 3\right), 183$ (15), 167 (48), 166 (100), 141 (58), 139 (63), 138 (23), 127 (15), 124 (66), 123 (50), 113 (36), 111 (58), 110 (18), 97 (47), 95 (48), 67 (22). HRMS (EI): calculated $\mathrm{C}_{12} \mathrm{H}_{18} \mathrm{O}_{4}{ }^{+}$: 226.1200, found: 226.1205.

ethyl 2-oxo-1-(2-oxopropyl)cyclohexane-1-carboxylate (2e)<smiles>CCOC(=O)C1(CC(C)=O)CCCCC1=O</smiles>

According to the general procedure $B$ the product was synthesized from ethyl 2-oxocyclohexane-1-carboxylate ( $85 \mathrm{mg}, 0.50 \mathrm{mmol}, 1.0$ equiv) and 2-trimethylsilyloxypropene (326 mg, $2.50 \mathrm{mmol}, 5.0$ equiv). After purification by column chromatography $\left(\mathrm{SiO}_{2}, n\right.$-pentane:diethyl ether $=2: 1)$ the product $(91 \mathrm{mg}, 0.40 \mathrm{mmol}, 80 \%)$ was obtained as a colorless oil.

$\mathbf{R}_{\boldsymbol{f}}\left(\mathrm{SiO}_{2}, n\right.$-pentane:diethyl ether $\left.=2: 1\right)=0.28$.

${ }^{1}$ H NMR $\left(500 \mathrm{MHz}, \mathrm{CDCl}_{3}\right): \delta=4.25-4.15(\mathrm{~m}, 2 \mathrm{H}), 2.86-2.72(\mathrm{~m}, 3 \mathrm{H}), 2.48-2.41(\mathrm{~m}, 1 \mathrm{H})$, 2.38-2.31 (m, $1 \mathrm{H}), 2.17(\mathrm{~s}, 3 \mathrm{H}), 2.07-1.93(\mathrm{~m}, 1 \mathrm{H}), 1.75-1.65(\mathrm{~m}, 4 \mathrm{H}), 1.25(\mathrm{t}, 3 \mathrm{H}$, ${ }^{3} \mathrm{~J}=7.2 \mathrm{~Hz}$ ) ppm. ${ }^{13} \mathrm{C}$ NMR $\left(125 \mathrm{MHz}, \mathrm{CDCl}_{3}\right): \delta=207.6,205.6,172.0,61.6,59.3,48.2,40.6$, 36.9, 30.5, 27.0, 22.0, $14.1 \mathrm{ppm}$.

The data were in accordance with literature values. ${ }^{[10]}$

methyl 2-oxo-1-(2-oxopropyl)cycloheptane-1-carboxylate (2f)<smiles>COC(=O)C1(CC(C)=O)CCCCCC1=O</smiles>

According to the general procedure $B$ the product was synthesized from methyl 2-oxocycloheptane-1-carboxylate (51 mg, $0.30 \mathrm{mmol}, 1.0$ equiv) and 2-trimethylsilyloxypropene (195 mg, $1.50 \mathrm{mmol}, 5.0$ equiv). After purification by column chromatography $\left(\mathrm{SiO}_{2}, n\right.$-pentane:diethyl ether $=3: 1 \rightarrow 1: 1)$ the product $(41 \mathrm{mg}, 0.18 \mathrm{mmol}, 60 \%)$ was obtained as a colorless oil.

$\mathbf{R}_{\boldsymbol{f}}\left(\mathrm{SiO}_{2}, n\right.$-pentane:diethyl ether $\left.=3: 1\right)=0.31$.

${ }^{1} \mathrm{H}$ NMR $\left(300 \mathrm{MHz}, \mathrm{CDCl}_{3}\right): \delta=3.72(\mathrm{~s}, 3 \mathrm{H}), 3.18\left(\mathrm{~d}, 1 \mathrm{H},{ }^{2} \mathrm{~J}=17.4 \mathrm{~Hz}\right), 2.85(\mathrm{~m}, 1 \mathrm{H}), 2.74$ $\left(\mathrm{d}, 1 \mathrm{H},{ }^{2} \mathrm{~J}=17.4 \mathrm{~Hz}\right), 2.58-2.49(\mathrm{~m}, 1 \mathrm{H}), 2.18(\mathrm{~s}, 3 \mathrm{H}), 2.17-2.11(\mathrm{~m}, 1 \mathrm{H}), 2.05-1.97(\mathrm{~m}, 1 \mathrm{H})$, 1.89-1.80 (m, $1 \mathrm{H}), 1.78-1.65(\mathrm{~m}, 2 \mathrm{H}), 1.53-1.47(\mathrm{~m}, 2 \mathrm{H}), 1.31-1.26(\mathrm{~m}, 1 \mathrm{H}) \mathrm{ppm} .{ }^{13} \mathrm{C}$ NMR 
$\left(75 \mathrm{MHz}, \mathrm{CDCl}_{3}\right): \delta=209.4,205.9,172.7,61.6,52.6,48.5,42.0,33.6,30.5,30.4,26.4,24.9$ ppm.

The data were in accordance with literature values. ${ }^{[10]}$

\section{2-acetyl-2-(2-oxopropyl)cyclopentane-1-one (2g)}<smiles>CC(=O)CC1(C(C)=O)CCCC1=O</smiles>

According to the general procedure B the product was synthesized from 2-acetylcyclopentan1-one (63 mg, $0.50 \mathrm{mmol}, 1.0$ equiv) and 2-trimethylsilyloxypropene (326 mg, $2.50 \mathrm{mmol}$, 5.0 equiv). After purification by column chromatography $\left(\mathrm{SiO}_{2}, n\right.$-pentane:diethyl ether $=$ $3: 1 \rightarrow 1: 1$ ) the product ( $55 \mathrm{mg}, 0.30 \mathrm{mmol}, 52 \%$ ) was obtained as a colorless solid.

$\mathbf{R}_{\boldsymbol{f}}\left(\mathrm{SiO}_{2}, n\right.$-pentane:diethyl ether $\left.=3: 1\right)=0.11$.

${ }^{1} \mathrm{H}$ NMR $\left(300 \mathrm{MHz}, \mathrm{CDCl}_{3}\right): \delta=3.13\left(\mathrm{~d}, 1 \mathrm{H},{ }^{2} \mathrm{~J}=18.5 \mathrm{~Hz}\right), 2.97\left(\mathrm{~d}, 1 \mathrm{H},{ }^{2} J=18.5 \mathrm{~Hz}\right), 2.70-$ $2.64(\mathrm{~m}, 1 \mathrm{H})$, 2.53-2.42 (m, $1 \mathrm{H})$, 2.39-2.31 (m, $1 \mathrm{H}), 2.14$ (s, $3 \mathrm{H}), 2.13(\mathrm{~s}, 3 \mathrm{H})$, 2.02-1.90 (m, $2 \mathrm{H}), 1.88-1.80(\mathrm{~m}, 1 \mathrm{H}) \mathrm{ppm} .{ }^{13} \mathrm{C}$ NMR $\left(125 \mathrm{MHz}, \mathrm{CDCl}_{3}\right): \delta=215.6,205.2,202.9,65.8,48.6$, 38.1, 31.7, 30.2, 26.4, 19.8 ppm. IR (ATR, neat): $\lambda^{-1}=2959(\mathrm{w}), 1737(\mathrm{~m}), 1701(\mathrm{~s}), 1403(\mathrm{~m})$, $1358(\mathrm{~m}), 1165(\mathrm{~m}), 1144(\mathrm{~m}), 1113(\mathrm{~m}), 1053(\mathrm{w}), 982(\mathrm{w}), 823(\mathrm{w}), 682(\mathrm{w}), 617(\mathrm{w}), 603(\mathrm{w})$, $575(\mathrm{~m}), 563(\mathrm{~m}) \mathrm{cm}^{-1}$. GC/MS (El): m/z (\%) = $182\left(\left[\mathrm{M}^{+}\right], 0.13\right), 164$ (1), 140 (56), 139 (16), 122 (7), 111 (9), 97 (100), 85 (13), 79 (9), 69 (10), 55 (10). HRMS (ESI,+): m/z calculated for $\mathrm{C}_{10} \mathrm{H}_{15} \mathrm{O}_{3}{ }^{+}: 183.1015$ found: 183.1028 .

The compound is literature known, but has not been characterised sufficiently. ${ }^{[11]}$

methyl 1-oxo-2-(2-propyl)-2,3-dihydro-1 $H$-indene-2-carboxylate (2i)<smiles>COC(=O)C1(CC(C)=O)Cc2ccccc2C1=O</smiles>

According to the general procedure B the product was synthesized from methyl 1-oxo-2,3dihydro- $1 \mathrm{H}$-indene-2-carboxylate $\quad(48 \mathrm{mg}, \quad 0.25 \mathrm{mmol}, \quad 1.0$ equiv) and 2trimethylsilyloxypropene (163 mg, $1.25 \mathrm{mmol}, 5.0$ equiv). After purification by column chromatography $\left(\mathrm{SiO}_{2}, n\right.$-pentane:diethyl ether $\left.=1: 1\right)$ the product $(53 \mathrm{mg}, 0.22 \mathrm{mmol}, 88 \%)$ was obtained as a colorless oil. 
$\mathbf{R}_{\boldsymbol{f}}\left(\mathrm{SiO}_{2}, n\right.$-pentane:diethyl ether $\left.=1: 1\right)=0.38$.

${ }^{1} \mathrm{H}$ NMR $\left(300 \mathrm{MHz}, \mathrm{CDCl}_{3}\right): \delta=7.76\left(\mathrm{~d}, 1 \mathrm{H},{ }^{3} \mathrm{~J}=7.7 \mathrm{~Hz}\right), 7.63\left(\mathrm{dt}, 1 \mathrm{H},{ }^{3} \mathrm{~J}=7.5 \mathrm{~Hz}\right.$, $\left.{ }^{4} J=1.2 \mathrm{~Hz}\right), 7.48\left(\mathrm{~d}, 1 \mathrm{H},{ }^{3} \mathrm{~J}=7.7 \mathrm{~Hz}\right), 7.39\left(\mathrm{t}, 1 \mathrm{H},{ }^{3} J=7.5 \mathrm{~Hz}\right), 3.95\left(\mathrm{~d}, 1 \mathrm{H},{ }^{2} J=17.6 \mathrm{~Hz}\right)$, $3.64(\mathrm{~s}, 3 \mathrm{H}), 3.59\left(\mathrm{~d}, 1 \mathrm{H},{ }^{2} \mathrm{~J}=18.5 \mathrm{~Hz}\right), 3.08\left(\mathrm{~d}, 1 \mathrm{H},{ }^{2} \mathrm{~J}=17.6 \mathrm{~Hz}\right), 2.82\left(\mathrm{~d}, 1 \mathrm{H},{ }^{2} \mathrm{~J}=18.5 \mathrm{~Hz}\right)$, $2.19(\mathrm{~s}, 3 \mathrm{H}) \mathrm{ppm} .{ }^{13} \mathrm{C}$ NMR $\left(75 \mathrm{MHz}, \mathrm{CDCl}_{3}\right): \delta=205.6,201.6,170.4,153.8,135.7,134.7$, 127.9, 126.5, 125.0, 58.3, 53.1, 48.1, 38.0, 30.1 ppm.

The data were in accordance with literature values. ${ }^{[10]}$

methyl 4-bromo-1-oxo-2-(2-oxopropyl)-2,3-dihydro-1 H-indene-2-carboxylate (2j)<smiles>COC(=O)C1(CC(C)=O)Cc2c(Br)cccc2C1=O</smiles>

According to the general procedure $B$ the product was synthesized from methyl 4-bromo-1oxo-2,3-dihydro- $1 \mathrm{H}$-indene-2-carboxylate $(67 \mathrm{mg}, 0.25 \mathrm{mmol}, 1.0$ equiv) and 2-trimethylsilyloxypropene (163 mg, $1.25 \mathrm{mmol}, 5.0$ equiv). After purification by column chromatography $\left(\mathrm{SiO}_{2}, n\right.$-pentane:diethyl ether $\left.=2: 1\right)$ the product $(46 \mathrm{mg}, 0.14 \mathrm{mmol}, 56 \%)$ was obtained as a colorless solid.

$\mathbf{R}_{\boldsymbol{f}}\left(\mathrm{SiO}_{2}, n\right.$-pentane:diethyl ether $\left.=2: 1\right)=0.29$.

${ }^{1} \mathrm{H}$ NMR $\left(500 \mathrm{MHz}, \mathrm{CDCl}_{3}\right): \delta=7.80\left(\mathrm{dd}, 1 \mathrm{H},{ }^{3} \mathrm{~J}=7.8 \mathrm{~Hz},{ }^{4} \mathrm{~J}=0.9 \mathrm{~Hz}\right), 7.72(\mathrm{~d}, 1 \mathrm{H}$, $\left.{ }^{3} \mathrm{~J}=7.7 \mathrm{~Hz}\right), 7.31\left(\mathrm{t}, 1 \mathrm{H},{ }^{3} \mathrm{~J}=7.7 \mathrm{~Hz}\right), 3.82\left(\mathrm{~d}, 1 \mathrm{H},{ }^{2} \mathrm{~J}=18.2 \mathrm{~Hz}\right), 3.66(\mathrm{~s}, 3 \mathrm{H}), 3.57(\mathrm{~d}, 1 \mathrm{H}$, $\left.{ }^{2} \mathrm{~J}=18.6 \mathrm{~Hz}\right), 2.99\left(\mathrm{~d}, 1 \mathrm{H},{ }^{2} \mathrm{~J}=18.2 \mathrm{~Hz}\right), 2.96\left(\mathrm{~d}, 1 \mathrm{H},{ }^{2} \mathrm{~J}=18.5 \mathrm{~Hz}\right), 2.20(\mathrm{~s}, 3 \mathrm{H}) \mathrm{ppm}$. ${ }^{13} \mathrm{C} \mathrm{NMR}\left(125 \mathrm{MHz}, \mathrm{CDCl}_{3}\right): \delta=205.3,201.0,170.0,153.2,138.3,136.9,129.6,123.7,122.0$, 58.2, 53.3, 48.1, 39.1, 30.0 ppm.

The data were in accordance with literature values. ${ }^{[10]}$

methyl 5,6-dimethoxy-1-oxo-2-(2-oxopropyl)-2,3-dihydro-1 H-indene-2-carboxylate (2k)<smiles>COC(=O)C1(CC(C)=O)Cc2cc(OC)c(OC)cc2C1=O</smiles>

According to the general procedure $B$ the product was synthesized from methyl 5,6-dimethoxy1-oxo-2,3-dihydro-1 $\mathrm{H}$-indene-2-carboxylate $(63 \mathrm{mg}, 0.25 \mathrm{mmol}, 1.0$ equiv) and 2-trimethyl- 
silyloxypropene (163 mg, $1.25 \mathrm{mmol}, 5.0$ equiv). After purification by column chromatography $\left(\mathrm{SiO}_{2}, n\right.$-pentane:diethyl ether $\left.=1: 5\right)$ the product $(46 \mathrm{mg}, 0.14 \mathrm{mmol}, 56 \%)$ was obtained as a colorless solid.

$\mathbf{R}_{\boldsymbol{f}}\left(\mathrm{SiO}_{2}, n\right.$-pentane:diethyl ether $\left.=1: 5\right)=0.22$.

${ }^{1} \mathrm{H}$ NMR $\left(500 \mathrm{MHz}, \mathrm{CDCl}_{3}\right): \delta=7.16(\mathrm{~s}, 1 \mathrm{H}), 6.89(\mathrm{~s}, 1 \mathrm{H}), 3.98(\mathrm{~s}, 3 \mathrm{H}), 3.90(\mathrm{~s}, 3 \mathrm{H}), 3.89$ $3.86(\mathrm{~m}, 1 \mathrm{H}), 3.65(\mathrm{~s}, 3 \mathrm{H}), 3.61\left(\mathrm{~d}, 1 \mathrm{H},{ }^{2} \mathrm{~J}=18.5 \mathrm{~Hz}\right), 2.98\left(\mathrm{~d}, 1 \mathrm{H},{ }^{2} \mathrm{~J}=17.5 \mathrm{~Hz}\right), 2.73(\mathrm{~d}$, $1 \mathrm{H},{ }^{2} \mathrm{~J}=18.4 \mathrm{~Hz}$ ), $2.20(\mathrm{~s}, 3 \mathrm{H}) \mathrm{ppm} .{ }^{13} \mathrm{C}$ NMR $\left(125 \mathrm{MHz}, \mathrm{CDCl}_{3}\right): \delta=205.9,199.9,170.7$, 156.5, 149.9, 149.6, 127.2, 107.3, 105.1, 58.7, 56.5, 56.3, 53.1, 48.2, 37.8, 30.2 ppm.

The data were in accordance with literature values. ${ }^{[10]}$

ethyl 1-oxo-2-(2-oxopropyl)-1,2,3,4-tetrahydronaphtalene-2-carboxylate (2l)<smiles>CCOC(=O)C1(CC(C)=O)CCc2ccccc2C1=O</smiles>

According to the general procedure $B$ the product was synthesized from ethyl 1-oxo-1,2,3,4tetrahydronaphthalene-2-carboxylate (109 mg, $0.5 \mathrm{mmol}, 1.0$ equiv) and 2-trimethylsilyloxypropene (326 mg, $2.5 \mathrm{mmol}, 5.0$ equiv). After purification by column chromatography $\left(\mathrm{SiO}_{2}\right.$, $n$-pentane:diethyl ether $=1: 1)$ the product $(66 \mathrm{mg}, 0.24 \mathrm{mmol}, 48 \%)$ was obtained as a colorless oil.

$\mathbf{R}_{\boldsymbol{f}}\left(\mathrm{SiO}_{2}, n\right.$-pentane:diethyl ether $\left.=1: 1\right)=0.25$.

${ }^{1} \mathrm{H}$ NMR $\left(300 \mathrm{MHz}, \mathrm{CDCl}_{3}\right): \delta=8.01\left(\mathrm{~d}, 1 \mathrm{H},{ }^{3} \mathrm{~J}=7.8 \mathrm{~Hz}\right), 7.45\left(\mathrm{t}, 1 \mathrm{H},{ }^{3} \mathrm{~J}=7.5 \mathrm{~Hz}\right), 7.30(\mathrm{t}$, $\left.1 \mathrm{H},{ }^{3} \mathrm{~J}=7.7 \mathrm{~Hz}\right), 7.20\left(\mathrm{~d}, 1 \mathrm{H},{ }^{3} \mathrm{~J}=7.6 \mathrm{~Hz}\right), 4.14\left(\mathrm{q}, 2 \mathrm{H},{ }^{3} \mathrm{~J}=7.2 \mathrm{~Hz}\right), 3.13-2.94(\mathrm{~m}, 3 \mathrm{H}), 2.86$ $\left(\mathrm{dt}, 1 \mathrm{H},{ }^{3} \mathrm{~J}=4.3 \mathrm{~Hz},{ }^{2} \mathrm{~J}=17.3 \mathrm{~Hz}\right), 2.52-2.36(\mathrm{~m}, 2 \mathrm{H}), 2.20(\mathrm{~s}, 3 \mathrm{H}), 1.13\left(\mathrm{t}, 3 \mathrm{H},{ }^{3} \mathrm{~J}=7.2 \mathrm{~Hz}\right)$ ppm. ${ }^{13} \mathrm{C}$ NMR $\left(75 \mathrm{MHz}, \mathrm{CDCl}_{3}\right): \delta=205.3,195.1,171.9,143.0,133.5,132.2,128.7,128.0$, $126.9,61.7,56.4,47.5,31.9,30.6,26.2,14.0$ ppm.

The data were in accordance with literature values. ${ }^{[10]}$ 
<smiles>COC(=O)C1(CC(C)=O)CCc2cc(OC)ccc2C1=O</smiles>

According to the general procedure $B$ the product was synthesized from methyl 6-methoxy-1oxo-1,2,3,4-tetrahydronaphthalene-2-carboxylate (59 mg, $0.25 \mathrm{mmol}, 1.0$ equiv) and 2trimethyl-silyloxypropene (163 mg, $1.25 \mathrm{mmol}, 5.0$ equiv). After purification by column chromatography $\left(\mathrm{SiO}_{2}, n\right.$-pentane:diethyl ether $\left.=1: 2\right)$ the product $(39 \mathrm{mg}, 0.13 \mathrm{mmol}, 52 \%)$ was obtained as a colorless solid.

$\mathbf{R}_{\boldsymbol{f}}\left(\mathrm{SiO}_{2}, n\right.$-pentane:diethyl ether $\left.=1: 2\right)=0.29$.

${ }^{1} \mathrm{H}$ NMR $\left(500 \mathrm{MHz}, \mathrm{CDCl}_{3}\right): \delta=8.00\left(\mathrm{~d}, 1 \mathrm{H},{ }^{3} \mathrm{~J}=8.8 \mathrm{~Hz}\right), 6.83\left(\mathrm{dd}, 1 \mathrm{H},{ }^{3} \mathrm{~J}=8.8 \mathrm{~Hz}\right.$, ${ }^{4} \mathrm{~J}=2.5 \mathrm{~Hz}$ ), $6.66\left(\mathrm{~d}, 1 \mathrm{H},{ }^{4} \mathrm{~J}=2.4 \mathrm{~Hz}\right.$ ), $3.85(\mathrm{~s}, 3 \mathrm{H}), 3.68(\mathrm{~s}, 3 \mathrm{H}), 3.05-2.99(\mathrm{~m}, 3 \mathrm{H}), 2.83$ (dt, $\left.1 \mathrm{H},{ }^{2} \mathrm{~J}=17.2 \mathrm{~Hz},{ }^{3} \mathrm{~J}=4.3 \mathrm{~Hz}\right), 2.47-2.42(\mathrm{~m}, 2 \mathrm{H}), 2.22(\mathrm{~s}, 3 \mathrm{H}) \mathrm{ppm} .{ }^{13} \mathrm{C}$ NMR $(125 \mathrm{MHz}$, $\left.\mathrm{CDCl}_{3}\right): \delta=205.5,193.6,172.6,163.8,145.7,130.6,125.6,113.6,112.6,56.3,55.6,52.8$, 47.6, 31.9, 30.7, $26.7 \mathrm{ppm}$.

The data were in accordance with literature values. ${ }^{[10]}$

methyl 1-oxo-2-(2-oxoheptyl)-2,3-dihydro-1H-indene-2-carboxylate (2n)<smiles>CCCCCC(=O)CC1(C(=O)OC)Cc2ccccc2C1=O</smiles>

According to the general procedure $B$ the product was synthesized from methyl 1-oxo-2,3dihydro- $1 \mathrm{H}$-indene-2-carboxylate $(48 \mathrm{mg}, 0.25 \mathrm{mmol}, 1.0$ equiv) and 2-trimethylsiloxy-1heptene ( $233 \mathrm{mg}, 1.25 \mathrm{mmol}, 5.0$ equiv). After purification by column chromatography $\left(\mathrm{SiO}_{2}\right.$, $n$-pentane:diethyl ether $=5: 1)$ the product $(32 \mathrm{mg}, 0.11 \mathrm{mmol}, 44 \%)$ was obtained as a colorless oil.

$\mathbf{R}_{\boldsymbol{f}}\left(\mathrm{SiO}_{2}, n\right.$-pentane:diethyl ether $\left.=5: 1\right)=0.18$.

${ }^{1} \mathrm{H}$ NMR $\left(500 \mathrm{MHz}, \mathrm{CDCl}_{3}\right): \delta=7.77\left(\mathrm{~d}, 1 \mathrm{H},{ }^{3} \mathrm{~J}=7.6 \mathrm{~Hz}\right), 7.63\left(\mathrm{dt}, 1 \mathrm{H},{ }^{3} \mathrm{~J}=7.5 \mathrm{~Hz}\right.$, $\left.{ }^{4} \mathrm{~J}=1.0 \mathrm{~Hz}\right), 7.48\left(\mathrm{td}, 1 \mathrm{H},{ }^{3} \mathrm{~J}=7.6 \mathrm{~Hz},{ }^{4} \mathrm{~J}=0.8 \mathrm{~Hz}\right), 7.41-7.38(\mathrm{~m}, 1 \mathrm{H}), 3.95(\mathrm{~d}, 1 \mathrm{H}$, $\left.{ }^{2} J=17.6 \mathrm{~Hz}\right), 3.64(\mathrm{~s}, 3 \mathrm{H}), 3.56\left(\mathrm{~d}, 1 \mathrm{H},{ }^{2} \mathrm{~J}=18.4 \mathrm{~Hz}\right), 3.07\left(\mathrm{~d}, 1 \mathrm{H},{ }^{2} \mathrm{~J}=17.6 \mathrm{~Hz}\right), 2.81(\mathrm{~d}$, 
$\left.1 \mathrm{H},{ }^{2} \mathrm{~J}=18.4 \mathrm{~Hz}\right), 2.51-2.37(\mathrm{~m}, 2 \mathrm{H}), 1.61-1.56(\mathrm{~m}, 2 \mathrm{H}), 1.31-1.24(\mathrm{~m}, 4 \mathrm{H}), 0.88(\mathrm{t}, 3 \mathrm{H}$ ${ }^{3} \mathrm{~J}=7.0 \mathrm{~Hz}$ ) ppm. ${ }^{13} \mathrm{C}$ NMR $\left(125 \mathrm{MHz}, \mathrm{CDCl}_{3}\right): \delta=208.3,201.7,170.5,153.8,135.7,134.8$, 127.9, 126.6, 125.0, 58.3, 53.1, 47.4, 42.8, 38.2, 31.4, 23.6, 22.6, 14.0 ppm.

IR (ATR, neat): $\lambda^{-1}=2954(w), 2929(w), 2860(w), 1741(m), 1709(s), 1608(w), 1464(w)$, $1435(\mathrm{w}), 1277(\mathrm{~m}), 1207(\mathrm{~m}), 1175(\mathrm{~m}), 926(\mathrm{w}), 751(\mathrm{~m}), 565(\mathrm{w}) \mathrm{cm}^{-1}$. GC/MS (EI): m/z $(\%)=302\left(\left[\mathrm{M}^{+}\right], 20\right), 271$ (28), 270 (86), 243 (19), 203 (53), 189 (43), 172 (31), 157 (84), 144 (100), 115 (52), 99 (81), 71 (60). HRMS (EI): m/z calculated for $\mathrm{C}_{18} \mathrm{H}_{22} \mathrm{O}_{4}{ }^{+}:$302.1512, found: 302.1507.

methyl 2-(3,3-dimethyl-2-oxobutyl)-1-oxo-2,3-dihydro-1 H-indene-2-carboxylate (2o)<smiles>COC(=O)C1(CC(=O)C(C)(C)C)Cc2ccccc2C1=O</smiles>

According to the general procedure $B$ the product was synthesized from methyl 1-oxo-2,3dihydro- $1 \mathrm{H}$-indene-2-carboxylate $(48 \mathrm{mg}, \quad 0.25 \mathrm{mmol}, 1.0$ equiv) and 3,3-dimethyl-2trimethylsilyloxy-1-butene $(215 \mathrm{mg}, 1.25 \mathrm{mmol}, 5.0$ equiv). After purification by column chromatography $\left(\mathrm{SiO}_{2}, n\right.$-pentane:diethyl ether $\left.=5: 1 \rightarrow 3: 1\right)$ the product $(33 \mathrm{mg}, 0.11 \mathrm{mmol}$, $46 \%$ ) was obtained as a colorless oil.

$\mathbf{R}_{\boldsymbol{f}}\left(\mathrm{SiO}_{2}, n\right.$-pentane:diethyl ether $\left.=5: 1\right)=0.19$.

'H NMR $\left(500 \mathrm{MHz}, \mathrm{CDCl}_{3}\right): \delta=7.78\left(\mathrm{~d}, 1 \mathrm{H},{ }^{3} \mathrm{~J}=7.7 \mathrm{~Hz}\right), 7.63\left(\mathrm{t}, 1 \mathrm{H},{ }^{3} \mathrm{~J}=7.5 \mathrm{~Hz}\right), 7.49(\mathrm{~d}$, $\left.1 \mathrm{H},{ }^{3} \mathrm{~J}=7.7 \mathrm{~Hz}\right), 7.39\left(\mathrm{t}, 1 \mathrm{H},{ }^{3} \mathrm{~J}=7.5 \mathrm{~Hz}\right), 3.98\left(\mathrm{~d}, 1 \mathrm{H},{ }^{2} \mathrm{~J}=17.5 \mathrm{~Hz}\right), 3.71(\mathrm{~d}, 1 \mathrm{H}$, $\left.{ }^{2} J=18.6 \mathrm{~Hz}\right), 3.64(\mathrm{~s}, 3 \mathrm{H}), 2.96\left(\mathrm{~d}, 1 \mathrm{H},{ }^{2} \mathrm{~J}=17.5 \mathrm{~Hz}\right), 2.90\left(\mathrm{~d}, 1 \mathrm{H},{ }^{2} \mathrm{~J}=18.5 \mathrm{~Hz}\right), 1.17(\mathrm{~s}$, $9 \mathrm{H})$ ppm. ${ }^{13} \mathrm{C}$ NMR $\left(125 \mathrm{MHz}, \mathrm{CDCl}_{3}\right): \delta=213.6,201.9,170.5,153.8,135.6,135.0,127.8$, 126.5, 125.0, 58.4, 53.0, 44.2, 42.6, 38.4, 26.6 ppm.

The data were in accordance with literature values. ${ }^{[10]}$ 


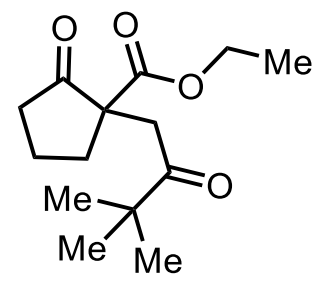

According to the general procedure $B$ the product was synthesized from ethyl 2oxocyclopentane-1-carboxylate (78 $\mathrm{mg}, \quad 0.50 \mathrm{mmol}, \quad 1.0$ equiv) and 3,3-dimethyl-2trimethylsilyloxy-1-butene ( $431 \mathrm{mg}, 2.50 \mathrm{mmol}, 5.0$ equiv). After purification by column chromatography $\left(\mathrm{SiO}_{2}, n\right.$-pentane:diethyl ether $\left.=3: 1\right)$ the product $(51 \mathrm{mg}, 0.20 \mathrm{mmol}, 40 \%)$ was obtained as a colorless oil.

$\mathbf{R}_{\boldsymbol{f}}\left(\mathrm{SiO}_{2}, n\right.$-pentane:diethyl ether $\left.=3: 1\right)=0.47$.

${ }^{1} \mathrm{H}$ NMR $\left(300 \mathrm{MHz}, \mathrm{CDCl}_{3}\right): \delta=4.13\left(\mathrm{q}, 2 \mathrm{H},{ }^{3} \mathrm{~J}=7.2 \mathrm{~Hz}\right), 3.33\left(\mathrm{~d}, 1 \mathrm{H},{ }^{2} \mathrm{~J}=18.7 \mathrm{~Hz}\right), 2.98(\mathrm{~d}$, $\left.1 \mathrm{H},{ }^{2} \mathrm{~J}=18.7 \mathrm{~Hz}\right), 2.60-2.41(\mathrm{~m}, 3 \mathrm{H}), 2.16-2.06(\mathrm{~m}, 1 \mathrm{H}), 2.05-1.96(\mathrm{~m}, 1 \mathrm{H}), 1.96-1.88(\mathrm{~m}$, $1 \mathrm{H}), 1.22\left(\mathrm{t}, 3 \mathrm{H},{ }^{3} \mathrm{~J}=7.1 \mathrm{~Hz}\right), 1.14(\mathrm{~s}, 9 \mathrm{H}) \mathrm{ppm} .{ }^{13} \mathrm{C}$ NMR $\left(125 \mathrm{MHz}, \mathrm{CDCl}_{3}\right): \delta=215.1$, 213.1, 170.9, 61.7, 57.5, 44.1, 42.1, 37.8, 33.5, 26.6, 14.1 ppm.

The data were in accordance with literature values. ${ }^{[12]}$

ethyl 2-oxo-1-(2-oxocyclohexyl)cyclopentane-1-carboxylate (2r)<smiles>CCOC(=O)C1(C2CCCCC2=O)CCCC1=O</smiles>

According to the general procedure B the product was synthesized from ethyl 2oxocyclopentane-1-carboxylate (39 mg, $0.25 \mathrm{mmol}, \quad 1.0 \quad$ equiv) and 1 trimethylsiloxycyclohexene ( $149 \mathrm{mg}, 0.875 \mathrm{mmol}, 3.5$ equiv). After repeated purification by column chromatography $\left(\mathrm{SiO}_{2}, 1 . n\right.$-pentane:diethyl ether $=3: 1,2$. $n$-pentane:diethyl ether $=$ $2: 1,3$. $n$-pentane:diethyl ether $=1: 1)$ the product $(10 \mathrm{mg}, 0.04 \mathrm{mmol}, 16 \%)$ was obtained as a colorless oil.

$\mathbf{R}_{\boldsymbol{f}}\left(\mathrm{SiO}_{2}, n\right.$-pentane:diethyl ether $\left.=3: 1\right)=0.24$.

$\mathbf{R}_{\boldsymbol{f}}\left(\mathrm{SiO}_{2}, n\right.$-pentane:diethyl ether $\left.=2: 1\right)=0.30$.

$\mathbf{R}_{\boldsymbol{f}}\left(\mathrm{SiO}_{2}, n\right.$-pentane:diethyl ether $\left.=1: 1\right)=0.50$.

${ }^{1} \mathrm{H}$ NMR $\left(300 \mathrm{MHz}, \mathrm{CDCl}_{3}\right): \delta=4.34-4.06(\mathrm{~m}, 2 \mathrm{H}), 3.66-3.41(\mathrm{~m}, 1 \mathrm{H}), 2.94-2.68(\mathrm{~m}, 1 \mathrm{H})$, 2.53-2.22 (m, $4 \mathrm{H}), 2.17-1.49(\mathrm{~m}, 9 \mathrm{H}), 1.33-1.19(\mathrm{~m}, 3 \mathrm{H}) \mathrm{ppm} .{ }^{13} \mathrm{C}$ NMR $\left(125 \mathrm{MHz}, \mathrm{CDCl}_{3}\right)$ : 
215.3, 214.4, 213.5, 211.9, 210.1, 209.9, 171.7, 170.0, 169.7, 62.7, 62.3, 61.9, 61.7, 61.5, $56.7,55.9,49.1,42.5,42.2,39.1,37.6,36.0,34.9,30.2,30.1,29.6,28.2,27.8,27.4,25.6$, 25.4. 25.2, 20.2, 18.5, 14.2, $14.1 \mathrm{ppm}$.

Because of the diastereomeric mixture an explicit correlation of the signals was not possible. IR (ATR, neat): $\lambda^{-1}=2939(\mathrm{~m}), 2867(\mathrm{w}), 1754(\mathrm{~m}), 1710(\mathrm{~s}), 1450(\mathrm{~m}), 1406(\mathrm{w}), 1367(\mathrm{w})$, $1228(\mathrm{~m}), 1165(\mathrm{~m}), 1123(\mathrm{~m}), 1028(\mathrm{~m}), 858(\mathrm{w}), 618(\mathrm{w}), 561(\mathrm{w}) \mathrm{cm}^{-1}$. GC/MS (El): m/z $(\%)=252\left(\left[\mathrm{M}^{+}\right], 6\right), 207$ (40), 206 (68), 179 (100), 178 (78), 161 (20), 156 (72), 155 (74), 151 (77), 150 (83), 127 (20), 123 (20), 122 (16), 110 (44), 109 (30), 108 (17). HRMS (El): calculated $\mathrm{C}_{14} \mathrm{H}_{20} \mathrm{O}_{4}{ }^{+}:$252.1356, found: 252.1357.

methyl 1-oxo-2-(2-oxo-2-phenylethyl)2,3-dihdro-1H-indene-2-carboxylate (2s)<smiles>COC(=O)C1(CC(=O)c2ccccc2)Cc2ccccc2C1=O</smiles>

According to the general procedure $B$ the product was synthesized from methyl 1-oxo-2,3dihydro- $1 \mathrm{H}$-indene-2-carboxylate (48 mg, $0.25 \mathrm{mmol}, 1.0$ equiv) and $\alpha$-trimethylsilyloxystyrene (165 mg, $0.858 \mathrm{mmol}, 3.4$ equiv). After purification by column chromatography $\left(\mathrm{SiO}_{2}\right.$, $n$-pentane:diethyl ether $=2: 1)$ the product $(16 \mathrm{mg}, 0.052 \mathrm{mmol}, 20 \%)$ was obtained as a colorless oil.

$\mathbf{R}_{\boldsymbol{f}}\left(\mathrm{SiO}_{2}, n\right.$-pentane:diethyl ether $\left.=2: 1\right)=0.34$.

${ }^{1} \mathrm{H}$ NMR $\left(500 \mathrm{MHz}, \mathrm{CDCl}_{3}\right): \delta=7.96-7.94(\mathrm{~m}, 2 \mathrm{H}), 7.81\left(\mathrm{~d},{ }^{3} \mathrm{~J}=7.6 \mathrm{~Hz}, 1 \mathrm{H}\right.$, 7.67-7.62 (m, $1 \mathrm{H}), 7.60-7.55(\mathrm{~m}, 1 \mathrm{H}), 7.51\left(\mathrm{~d}, 1 \mathrm{H},{ }^{3} \mathrm{~J}=7.6 \mathrm{~Hz}\right), 7.49-7.38(\mathrm{~m}, 3 \mathrm{H}), 4.20(\mathrm{dd}, 1 \mathrm{H}$, $\left.{ }^{2} \mathrm{~J}=18.4 \mathrm{~Hz},{ }^{4} \mathrm{~J}=2.4 \mathrm{~Hz}\right), 4.10\left(\mathrm{~d}, 1 \mathrm{H},{ }^{2} \mathrm{~J}=17.6 \mathrm{~Hz}\right), 3.67(\mathrm{~s}, 3 \mathrm{H}), 3.41\left(\mathrm{dd}, 1 \mathrm{H},{ }^{2} \mathrm{~J}=18.4 \mathrm{~Hz}\right.$, $\left.{ }^{4} \mathrm{~J}=2.4 \mathrm{~Hz}\right), 3.13\left(\mathrm{~d}, 1 \mathrm{H},{ }^{2} \mathrm{~J}=17.6 \mathrm{~Hz}\right) \mathrm{ppm} .{ }^{13} \mathrm{C} \mathrm{NMR}\left(125 \mathrm{MHz}, \mathrm{CDCl}_{3}\right): \delta=201.8,197.0$, $170.5,153.8,136.3,135.7,134.9,133.7,128.8,128.2,127.9,126.6,125.0,58.4,53.2,44.1$, $38.3 \mathrm{ppm}$.

The data were in accordance with literature values. ${ }^{[10]}$ 


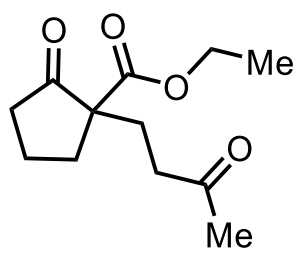

According to the general procedure B the product was synthesized from ethyl 2oxocyclopentane-1-carboxylate (39 mg, $0.25 \mathrm{mmol}, 1.0$ equiv) and 2-trimethylsilyloxy-1,3butadiene (178 mg, $1.25 \mathrm{mmol}, 5.0$ equiv). After purification by column chromatography $\left(\mathrm{SiO}_{2}\right.$, $n$-pentane:diethyl ether $=2: 1)$ the product $(20 \mathrm{mg}, 0.088 \mathrm{mmol}, 35 \%)$ was obtained as a colorless liquid.

$\mathbf{R}_{\boldsymbol{f}}\left(\mathrm{SiO}_{2}, n\right.$-pentane:diethyl ether $\left.=2: 1\right)=0.18$.

'H NMR $\left.\left(500 \mathrm{MHz}, \mathrm{CDCl}_{3}\right): \delta=4.16\left(\mathrm{qd}, 2 \mathrm{H},{ }^{3} \mathrm{~J}=7.2 \mathrm{~Hz},{ }^{2} \mathrm{~J}=1.3 \mathrm{~Hz}\right), 2.75-2.64, \mathrm{~m}, 1 \mathrm{H}\right)$, 2.52-2.43 (m, $2 \mathrm{H})$, 2.42-2.36 (m, $1 \mathrm{H}), 2.34-2.24(\mathrm{~m}, 1 \mathrm{H}), 2.13(\mathrm{~s}, 3 \mathrm{H}), 2.12-2.06(\mathrm{~m}, 1 \mathrm{H})$, 2.04-2.183 (m, $4 \mathrm{H}), 1.24\left(\mathrm{t}, 3 \mathrm{H},{ }^{3} \mathrm{~J}=7.1 \mathrm{~Hz}\right)$ ppm. ${ }^{13} \mathrm{C} \mathbf{N M R}\left(125 \mathrm{MHz}, \mathrm{CDCl}_{3}\right): \delta=215.1$, 208.0, 171.6, 61.6, 59.1, 39.0, 38.1, 34.6, 30.1, 27.1, 19.7, 14.2 ppm.

The data were in accordance with literature values. ${ }^{[13-14]}$

methyl 1-oxo-2-(3.oxobutyl)-2,3-dihydro-1H-indene-2-carboxylate (2u)<smiles>COC(=O)C1(CCC(C)=O)Cc2ccccc2C1=O</smiles>

Method A: According to the general procedure B the product was synthesized from methyl 1oxo-2,3-dihydro- $1 \mathrm{H}$-indene-2-carboxylate $\quad(48 \mathrm{mg}, \quad 0.25 \mathrm{mmol}, \quad 1.0$ equiv) and 2-trimethylsilyloxy-1,3-butadiene (178 mg, $1.25 \mathrm{mmol}, 5.0$ equiv). After purification by column chromatography $\left(\mathrm{SiO}_{2}, n\right.$-pentane:diethyl ether $\left.=1: 1\right)$ the product $(40 \mathrm{mg}, 0.15 \mathrm{mmol}, 62 \%)$ was obtained as a colorless liquid.

Method B: According to the general procedure B the product was synthesized from methyl 1oxo-2,3-dihydro- $1 H$-indene-2-carboxylate $(48 \mathrm{mg}, 0.25 \mathrm{mmol}, 1.0$ equiv) and methyl vinyl ketone ( $89 \mathrm{mg}, 1.25 \mathrm{mmol}, 5.0$ equiv). After purification by column chromatography $\left(\mathrm{SiO}_{2}\right.$, $n$-pentane:diethyl ether $=1: 1)$ the product $(37 \mathrm{mg}, 0.14 \mathrm{mmol}, 57 \%)$ was obtained as colorless liquid. 
$\mathbf{R}_{\boldsymbol{f}}\left(\mathrm{SiO}_{2}, n\right.$-pentane:diethyl ether $\left.=1: 1\right)=0.22$.

${ }^{1} \mathrm{H}$ NMR $\left(500 \mathrm{MHz}, \mathrm{CDCl}_{3}\right): \delta=7.77\left(\mathrm{~d}, 1 \mathrm{H},{ }^{3} \mathrm{~J}=7.7 \mathrm{~Hz}\right), 7.63\left(\mathrm{dt}, 1 \mathrm{H},{ }^{3} \mathrm{~J}=7.6 \mathrm{~Hz}\right.$, $\left.{ }^{4} J=4.4 \mathrm{~Hz}\right), 7.47\left(\mathrm{~d}, 1 \mathrm{H},{ }^{3} \mathrm{~J}=7.7 \mathrm{~Hz}\right), 7.41\left(\mathrm{t}, 1 \mathrm{H},{ }^{3} \mathrm{~J}=7.6 \mathrm{~Hz}\right), 3.69(\mathrm{~s}, 3 \mathrm{H}), 3.66(\mathrm{~d}, 1 \mathrm{H}$, $\left.{ }^{2} J=17.5 \mathrm{~Hz}\right), 3.03\left(\mathrm{~d}, 1 \mathrm{H},{ }^{2} J=17.4 \mathrm{~Hz}\right), 2.66-2.58(\mathrm{~m}, 1 \mathrm{H}), 2.55-2.46(\mathrm{~m}, 1 \mathrm{H}), 2.26-2.21(\mathrm{~m}$, $2 \mathrm{H}), 2.12$ (s, $3 \mathrm{H}$ ) ppm. ${ }^{13} \mathrm{C}$ NMR $\left(125 \mathrm{MHz}, \mathrm{CDCl}_{3}\right): \delta=207.6,202.4,171.7,152.6,135.7$, 135.2, 128.1, 126.5, 125.0, 59.2, 52.9, 38.9, 38.0, 30.1, 28.7 ppm.

The data were in accordance with literature values. ${ }^{[15]}$

\section{Synthesis of the Tris(pyridine-2-ylmethyl)amine (TPA) ligand:}<smiles>c1ccc(CN(Cc2ccccn2)Cc2ccccn2)nc1</smiles>

According to a literature procedure of RUIZ und KONAR ${ }^{[17]}$ 2-(Chloromethyl)pyridine hydrochloride (2.62 g, $16.0 \mathrm{mmol}, 2.0 \mathrm{eq})$ was dissolved in water $(15 \mathrm{~mL})$ and cooled down to $0{ }^{\circ} \mathrm{C}$. At this temperature first aqueous sodium hydroxide solution $(3 \mathrm{~mL}, 5.3 \mathrm{~mol} / \mathrm{L}, 2.0$ equiv) was added and afterwards a solution of 2-picolylamine ( $865 \mathrm{mg}, 8.00 \mathrm{mmol}, 1.0$ equiv) in $\mathrm{CH}_{2} \mathrm{Cl}_{2}(20 \mathrm{~mL})$ was added. Then, the reaction mixture was warmed to room temperature and stirred for 5 days. During this time, additional aqueous sodium hydroxide solution $(3 \mathrm{~mL}$, $5.3 \mathrm{~mol} / \mathrm{L}, 2.0$ equiv) was added in a way, that the $\mathrm{pH}$-value of the aqueous layer did not exceed 9.5. After completion of the addition the reaction mixture was washed with aqueous sodium hydroxide solution (15\%, $3 \times 15 \mathrm{~mL}$ ). The organic phase was dried over $\mathrm{MgSO}_{4}$, filtered and the solvent was removed under reduced pressure. Afterwards, diethylether $(50 \mathrm{~mL})$ was added to the resulting brown residue and the solution was filtered through a pad of Celite, which was washed with boiling diethylether. After removal of the solvent in vacuo the remained solid was recrystallised from diethylether, so that the product $(1.90 \mathrm{~g}, 6.54 \mathrm{mmol}, 82 \%)$ was obtained as yellowish, crystalline solid.

m.p.: $89-92^{\circ} \mathrm{C}$.

${ }^{1} \mathrm{H}-\mathrm{NMR}\left(500 \mathrm{MHz}, \mathrm{CDCl}_{3}\right): \delta=8.56\left(\mathrm{~d}, 3 \mathrm{H},{ }^{3} \mathrm{~J}=4.7 \mathrm{~Hz}\right), 7.68\left(\mathrm{dt}, 3 \mathrm{H},{ }^{3} \mathrm{~J}=7.6 \mathrm{~Hz}\right.$, $\left.{ }^{4} \mathrm{~J}=1.7 \mathrm{~Hz}\right), 7.61\left(\mathrm{~d}, 3 \mathrm{H},{ }^{3} \mathrm{~J}=7.8 \mathrm{~Hz}\right), 7.17\left(\mathrm{dd}, 3 \mathrm{H},{ }^{3} \mathrm{~J}=7.4 \mathrm{~Hz},{ }^{3} \mathrm{~J}=4.9 \mathrm{~Hz},{ }^{4} \mathrm{~J}=1.1 \mathrm{~Hz}\right)$, $3.93(\mathrm{~s}, 6 \mathrm{H})$ ppm. ${ }^{13} \mathrm{C}-\mathrm{NMR}\left(125 \mathrm{MHz}, \mathrm{CDCl}_{3}\right): \delta=159.5,149.2,136.5,123.1,122.1,60.3$ ppm.

The data were in accordance with literature values. ${ }^{[17]}$ 


\section{Synthesis of the manganese-TPA-complex}

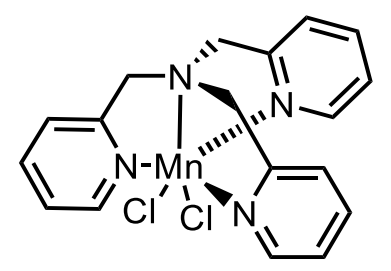

According to a literature procedure HITOMI and FUNABIKI ${ }^{[18]} \mathrm{MnCl}_{2} \cdot 4 \mathrm{H}_{2} \mathrm{O}(198 \mathrm{mg}, 1.00 \mathrm{mmol}$, 1.0 equiv) was dissolved in methanol $(5 \mathrm{~mL})$ and warmed to $50^{\circ} \mathrm{C}$. At this temperature a solution of tris-(pyridine-2-ylmethyl)amine $(290 \mathrm{mg}, 1.00 \mathrm{mmol}, 1.0$ equiv) in methanol $(5 \mathrm{~mL})$ was added. After stirring for $1 \mathrm{~h}$ at $50^{\circ} \mathrm{C}$, the reaction mixture was allowed to cool down to room temperature. After removal of the solvent under reduced pressure the manganese-TPAcomplex was obtained as light brown solid (426 mg, $1.00 \mathrm{mmol}, 100 \%)$.

IR (ATR, neat): $\lambda^{-1}=3422(w), 3060(w), 2941(w), 1601(s), 1572(\mathrm{~m}), 1479(\mathrm{~m}), 1439(\mathrm{~s})$, $1291(\mathrm{~m}), 1154(\mathrm{~m}), 1119$ (w), 1097 (m), 1050 (s), 1015 (s), 961 (w), 911 (w), 762 (s), 638 (m) $\mathrm{cm}^{-1}$.

HRMS (ESI, +): calculated for $\mathrm{C}_{18} \mathrm{H}_{18} \mathrm{CIMnN}_{4}{ }^{+}: 380.0595$, found: 380.0589 .

The data were in accordance with literature values. ${ }^{[19]}$

\section{Synthesis of the chiral ligand}

\section{3,7-bis[(R)-1-phenylethyl]-3,7-diaza-1,5(2,6)-dipyridinacyclooctaphane (8)}

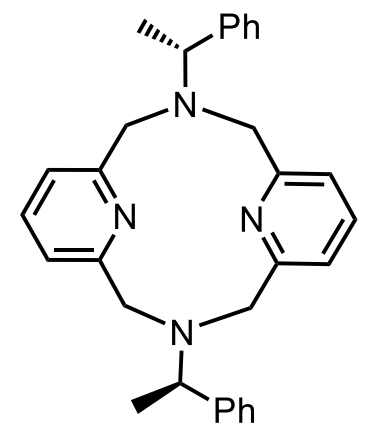

According to a literature procedure of KHUSNUTDINOVA ${ }^{[16]}\left(1 R, 1^{\prime} R\right)-N, N$-[pyridine-2,6diylbis(methylene)]bis(1-phenylethan-1-amine) (399 mg, $1.15 \mathrm{mmol}, 1.05$ equiv) was dissolved in toluene $(4 \mathrm{~mL} / \mathrm{mmol})$ and an aqueous $\mathrm{NaHCO}_{3}$ solution $(10 \%, 6 \mathrm{~mL} / \mathrm{mmol})$ was added. The mixture was heated to $80^{\circ} \mathrm{C}$ and a solution of 2,6-bis(bromomethyl)pyridine (292 mg, $1.10 \mathrm{mmol}, 1.00$ equiv) in toluene was added over a period of 40 minutes. Afterwards, the reaction mixture was stirred over night at $80^{\circ} \mathrm{C}$. After cooling down to room temperature, 
the phases were separated and the water layer was extracted with toluene $(3 \times 10 \mathrm{~mL})$. The combined organic layers were dried over $\mathrm{NaSO}_{4}$ and filtrated. After evaporation of the solvent, the crude product was recrystallized from $\mathrm{CH}_{2} \mathrm{Cl}_{2} / n$-pentane. After filtration and drying in vacuo the product 8 (205 mg, 40\%) was obtained as light yellowish solid.

$[\alpha]^{20}{ }_{D}=101.75$ in $\mathrm{CH}_{2} \mathrm{Cl}_{2} .{ }^{1} \mathrm{H}$ NMR $\left(500 \mathrm{MHz}, \mathrm{CDCl}_{3}\right): \delta=7.56-7.51(\mathrm{~m}, 2 \mathrm{H}), 7.43-7.37(\mathrm{~m}$, $7 \mathrm{H}), 7.28-7.23(\mathrm{~m}, 4 \mathrm{H}), 7.20-7.13(\mathrm{~m}, 3 \mathrm{H}), 3.89\left(\mathrm{~d}, 2 \mathrm{H},{ }^{3} \mathrm{~J}=6.3 \mathrm{~Hz}\right), 3.79(\mathrm{~d}, 4 \mathrm{H}$, $\left.{ }^{2} \mathrm{~J}=14.9 \mathrm{~Hz}\right), 3.64\left(\mathrm{~d}, 4 \mathrm{H},{ }^{2} \mathrm{~J}=15.0 \mathrm{~Hz}\right), 1.39\left(\mathrm{~d}, 6 \mathrm{H},{ }^{3} \mathrm{~J}=6.7 \mathrm{~Hz}\right)$ ppm. ${ }^{13} \mathrm{C} \mathbf{N M R}(125 \mathrm{MHz}$, $\left.\mathrm{CDCl}_{3}\right): \delta=159.9,143.1,136.8,128.2,127.9,126.9,120.6,58.1,56.4,14.7$ ppm. IR (ATR, neat): $\lambda^{-1}=2968(w), 2867(w), 2368(w), 2318(w), 2249(w), 2210(w), 2289(w), 1985(w)$, $1590(\mathrm{~m}), 1574(\mathrm{~m}), 1492(\mathrm{w}), 1453(\mathrm{~m}), 1374(\mathrm{w}), 1265$ (w), $1204(\mathrm{w}), 1154(\mathrm{w}), 1121(\mathrm{w})$, $1084(w), 762(\mathrm{~m}), 699(\mathrm{~s}), 622(\mathrm{w}) \mathrm{cm}^{-1}$. HRMS (ESI,+): $\mathrm{m} / \mathrm{z}$ calculated for $\mathrm{C}_{30} \mathrm{H}_{33} \mathrm{~N}_{4}{ }^{+}: 449.2700$ found: 449.2705 .

\section{Synthesis of the Manganese complex}

\section{8. $\mathrm{MnCl}_{2}$}

The previously synthesized ligand $(8,66 \mathrm{mg}, 0.15 \mathrm{mmol}, 1.0$ equiv) was dissolved in DCM (4 mL) and a solution of $\mathrm{MnCl}_{2} \cdot 2 \mathrm{H}_{2} \mathrm{O}$ (29 mg, $0.15 \mathrm{mmol}, 1.0$ equiv) in methanol ( $\left.2 \mathrm{~mL}\right)$ was added. Afterwards, the reaction mixture was stirred overnight at room temperature. After removal of the solvent the complex (84 mg, 100\%) was obtained as light yellow solid.

IR (ATR, neat): $\lambda^{-1}=3432(w), 2974(w), 2155(w), 2021(w), 1602(m), 1574(m), 1453(\mathrm{~m})$, $1376(\mathrm{~m}), 1263(\mathrm{~m}), 1085(\mathrm{~m}), 1012(\mathrm{~m}), 782(\mathrm{~m}), 701(\mathrm{~s}) \mathrm{cm}^{-1}$. HRMS (ESI,+): m/z calculated for $\mathrm{C}_{30} \mathrm{H}_{32} \mathrm{~N}_{4} \mathrm{MnCl}^{+}: 538.1693$ found: 538.1691. 


\section{Analytical data of the observed homo-coupling products of the silyl}

enol ethers

\section{1,4-diphenylbutane-1,4-dione}

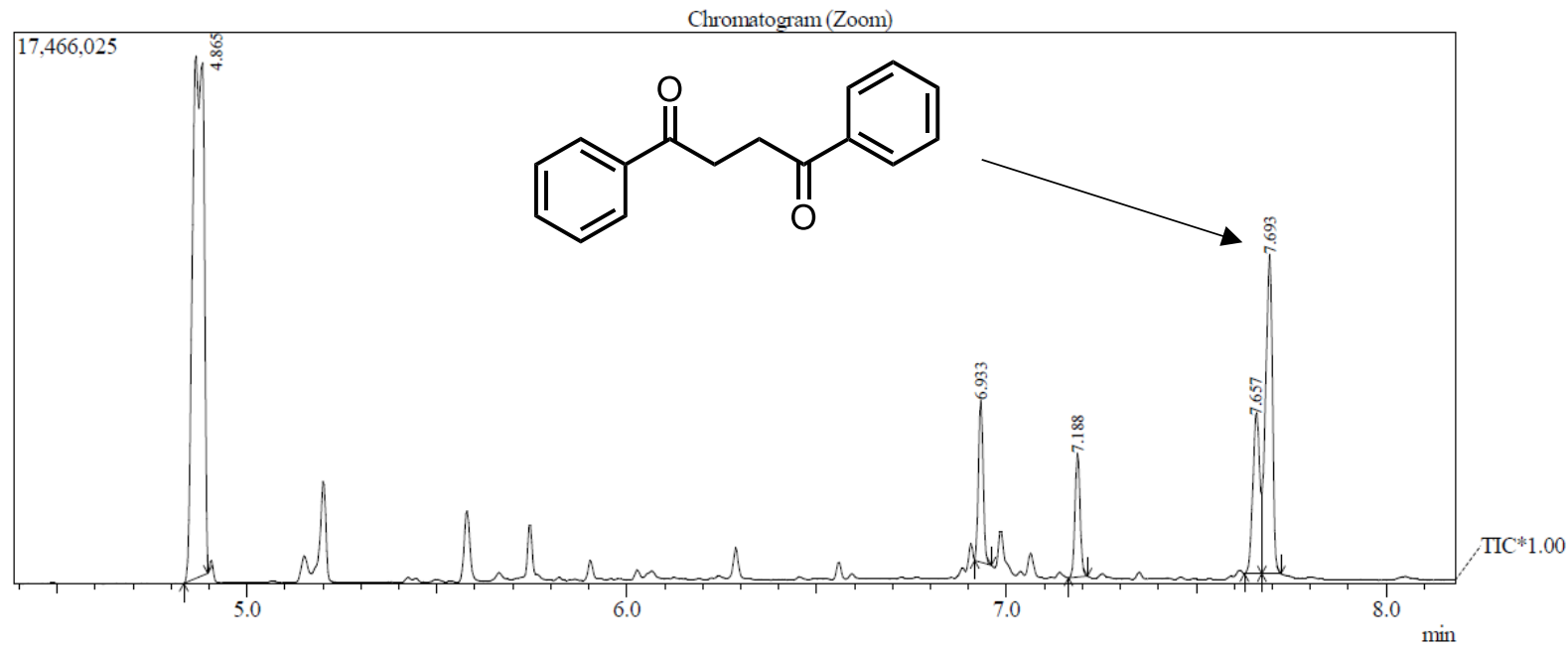

Line\#:1 R.Time:7.690(Scan\#:1165)

MassPeaks:541

RawMode:Single 7.690(1165) BasePeak:105.10(4550345)

BG Mode:None Group 1 - Event 1 Scan

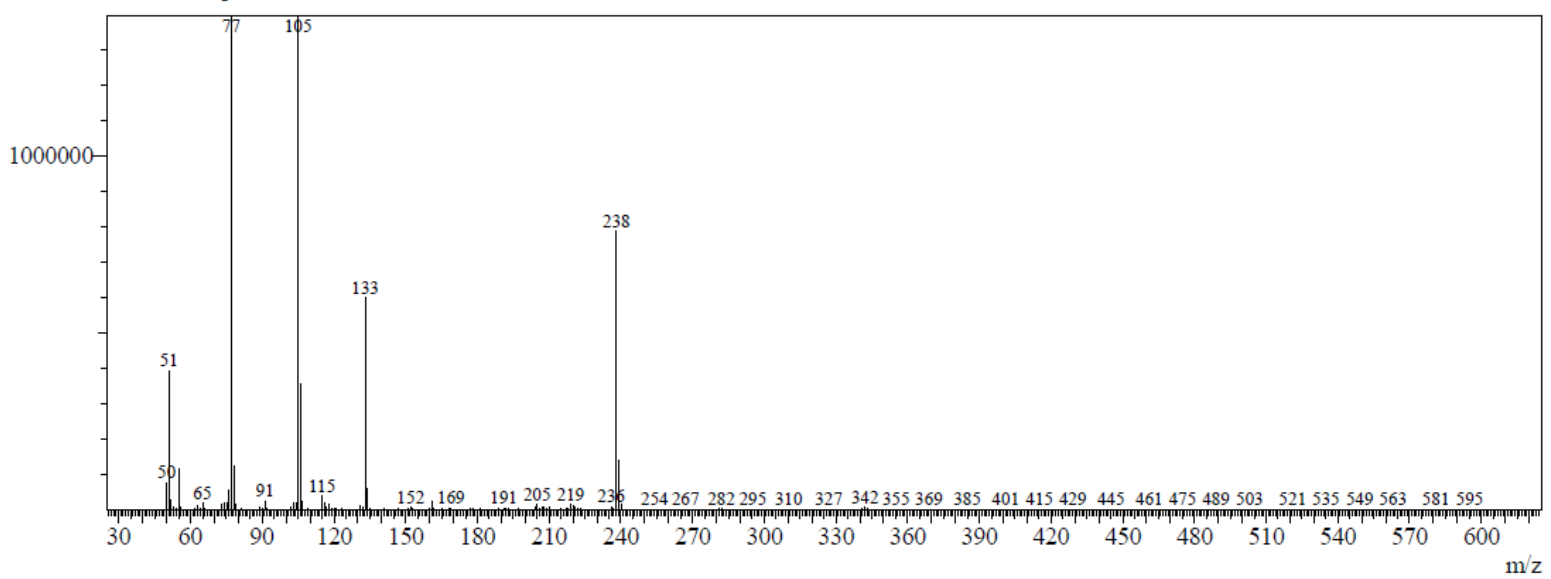

The spectra were in accordance with literature values.[20] 
[1,1'-bicyclohexane)]-2,2'-dione
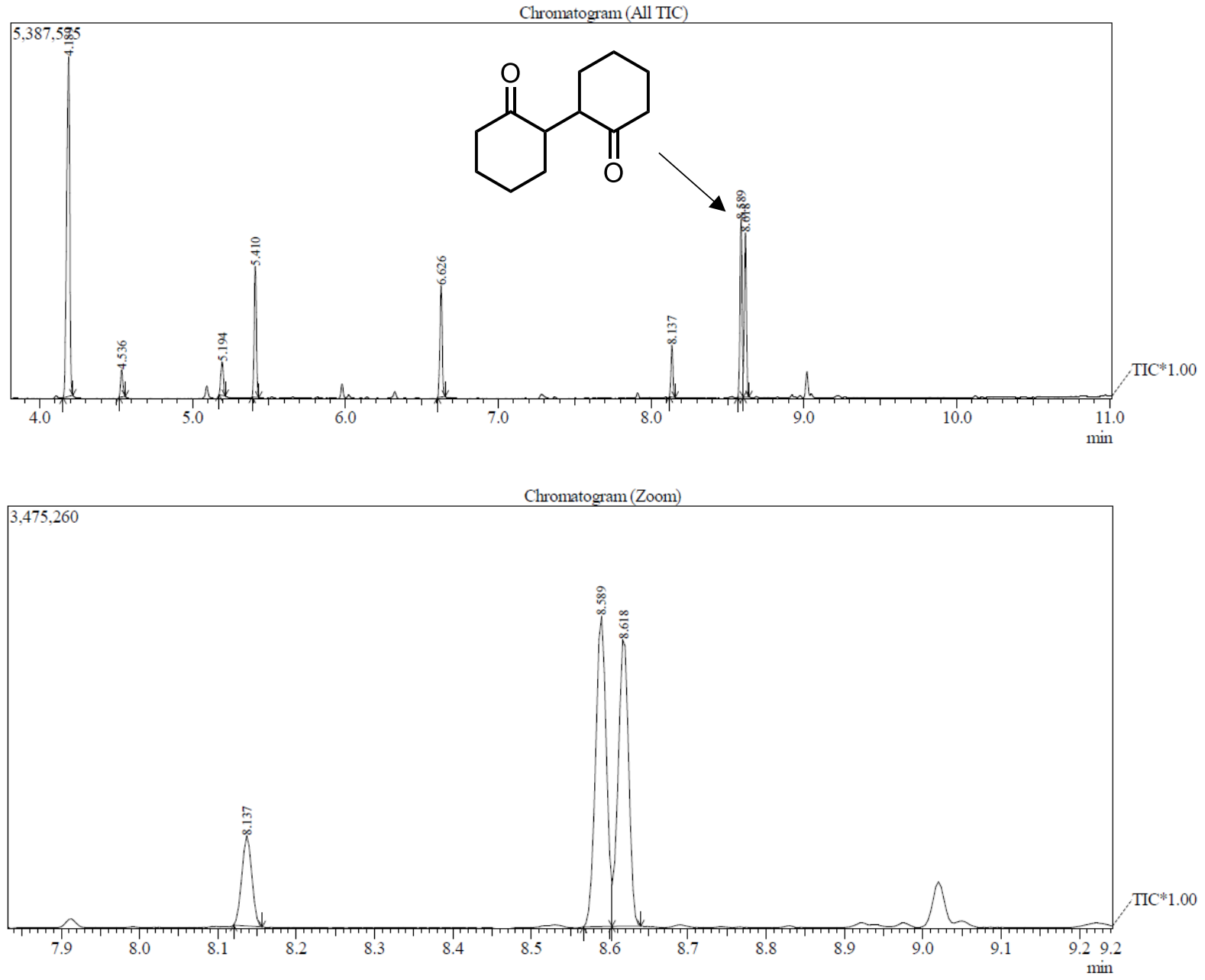

MS spectrum of the peak at $8.589 \mathrm{~min}$ :

Line\#:1 R. Time:8.590(Scan\#:1435)

MassPeaks:505

RawMode:Single 8.590(1435) BasePeak:98.10(480588)

BG Mode:None Group 1 - Event 1 Scan

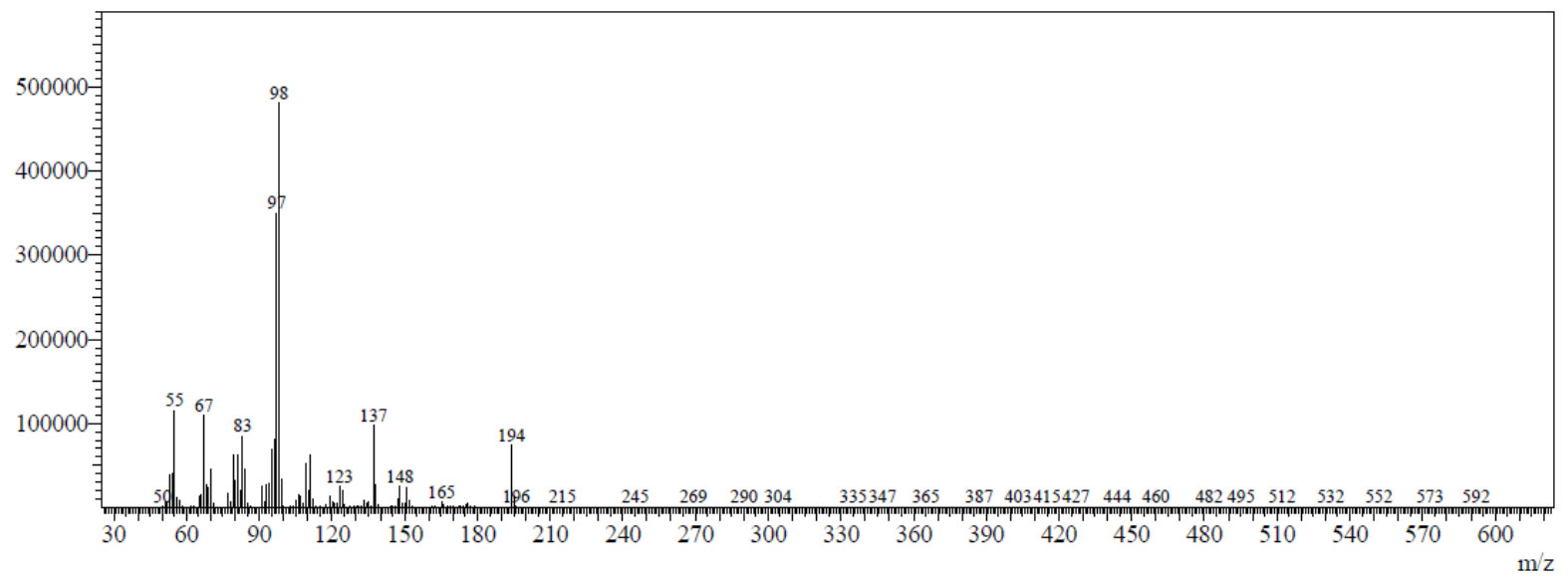

S30 


\section{MS spectrum of the peak at $8.618 \mathrm{~min}$ :}

Line\#:1 R. Time:8.620(Scan\#:1444)

MassPeaks:483

RawMode:Single 8.620(1444) BasePeak:98.10(416439)

BG Mode:None Group 1 - Event 1 Scan

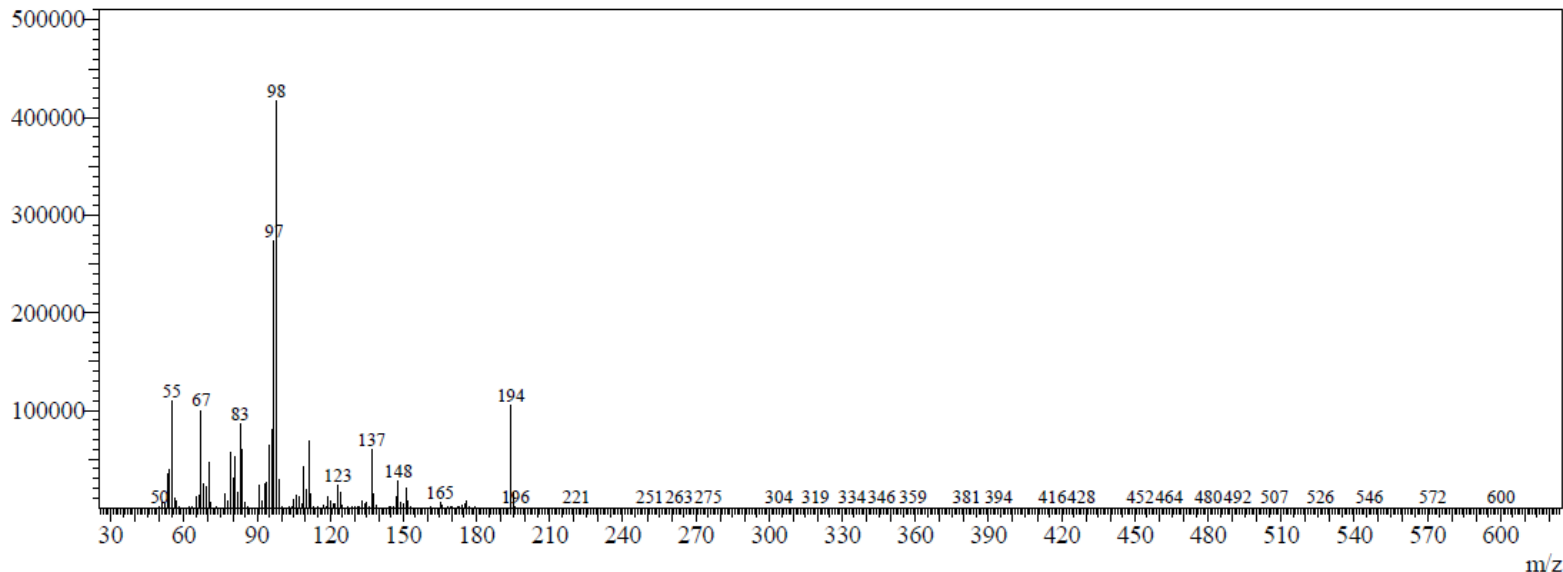

The spectra were in accordance with literature values. ${ }^{[21]}$

\section{tetradecane-6,9-dione}

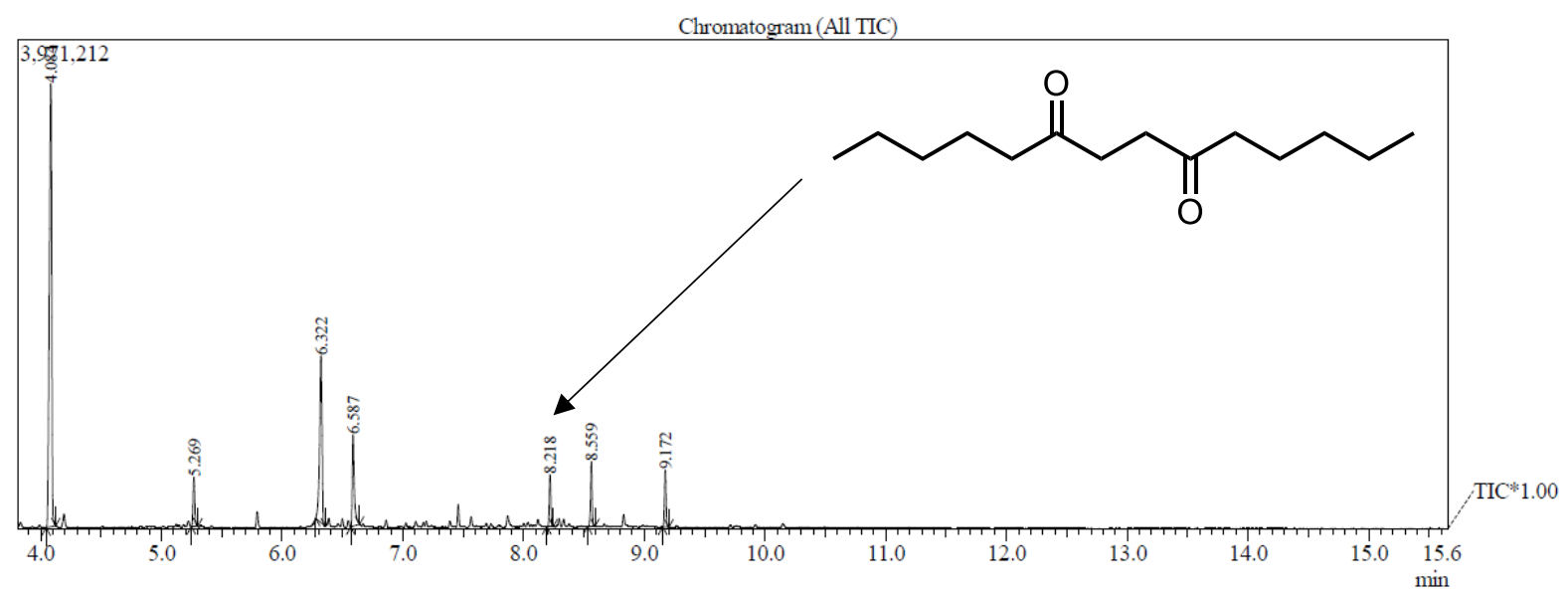

Line\#:1 R.Time:8.217(Scan\#:1323)

MassPeaks:504

RawMode:Single 8.217(1323) BasePeak:99.10(63818)

BG Mode:None Group 1 - Event 1 Scan

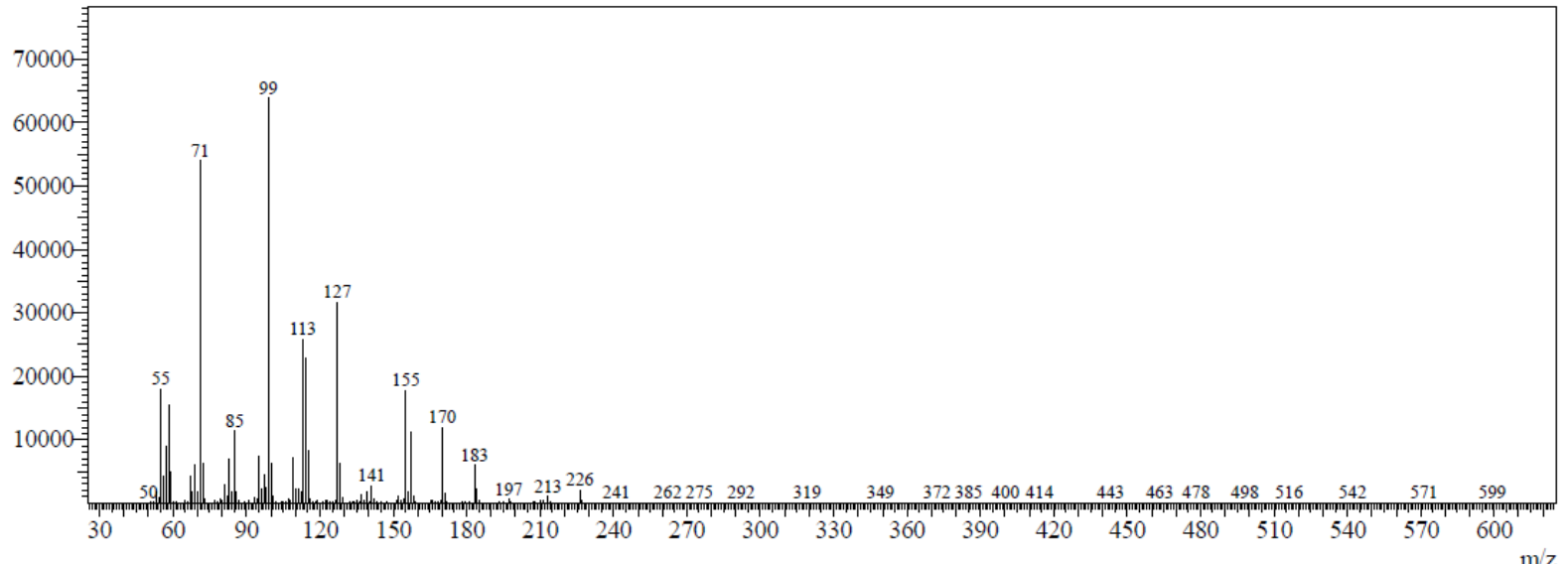


1,4-bis(4-methoxyphenyl)butane-1,4-dione (+ impurities)

${ }^{1}$ H NMR $\left(300 \mathrm{MHz}, \mathrm{CDCl}_{3}\right): \delta=8.11-8.00(\mathrm{~m}, 2 \mathrm{H}), 7.03-7.6 .93(\mathrm{~m}, 2 \mathrm{H}), 3.91(\mathrm{~s}, 3 \mathrm{H}), 3.43(\mathrm{~s}$, $3 \mathrm{H}) \mathrm{ppm}$.
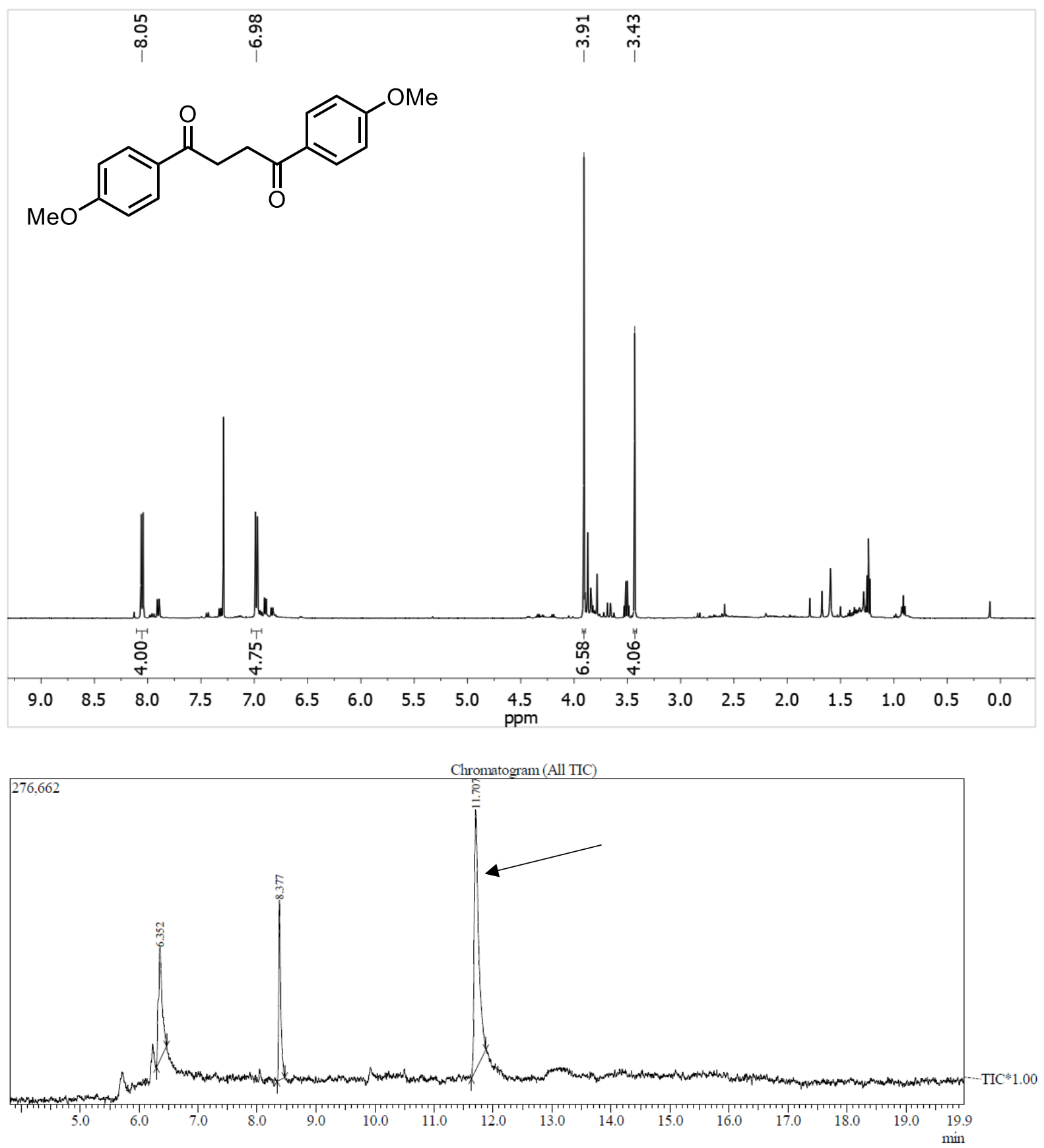
Line\#:1 R.Time:11.740(Scan\#:2380)

MassPeaks:520

RawMode:Single 11.740(2380) BasePeak:135.15(79253)

BG Mode:None Group 1 - Event 1 Scan

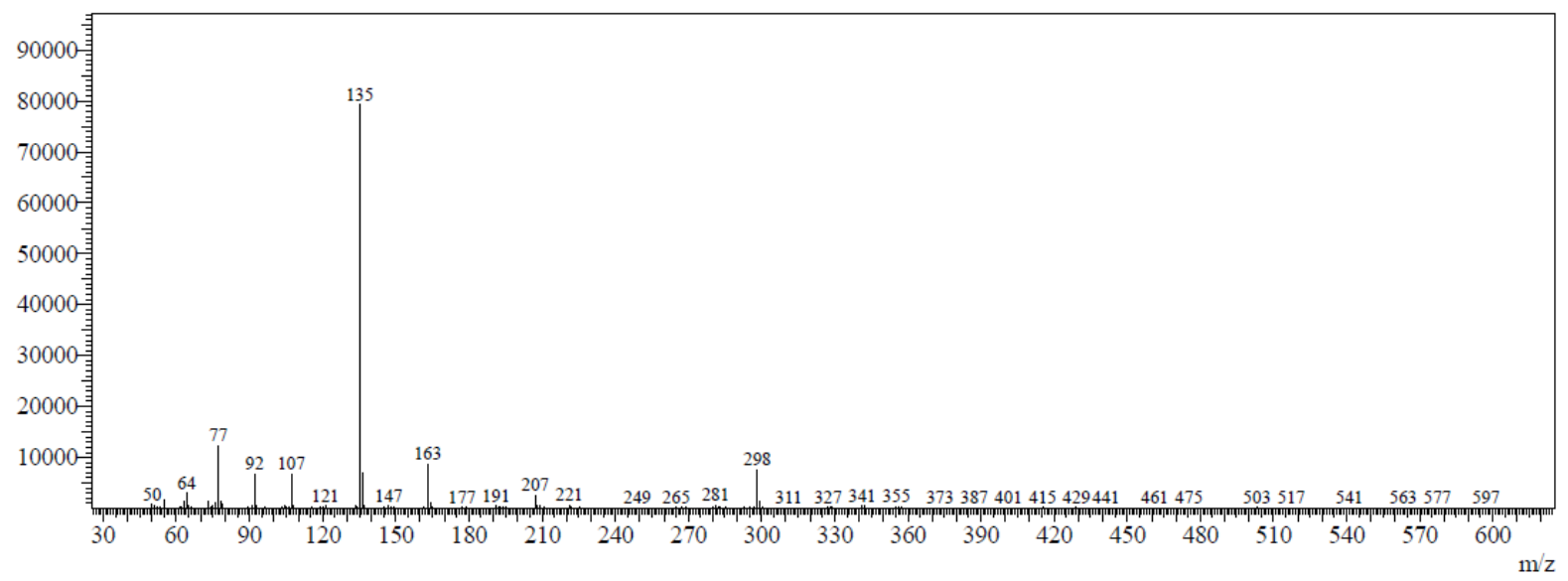

The data were in accordance with literature values. ${ }^{[22,23]}$ 
12. NMR spectra of the synthesized compounds

\section{2-(trimethylsiloxy)propene}
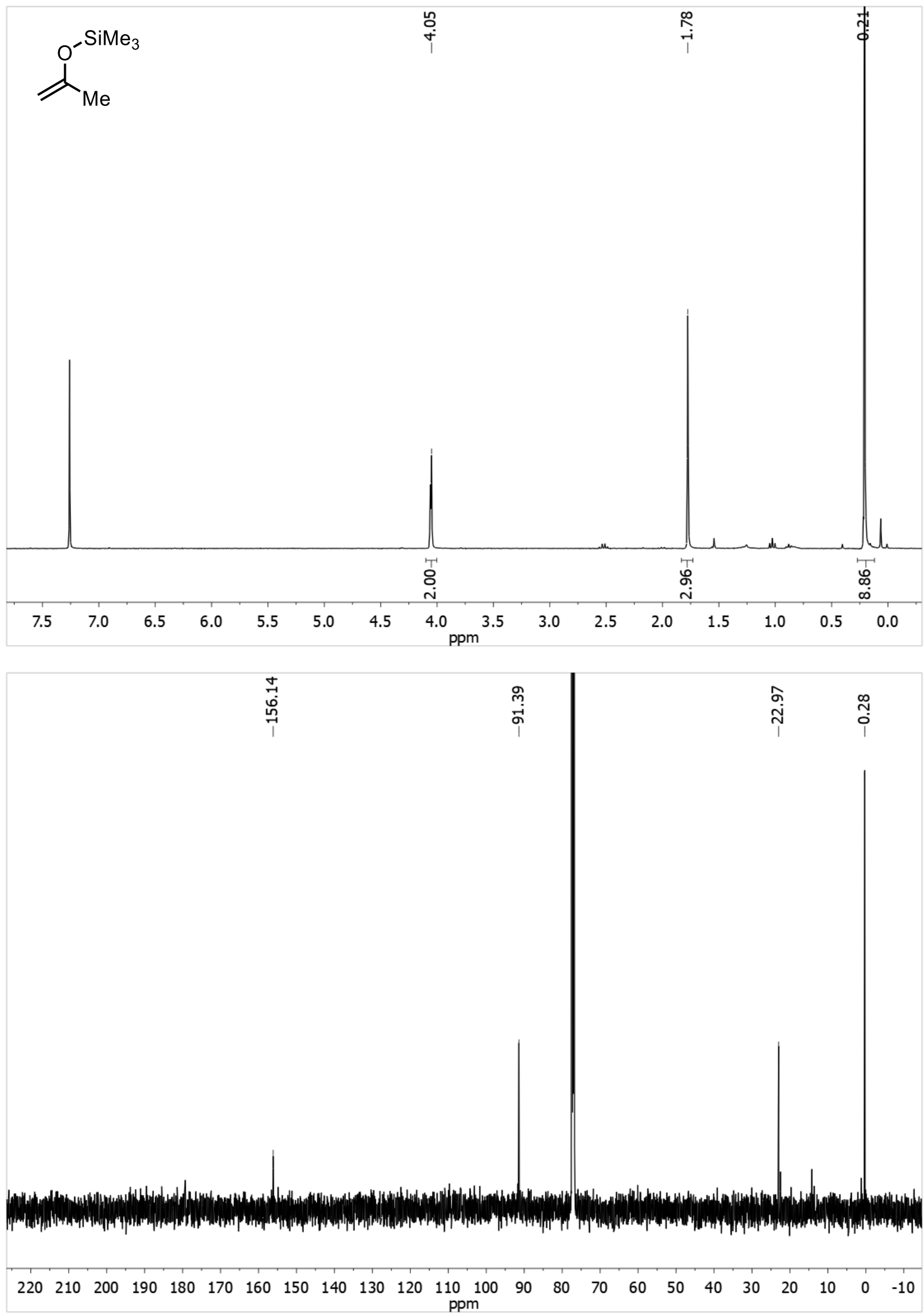
3,3-dimethyl-2-trimethylsiloxy-1-butene
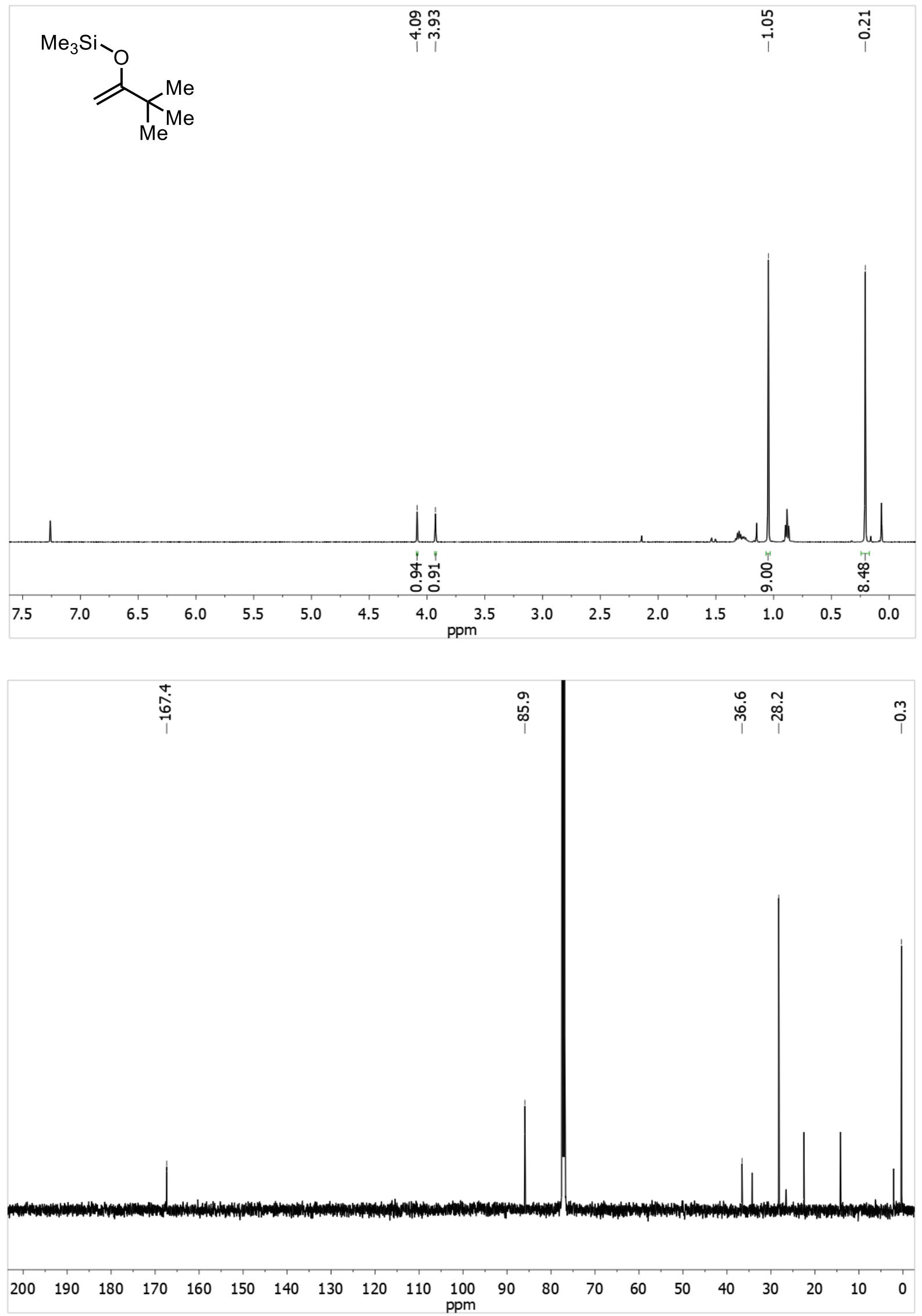
1-trimethylsiloxycyclohexene
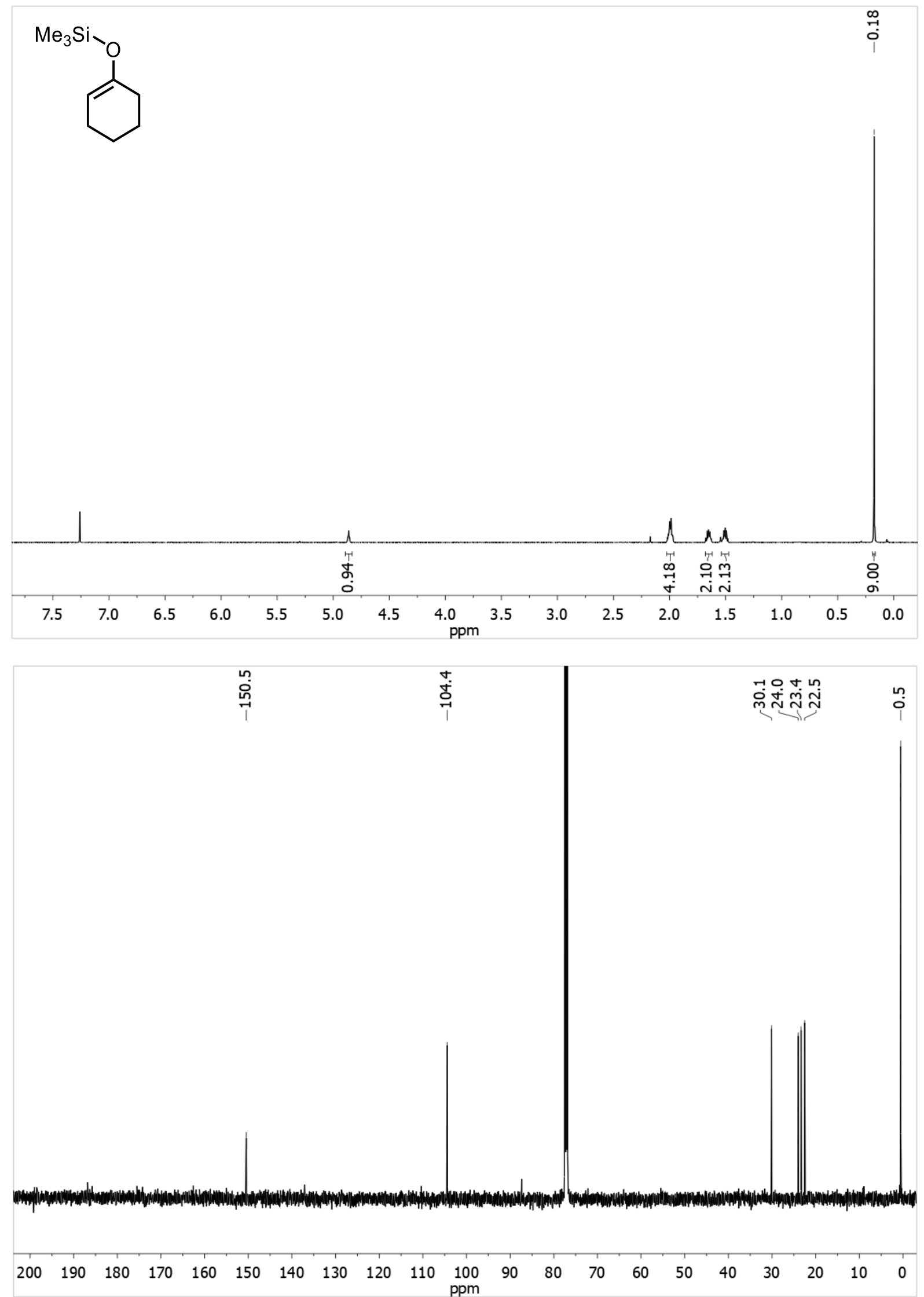


\section{2-trimethylsiloxy-1-heptene}

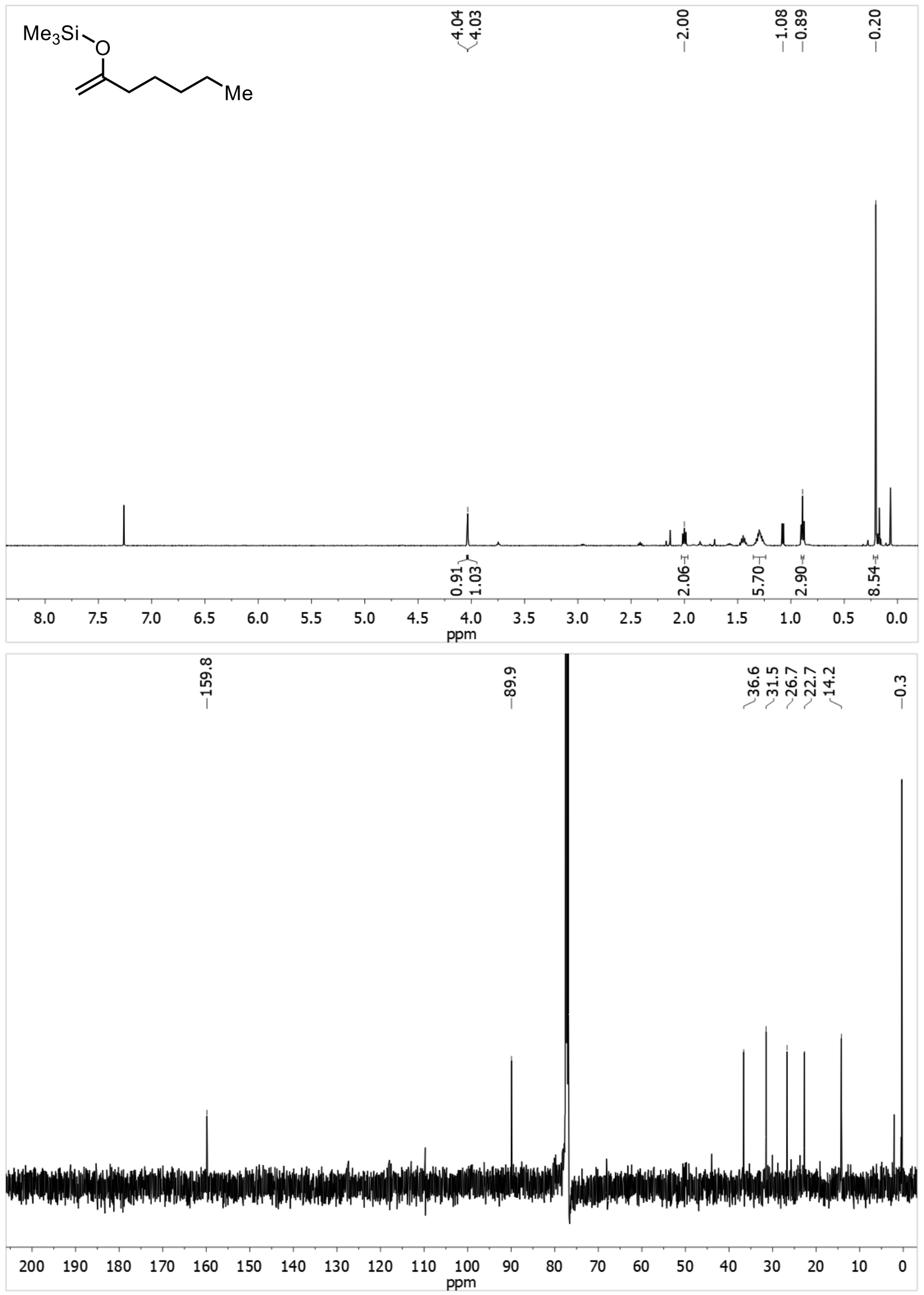


$\alpha$-trimethylsiloxystyrene
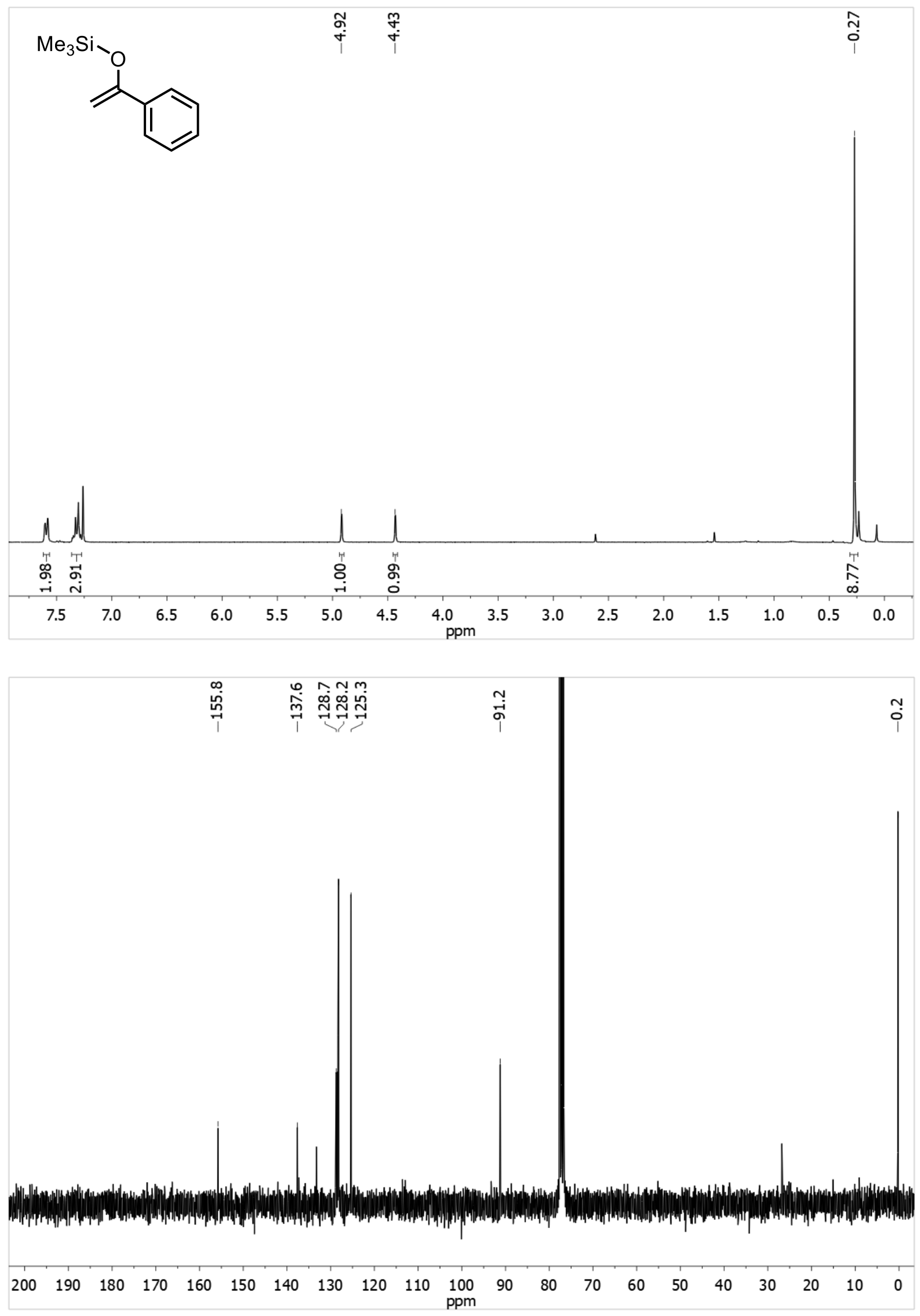

S38 
$\alpha$-trimethylsiloxy-4-nitrostyrene
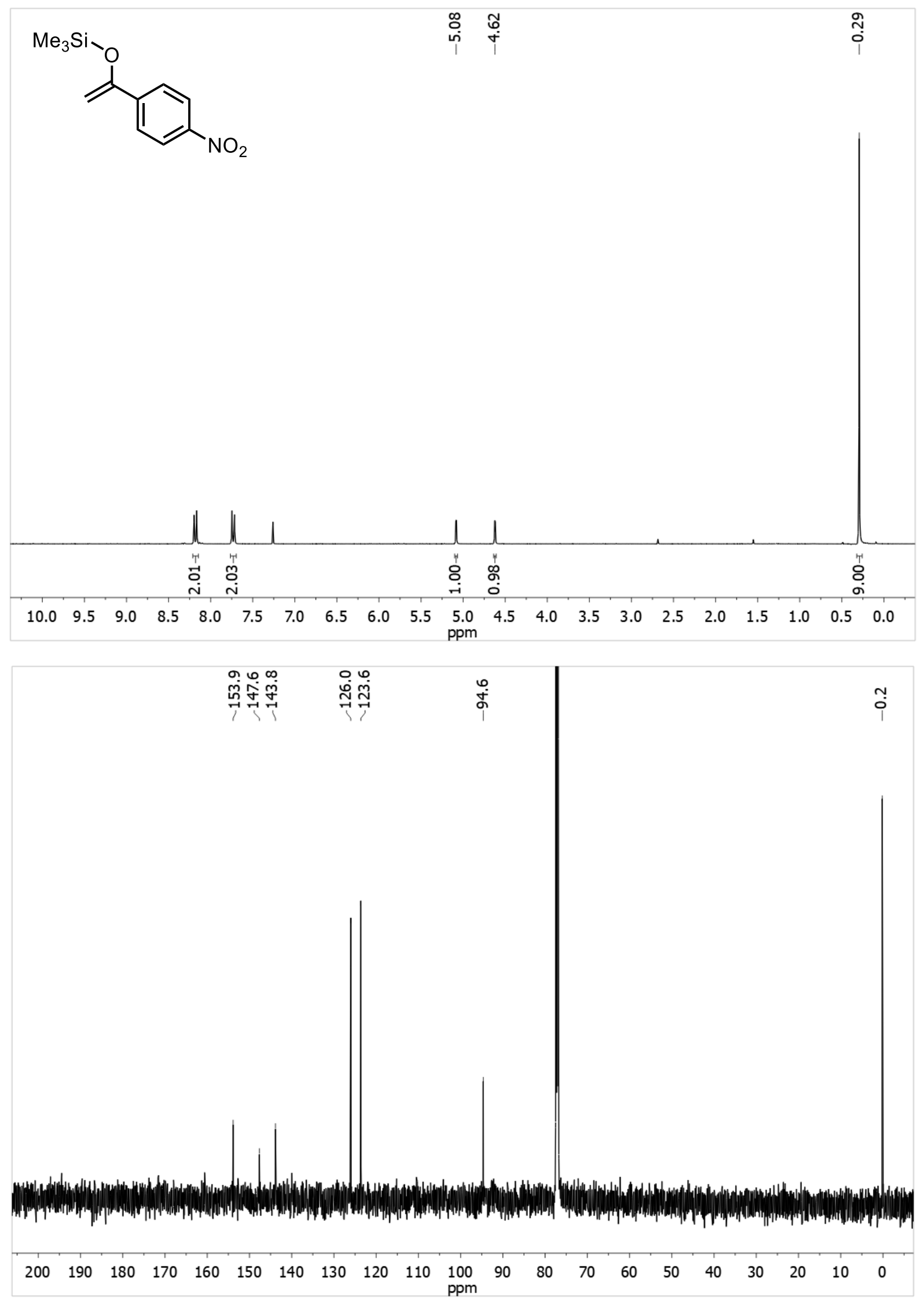

S39 


\section{4-methoxy- $\alpha$-trimethylsiloxystyrene}
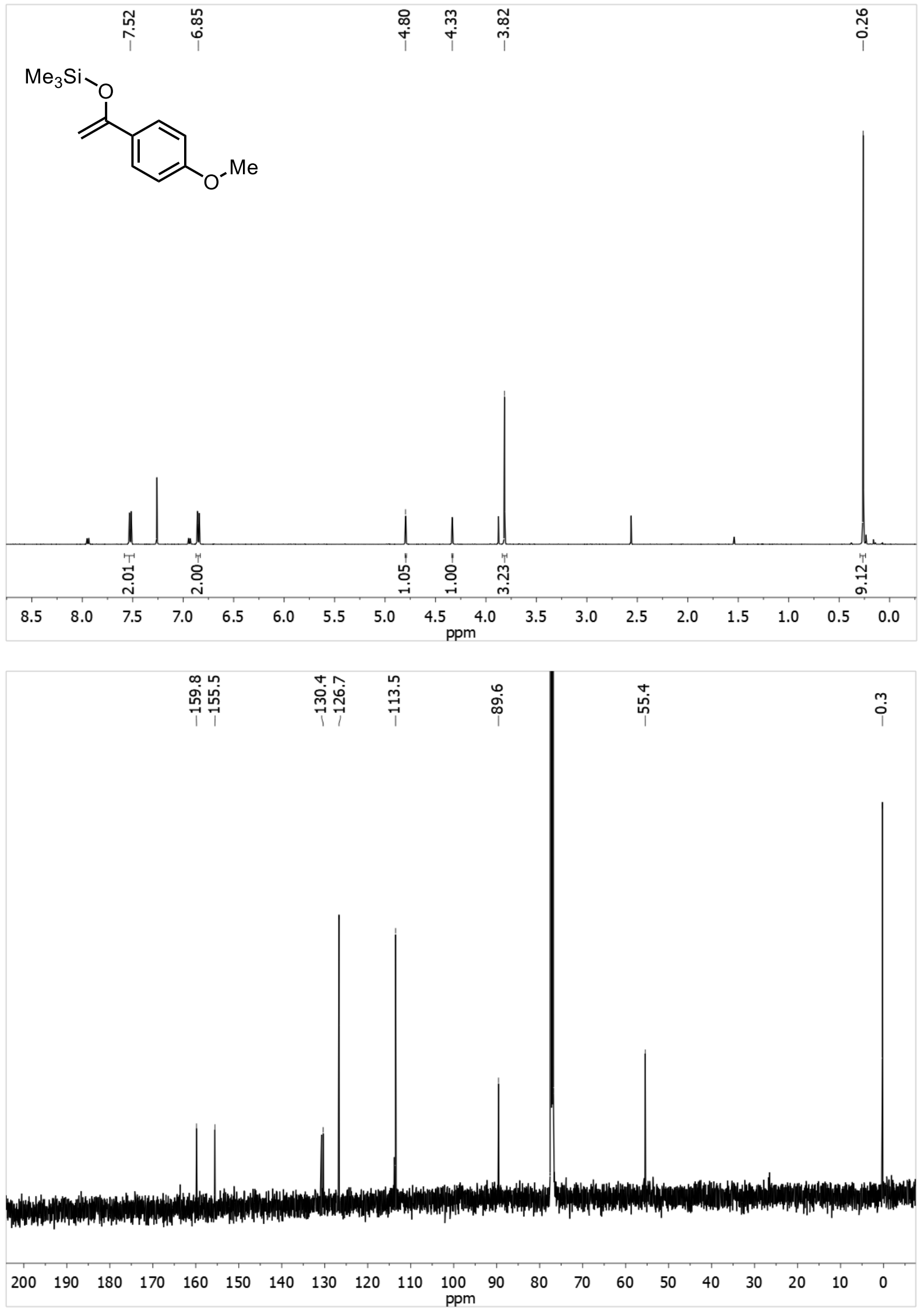
ethyl 2-oxo-1-(2-oxopropyl)cyclopentane-1-carboxylate (2a)
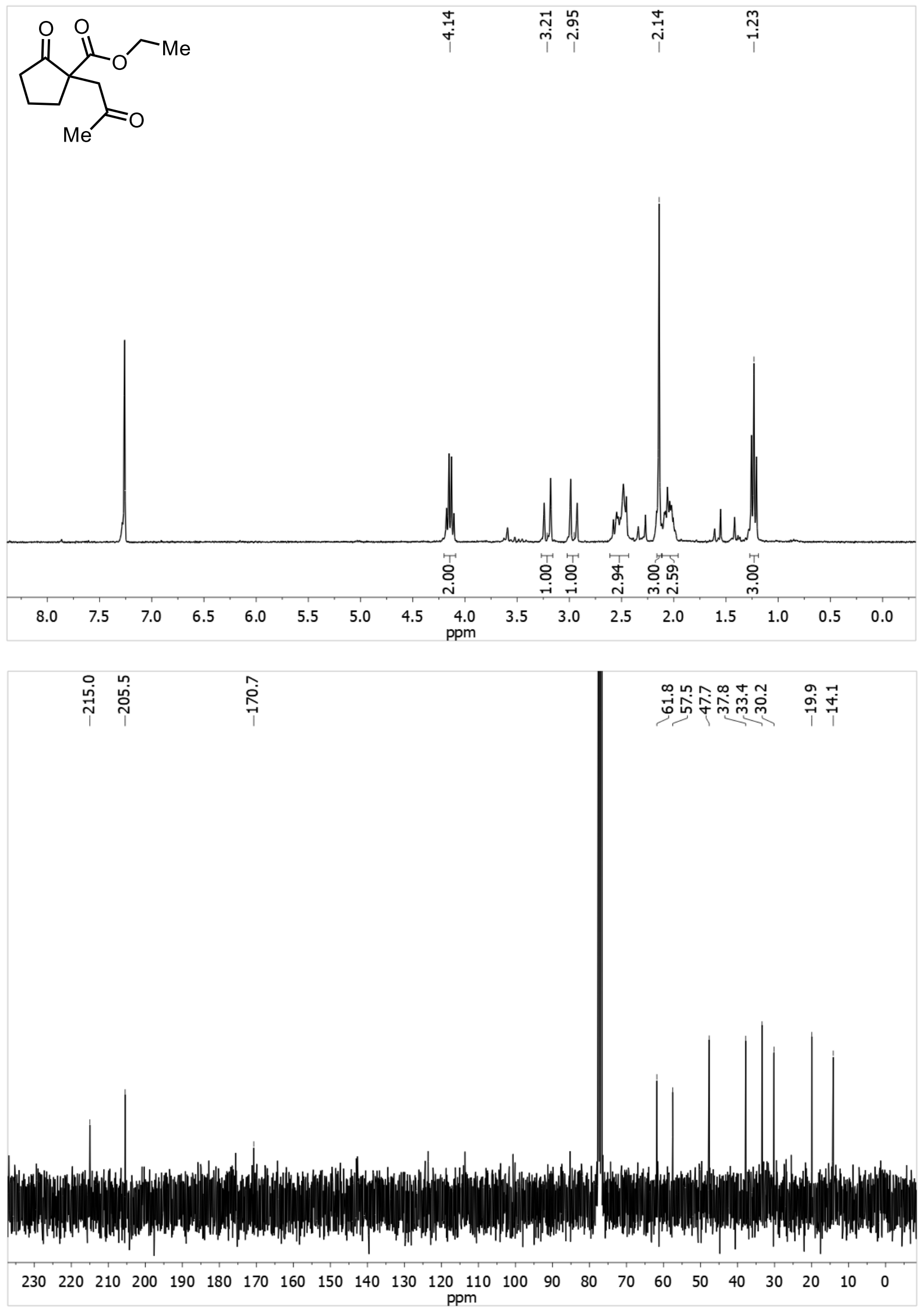
isopropyl-2-oxo-1-(2-oxopropyl)cyclopentane-1-carboxylate (2b):
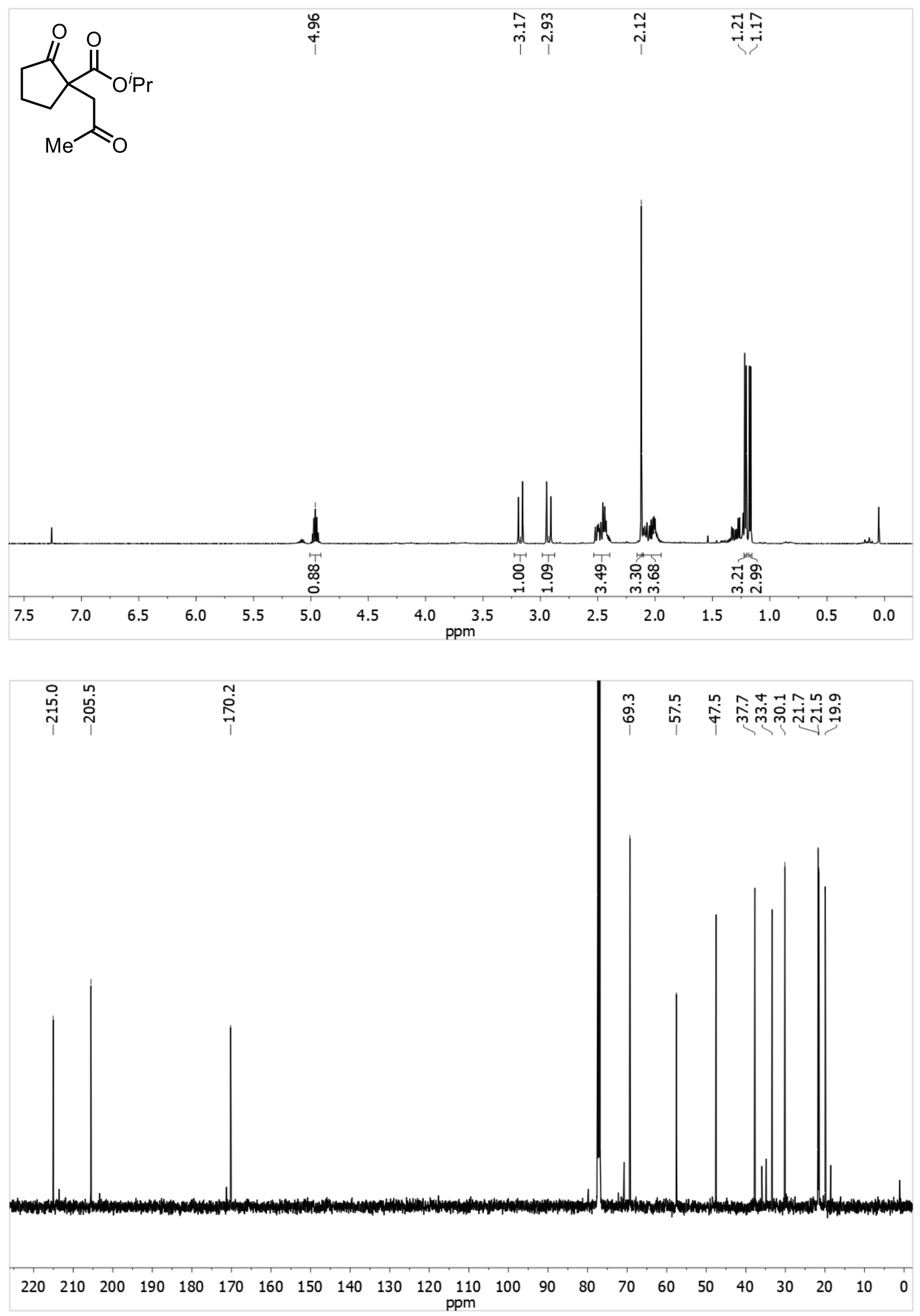

S42 
ethyl 2-oxo-1-(2-oxopropyl)cyclohexane-1-carboxylate (2e)
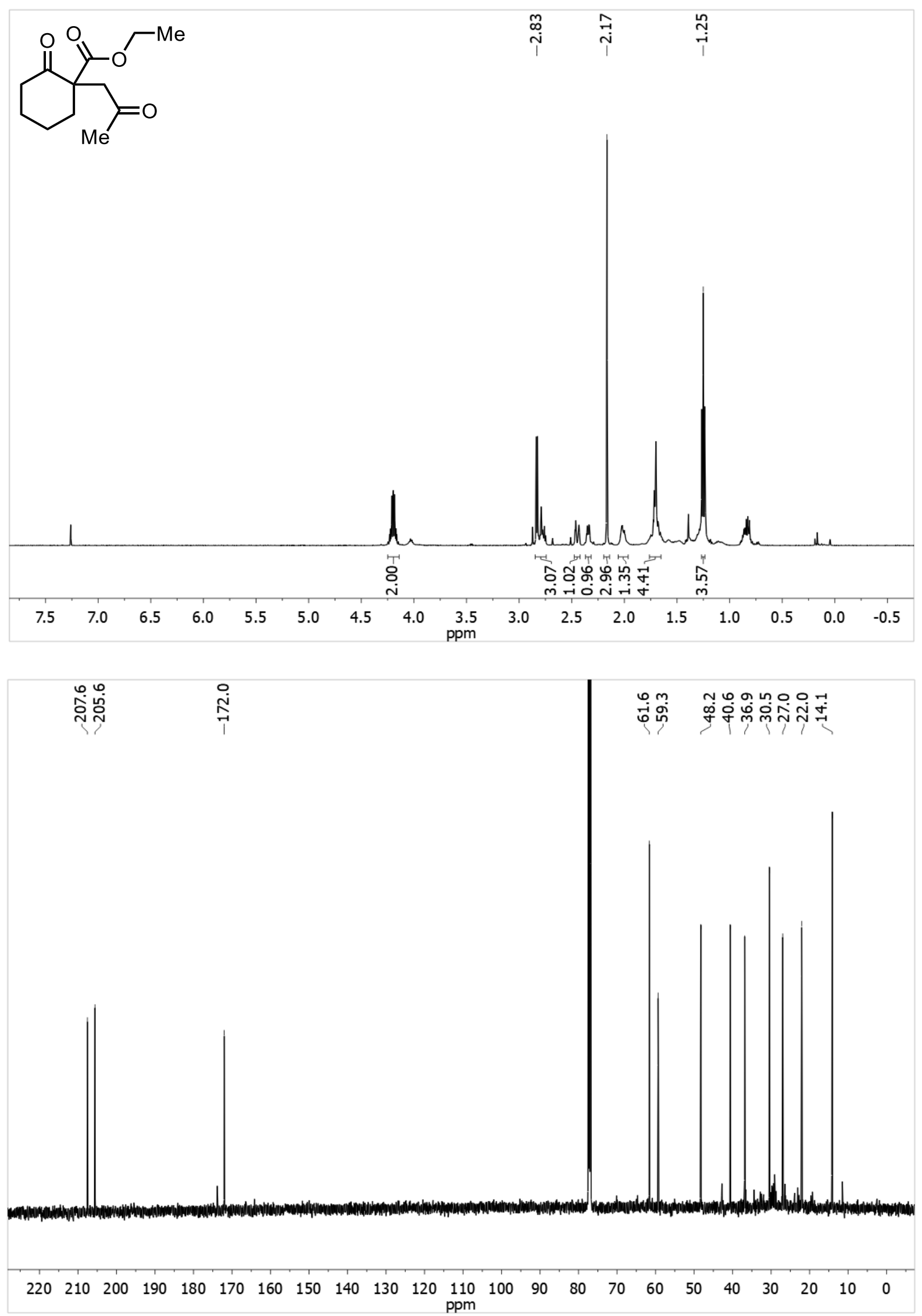
methyl 2-oxo-1-(2-oxopropyl)cycloheptane-1-carboxylate (2f)
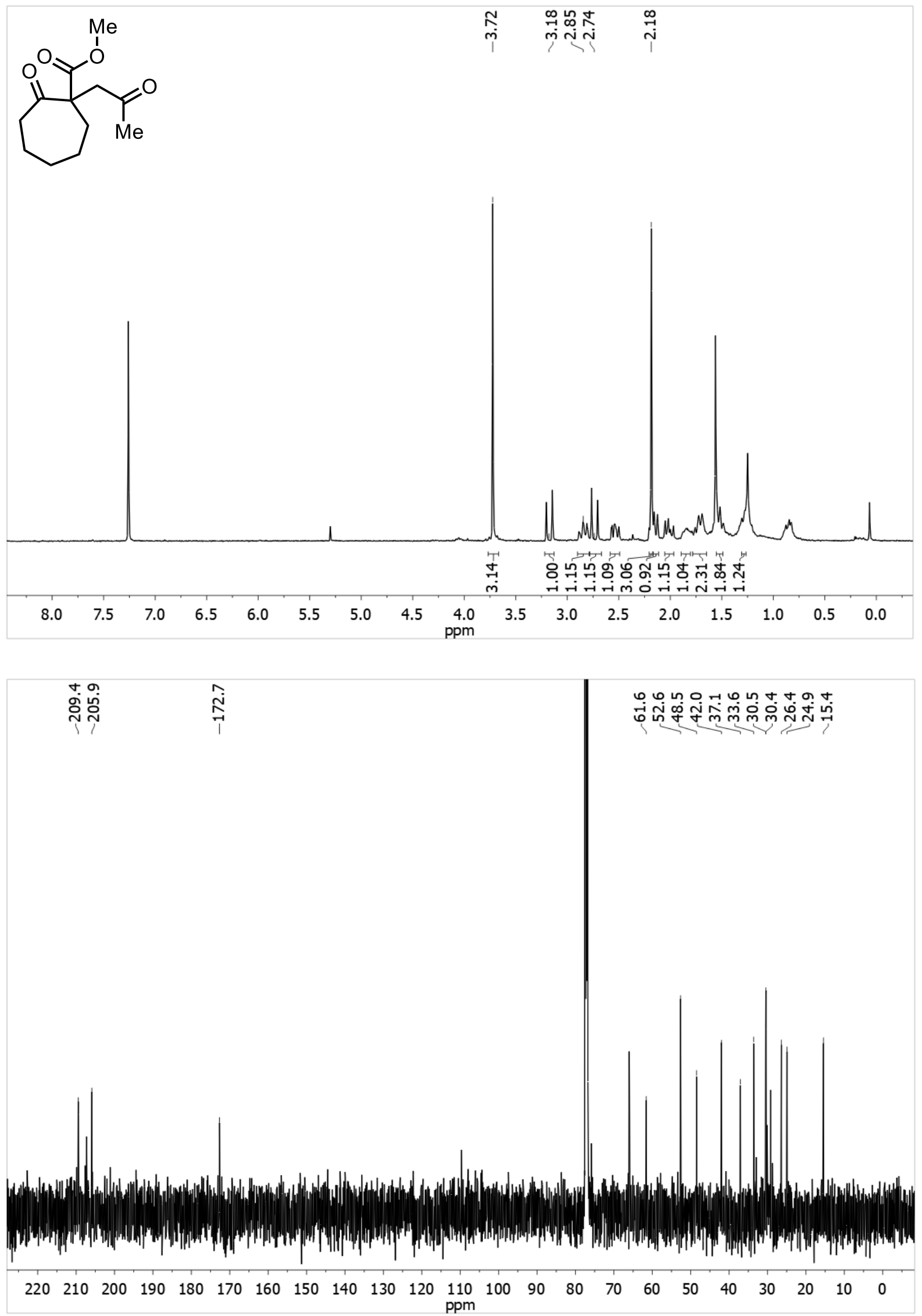
2-acetyl-2-(2-oxopropyl)cyclopentane-1-one (2g):

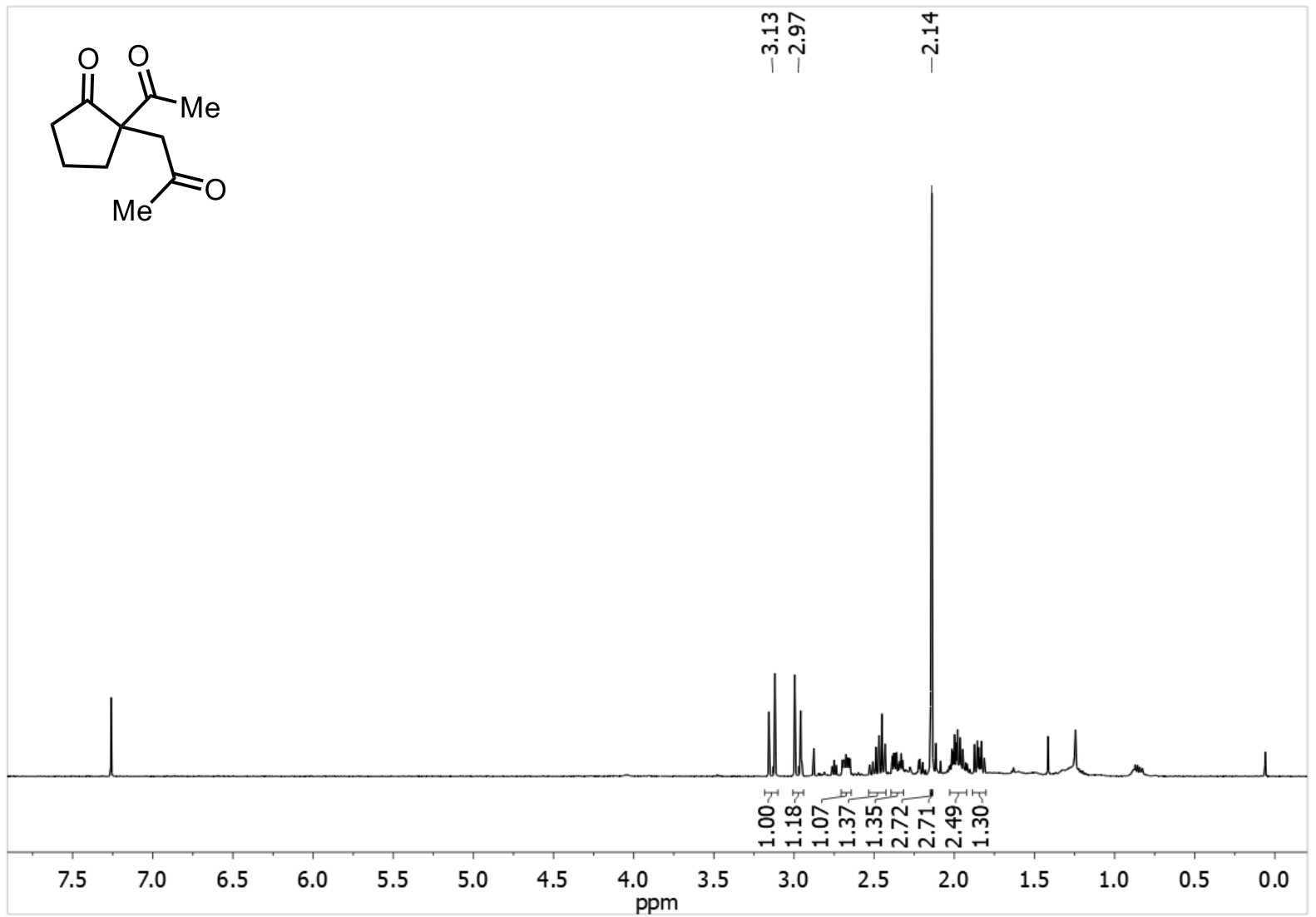

กำกั่

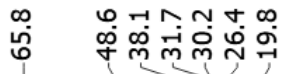

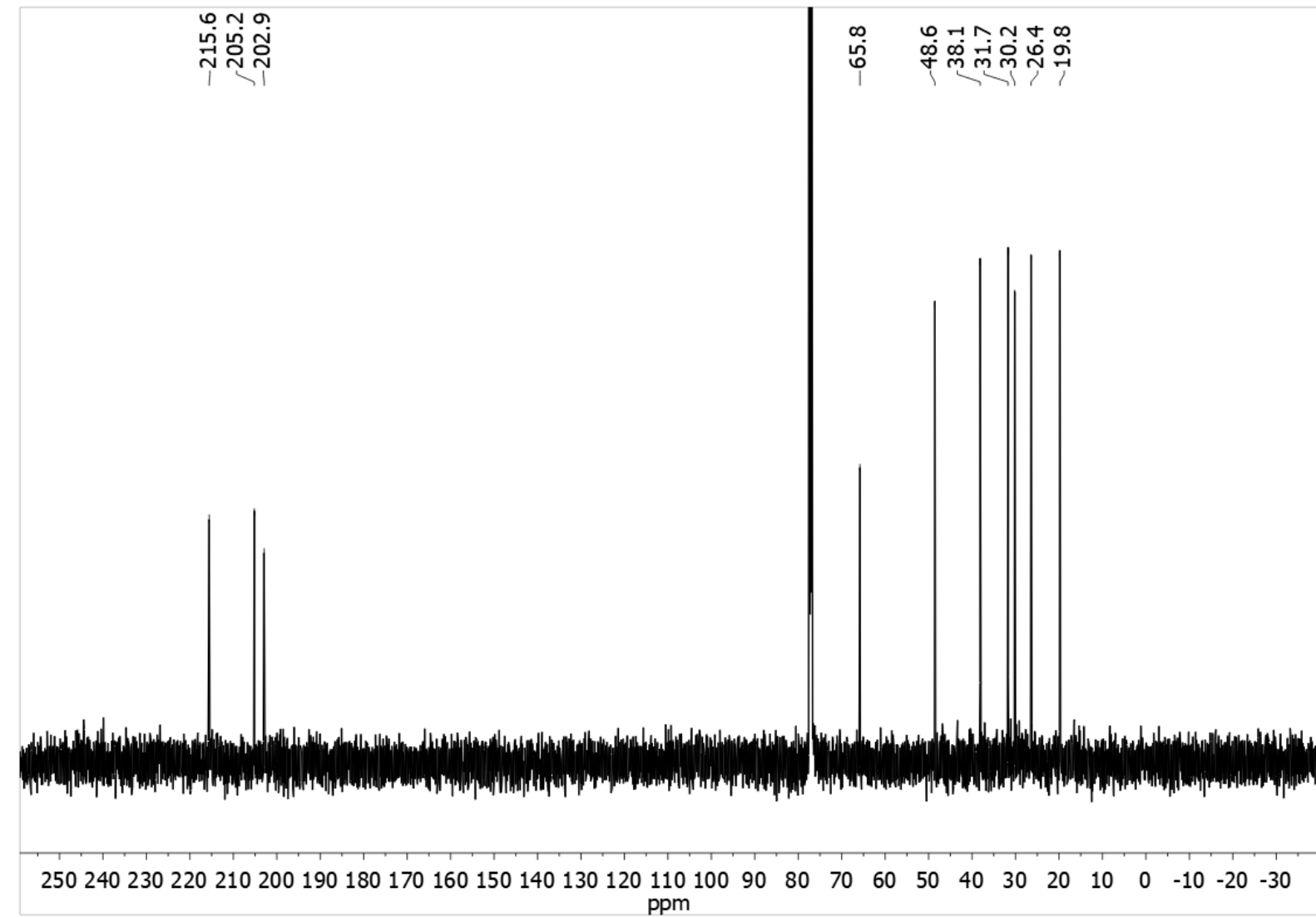


methyl 1-oxo-2-(2-propyl)-2,3-dihydro-1 $H$-indene-2-carboxylate (2i)
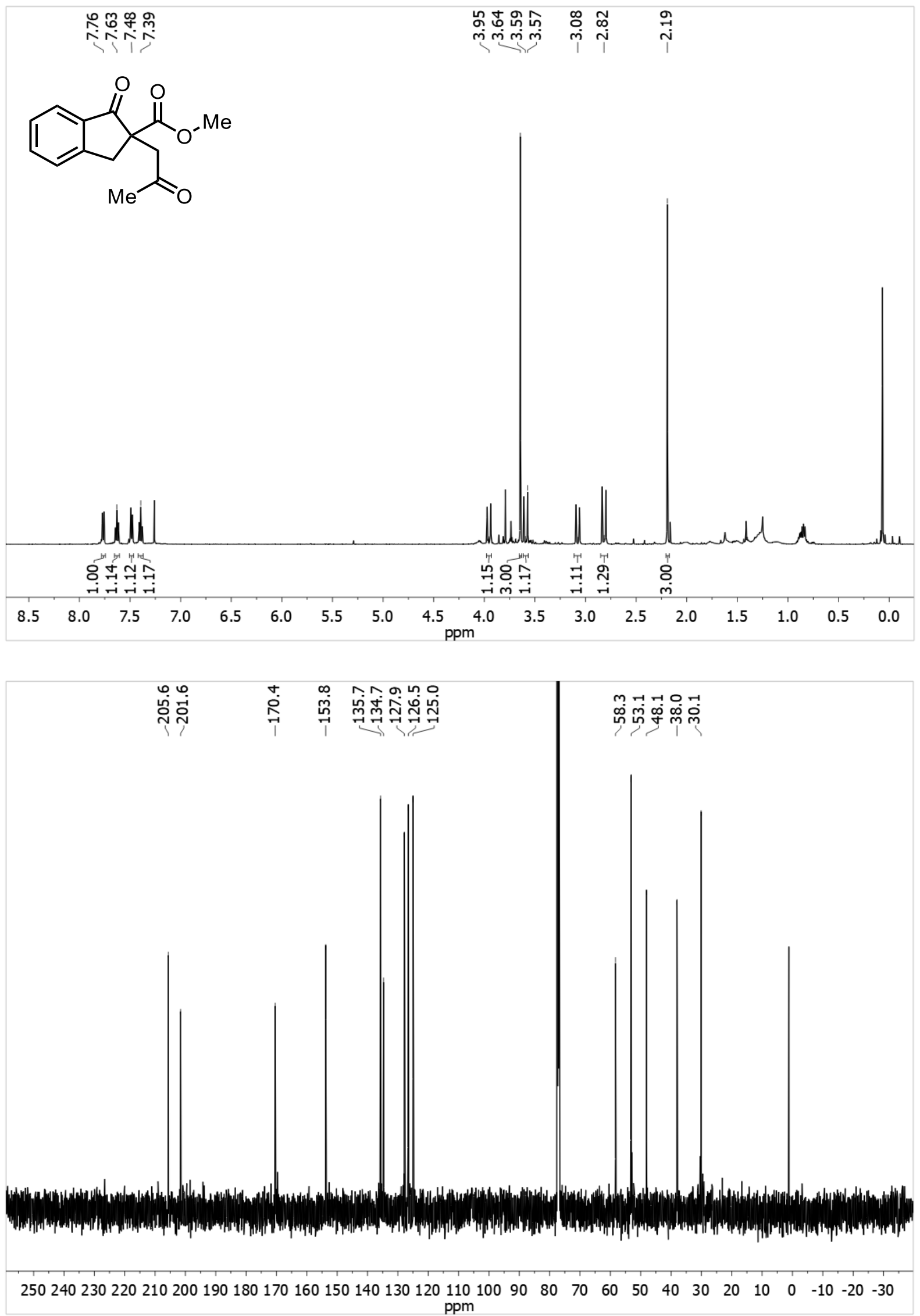
methyl 4-bromo-1-oxo-2-(2-oxopropyl)-2,3-dihydro-1 H-indene-2-carboxylate (2j)

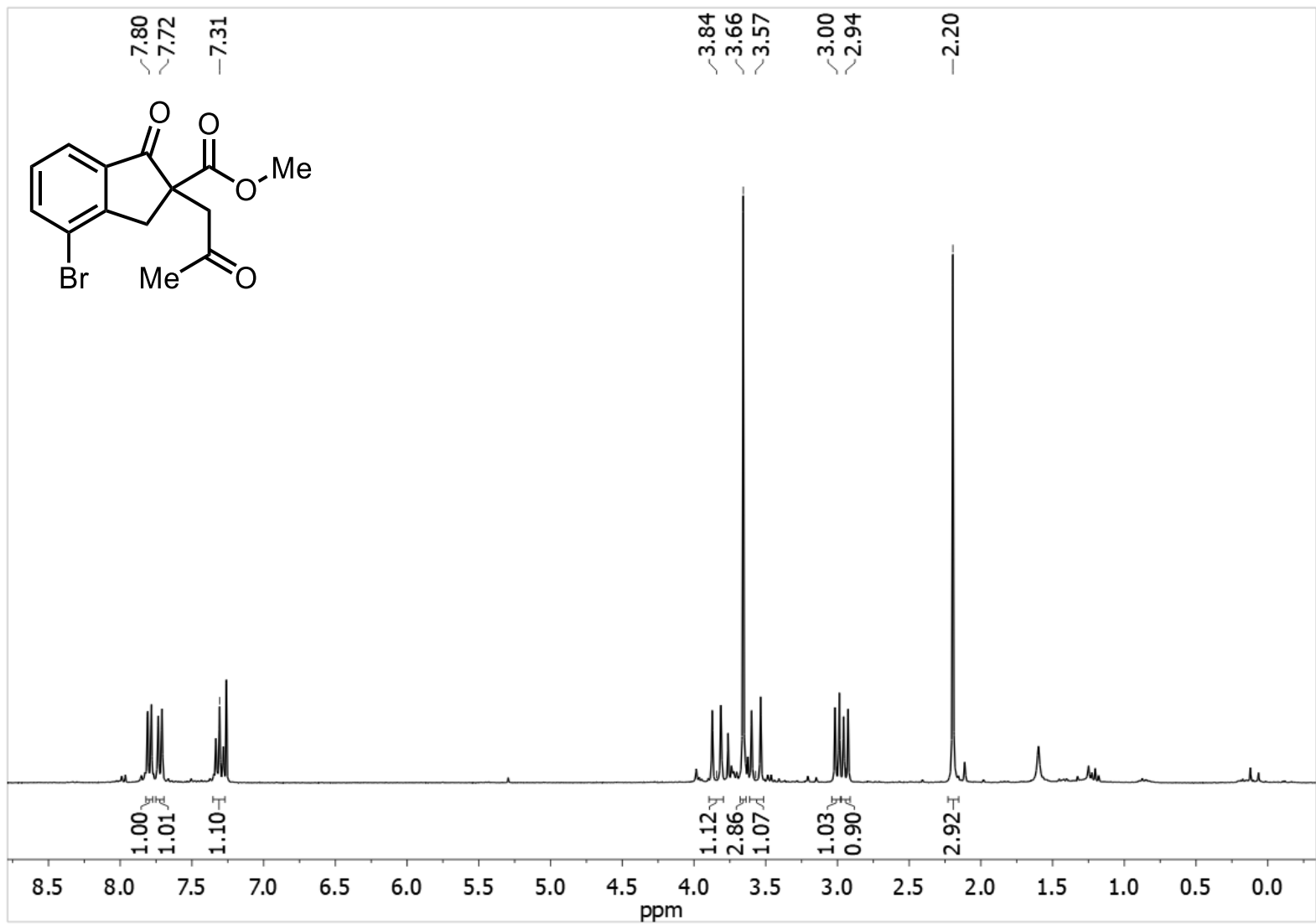

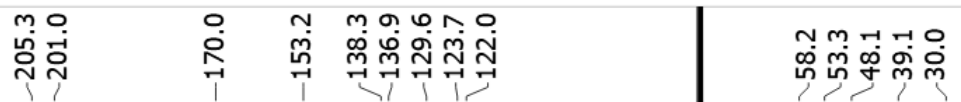

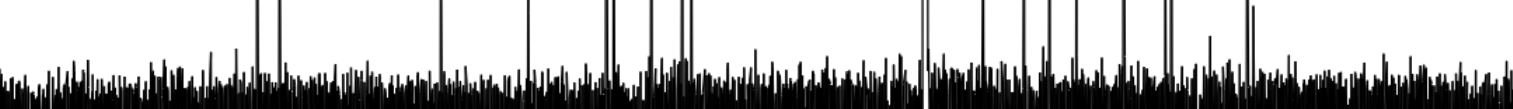

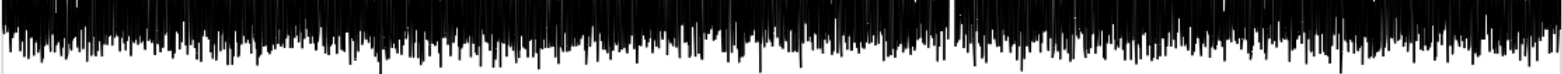

$25024023022021020019018017016015014013012011010090 \quad 80 \quad 70 \quad 60 \begin{array}{llllllllll}40 & 30 & 20 & 10 & 0 & -10 & -20 & -30\end{array}$ 
methyl 5,6-dimethoxy-1-oxo-2-(2-oxopropyl)-2,3-dihydro-1H-indene-2-carboxylate (2k)
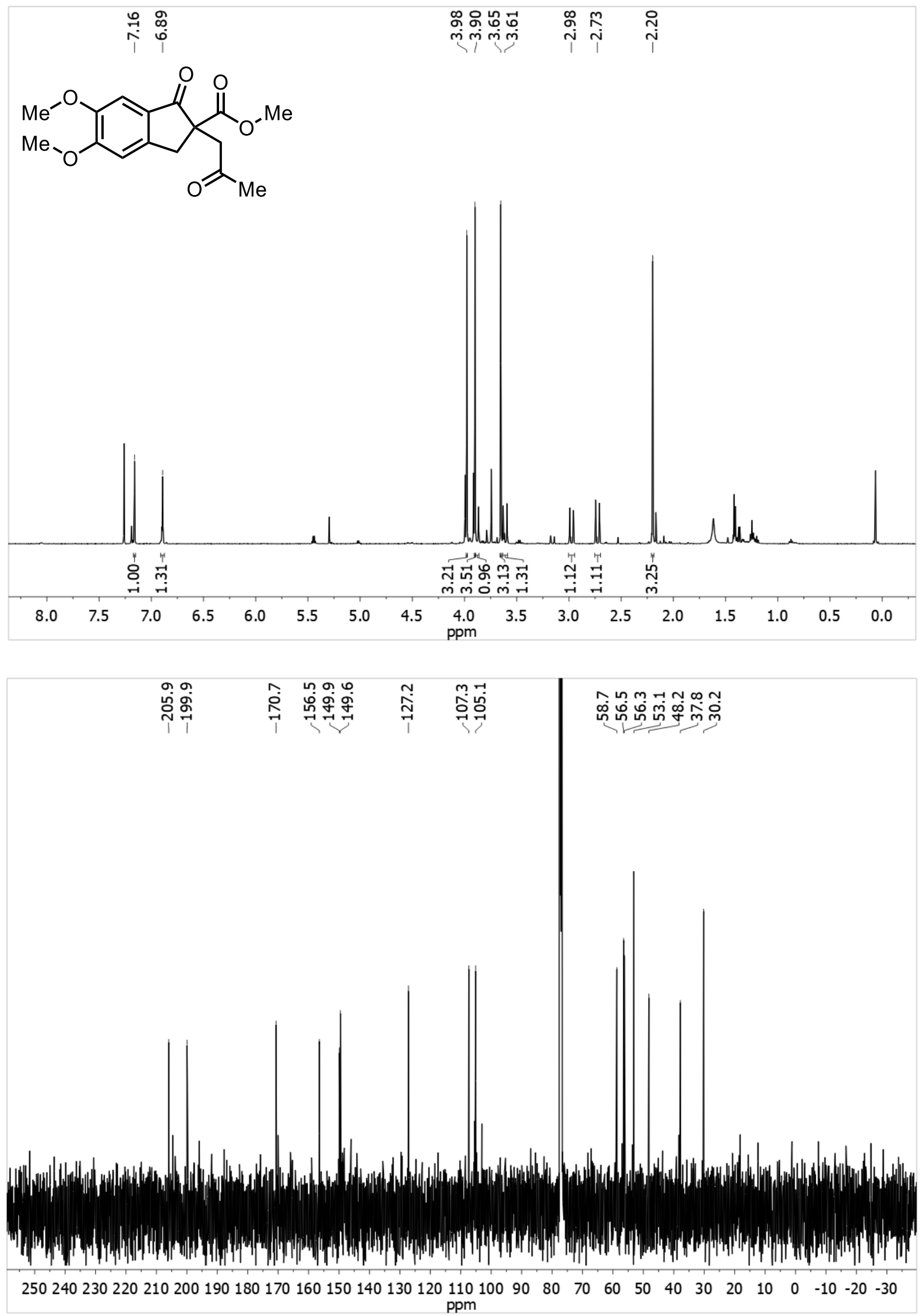
ethyl 1-oxo-2-(2-oxopropyl)-1,2,3,4-tetrahydronaphtalene-2-carboxylate (2I)
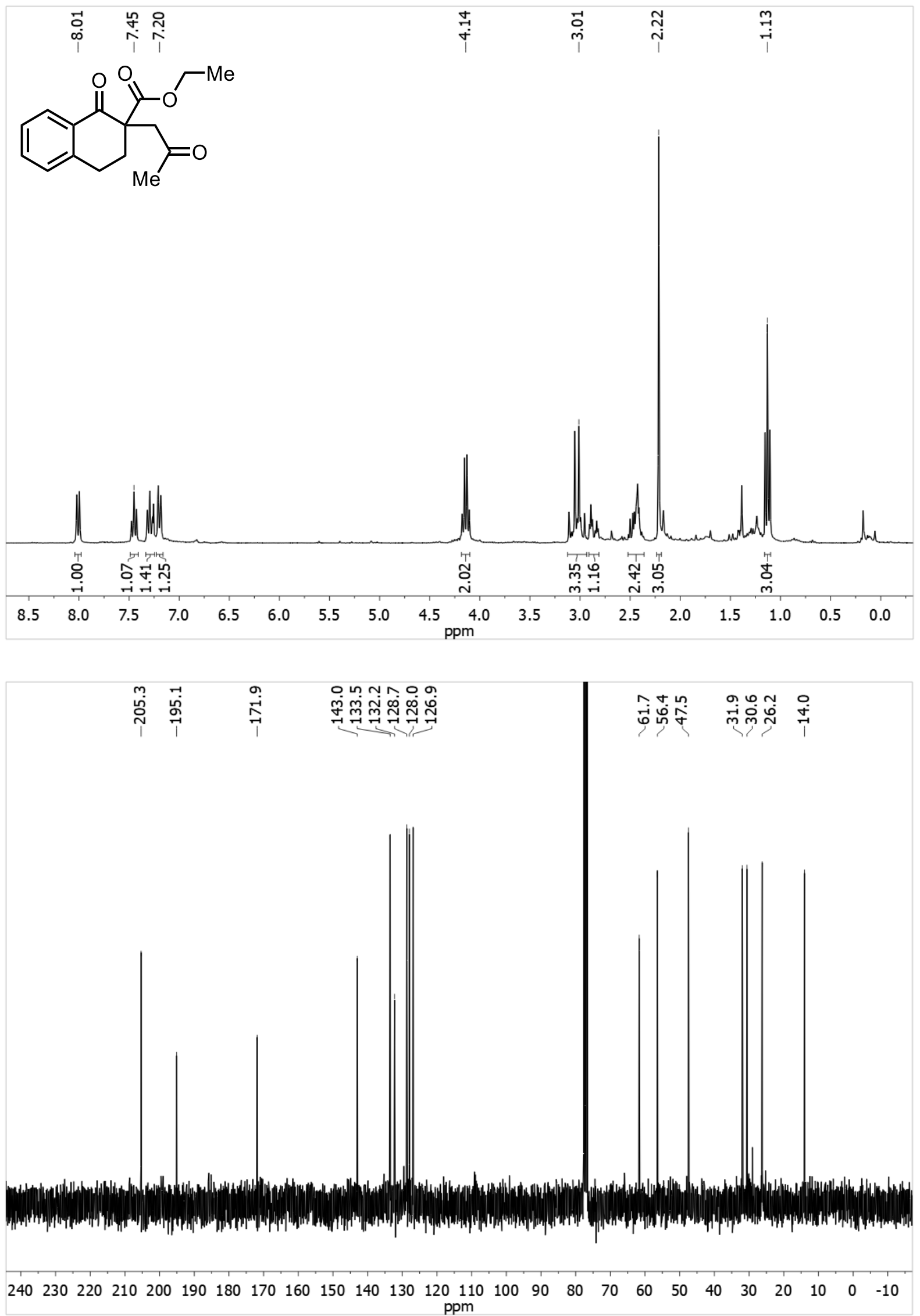
methyl 6-methoxy-1-oxo-2-(2-oxopropyl)-1,2,3,4-tetrahydronaphtalene-2-carboxylate (2m)
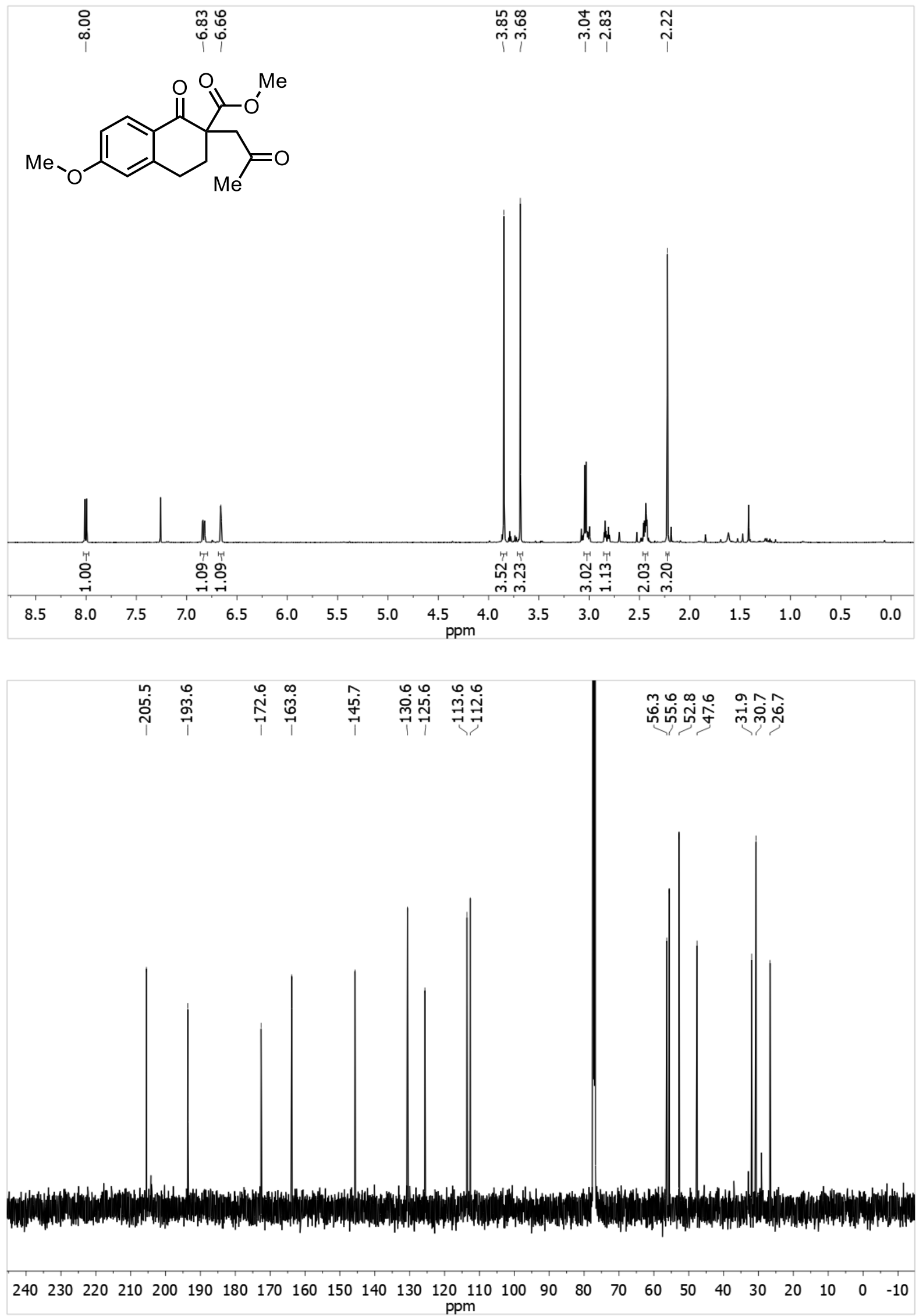
methyl-1-oxo-2-(2-oxoheptyl)-2,3-dihydro-1H-indene-2-carboxylate (2n):
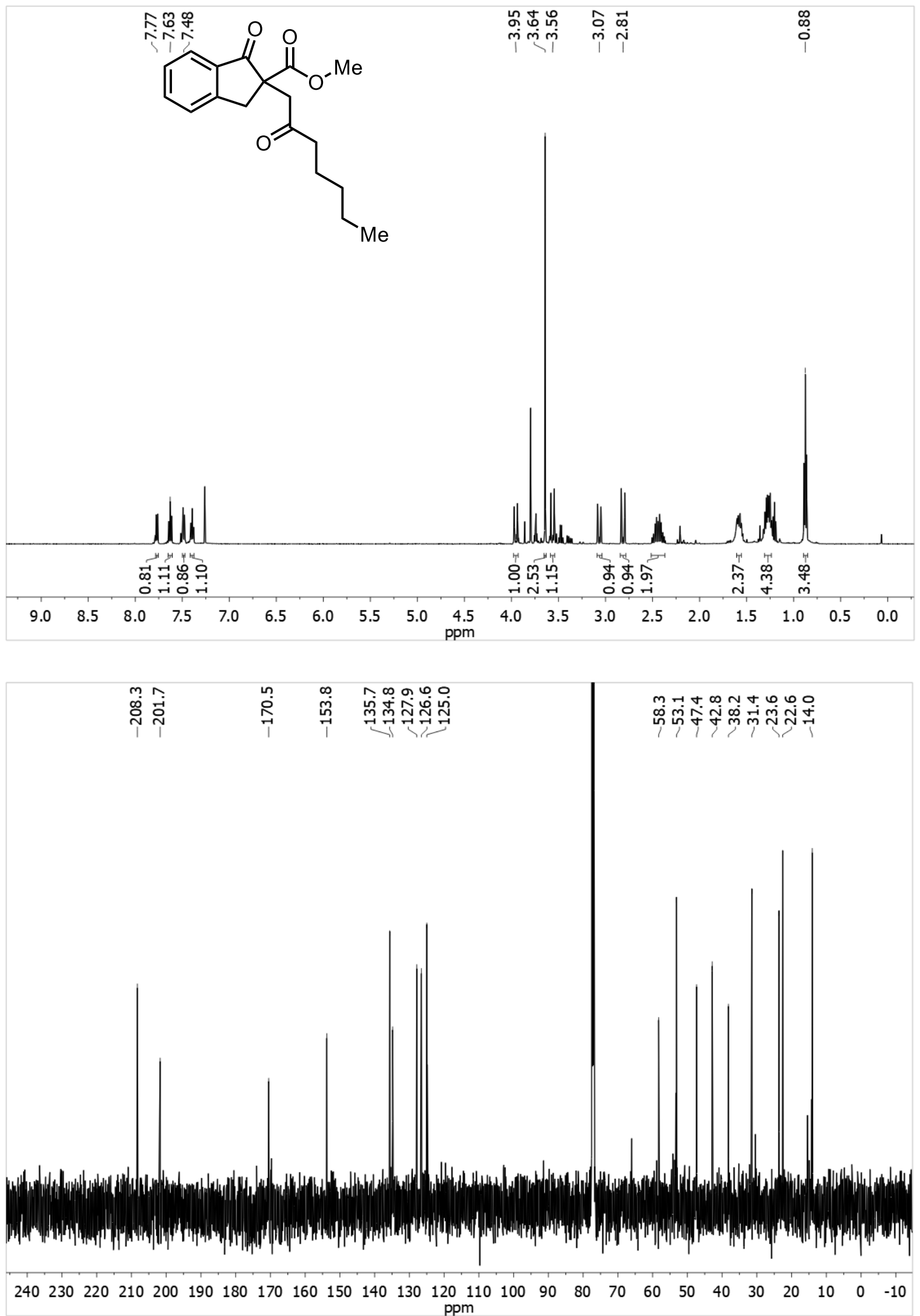
methyl 2-(3,3-dimethyl-2-oxobutyl)-1-oxo-2,3-dihydro-1H-indene-2-carboxylate (20)
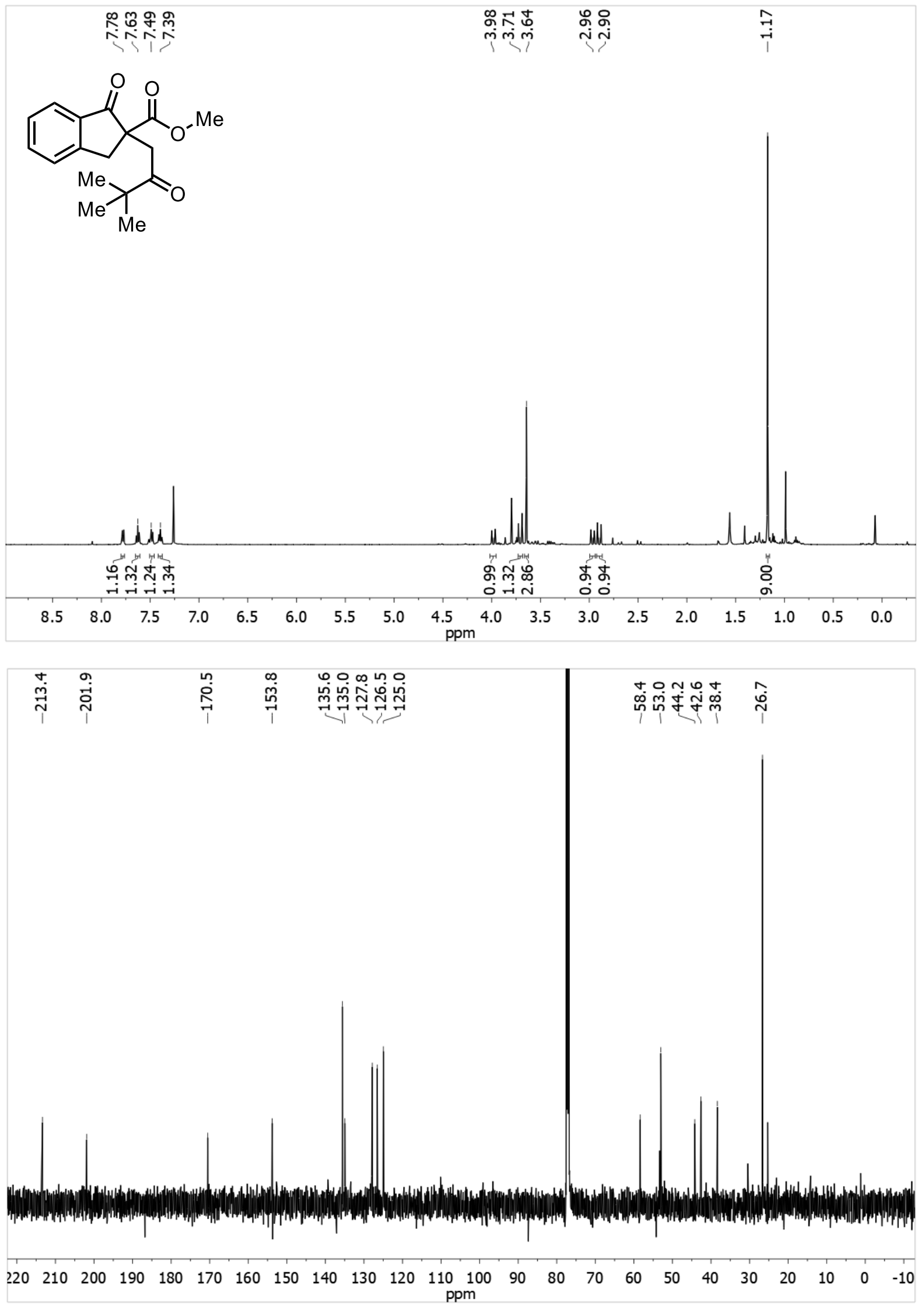
ethyl 1-(3,3-dimethyl-2-oxobutyl)-2-oxocyclopentane-1-carboxylat (2q)
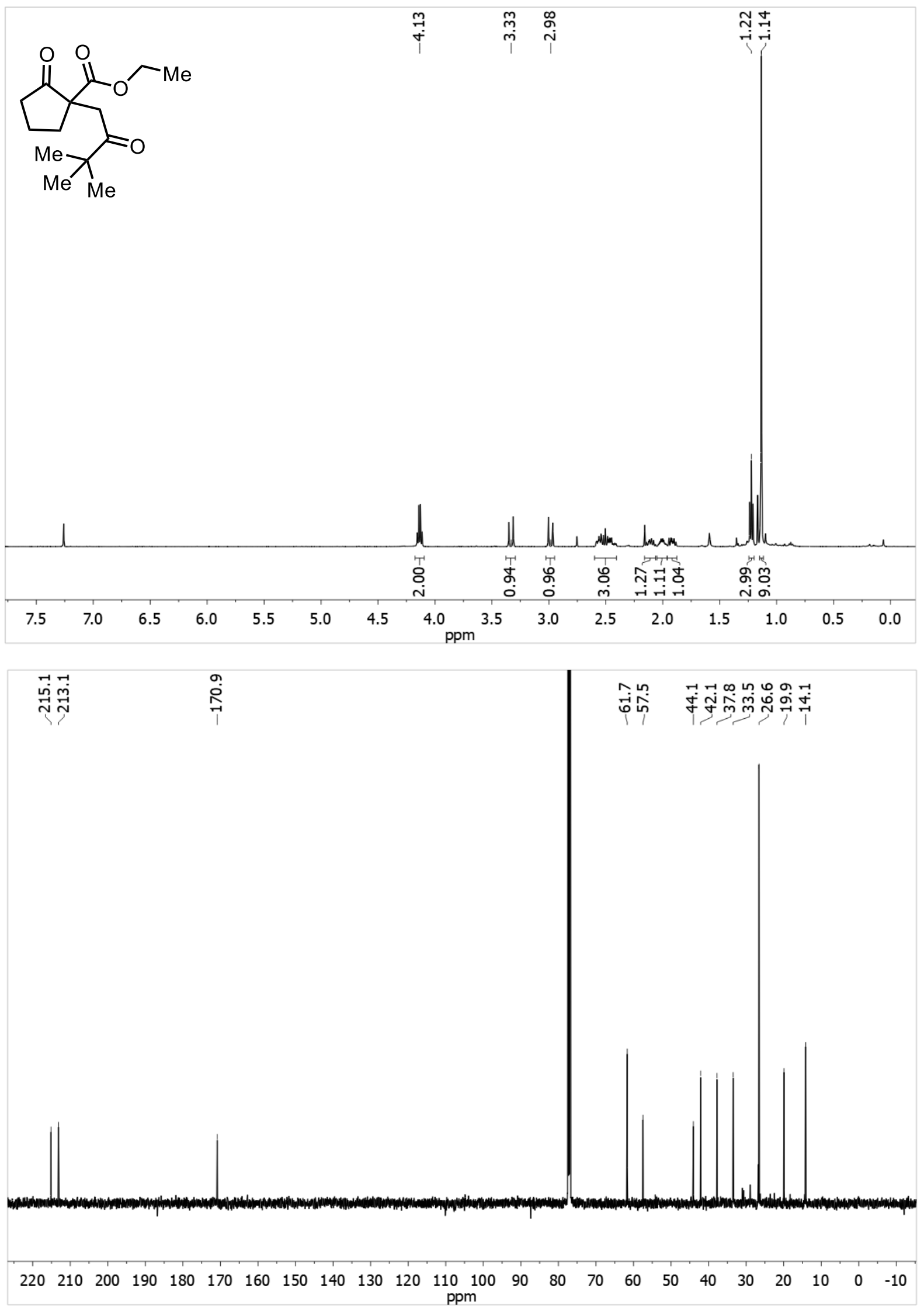
ethyl-2-oxo-1-(2-oxocyclohexyl)cyclopentane-1-carboxylate (2r):
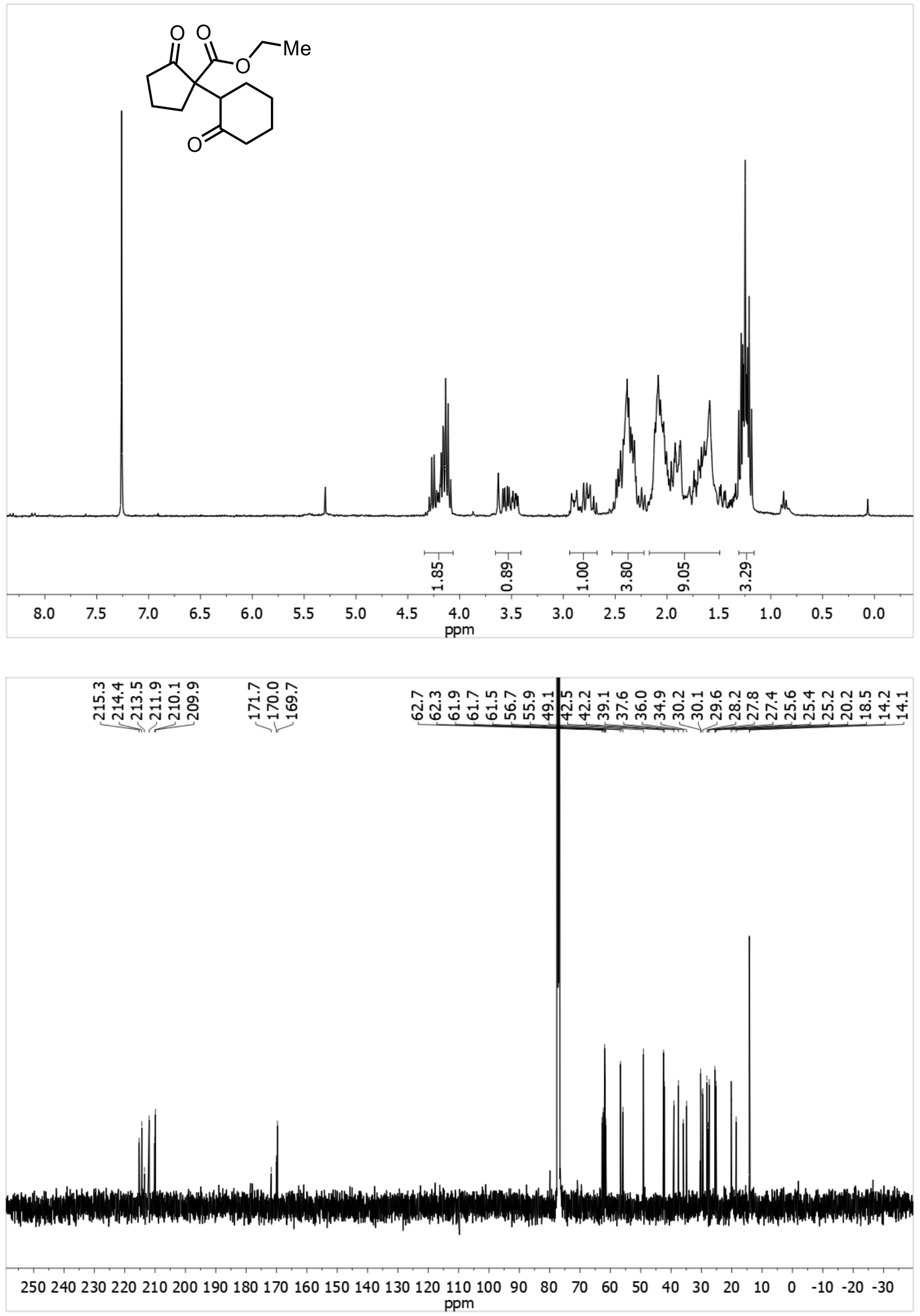
methyl 1-oxo-2-(2-oxo-2-phenylethyl)2,3-dihdro-1 $H$-indene-2-carboxylate (2s)
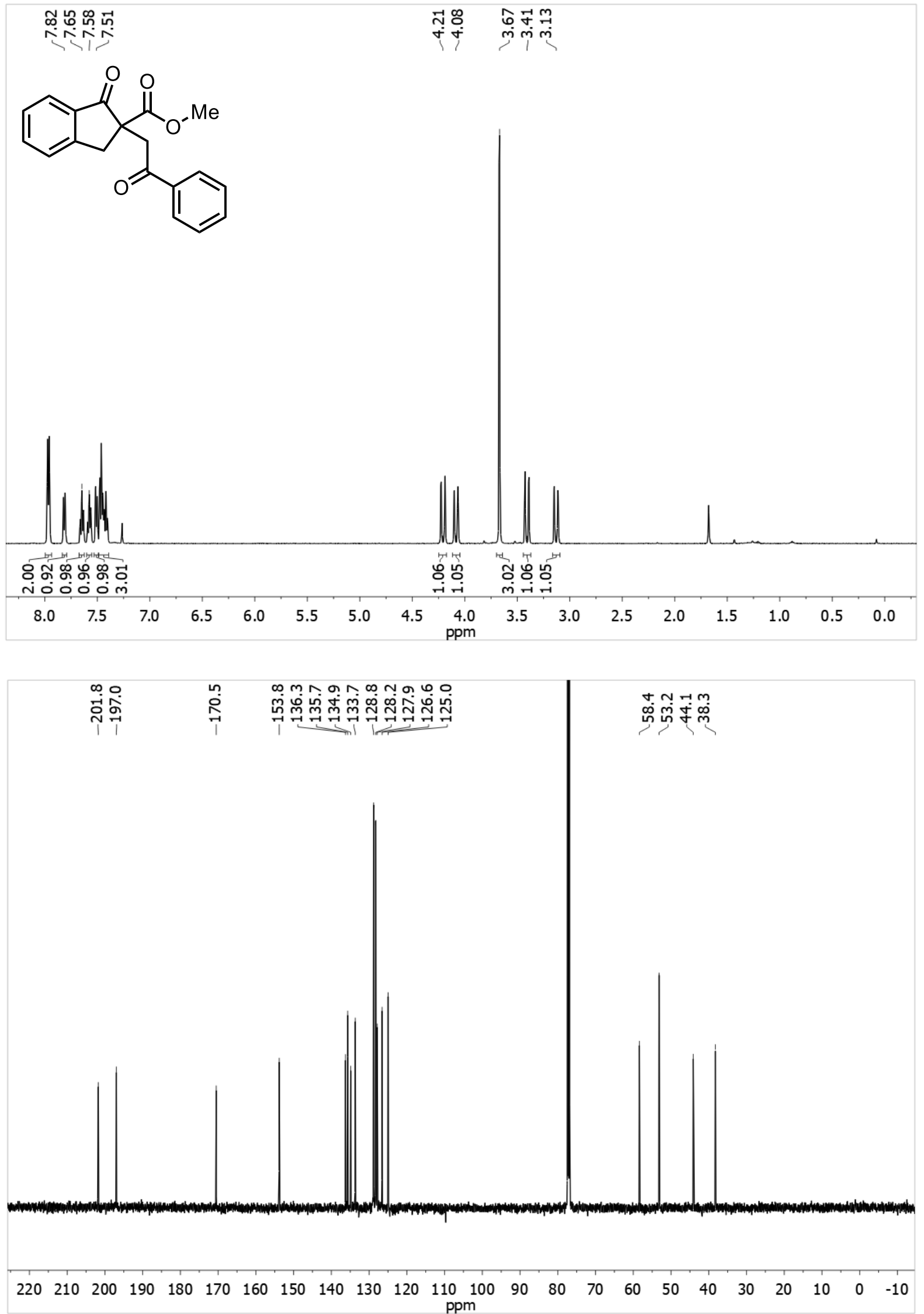
ethyl 2-oxo-1-(3-oxobutyl)cyclopentane-1-carboxylate (2t)
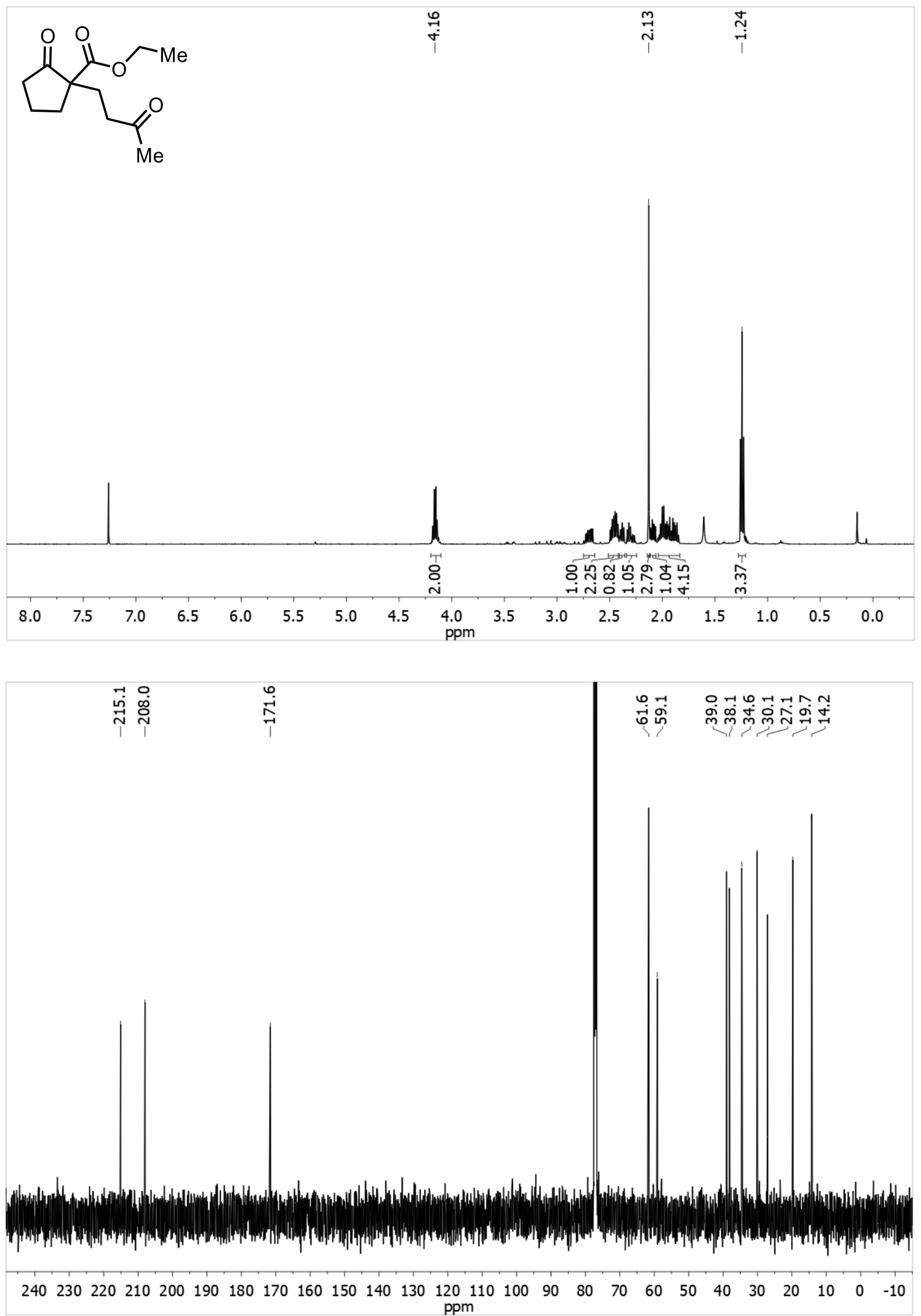
methyl 1-oxo-2-(3.oxobutyl)-2,3-dihydro-1 $\mathrm{H}$-indene-2-carboxylate (2u)
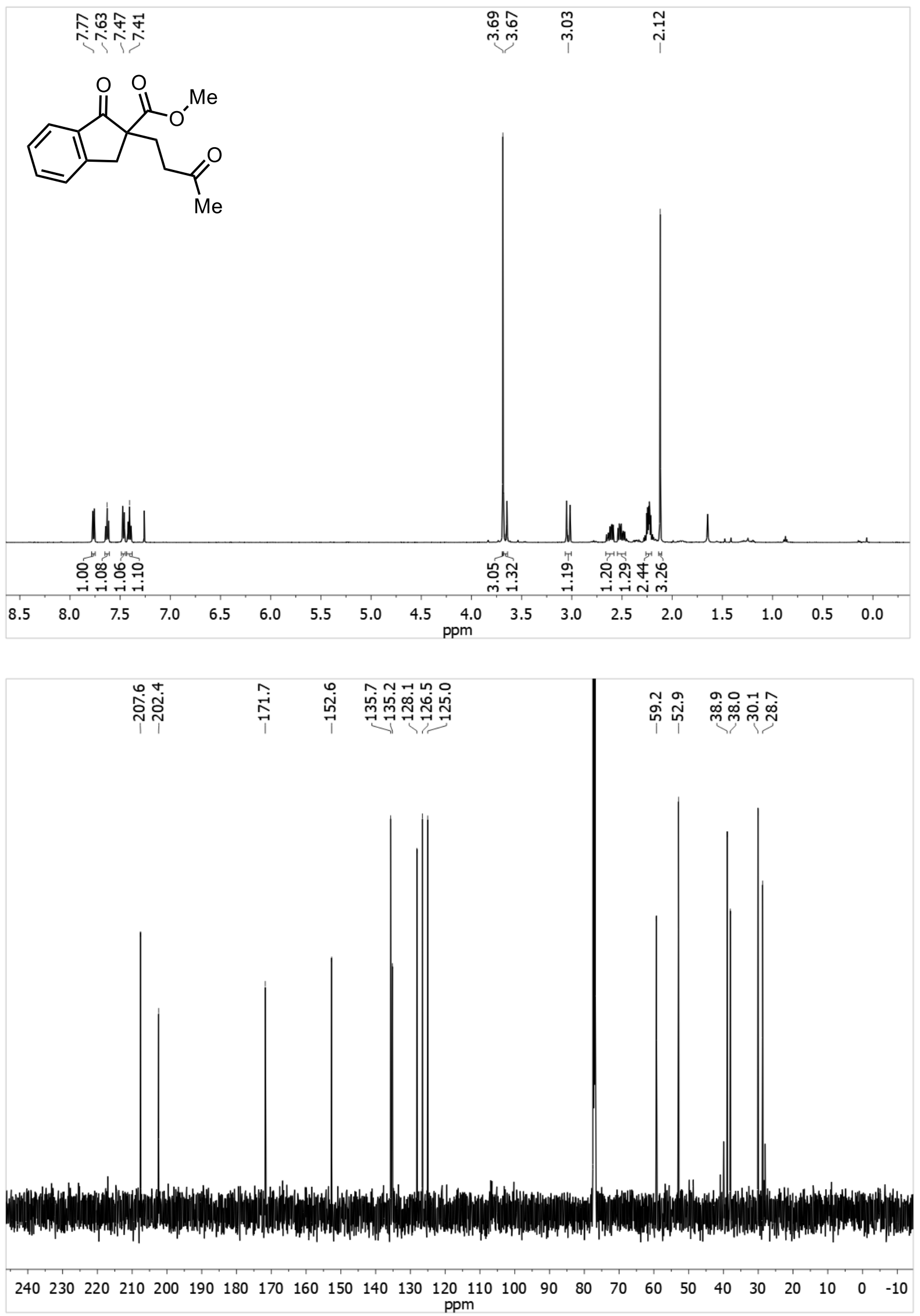
tris(pyridine-2-ylmethyl)amine

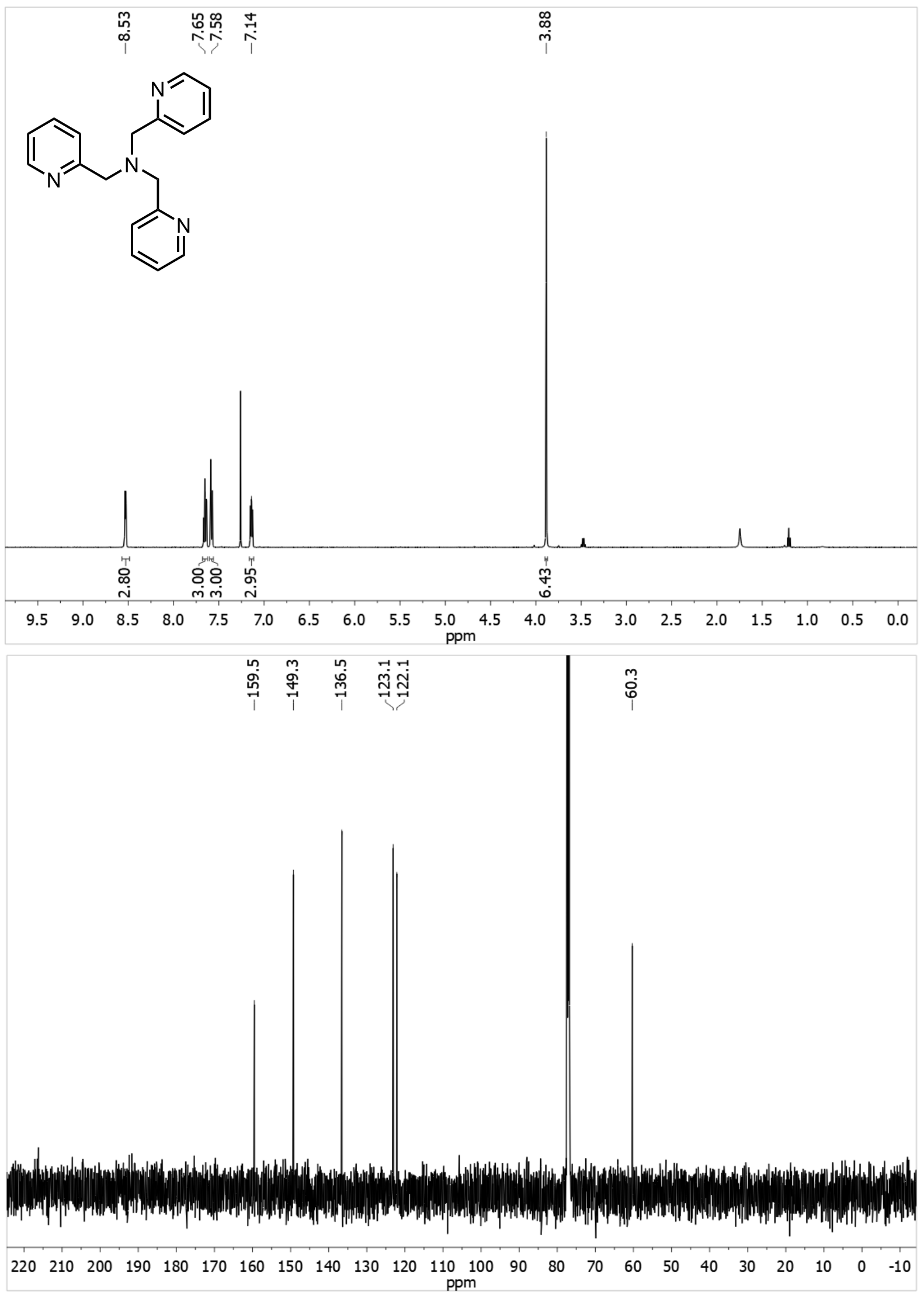


3,7-bis((R)-1-phenylethyl)-3,7-diaza-1,5(2,6)-dipyridinacyclooctaphane (8)
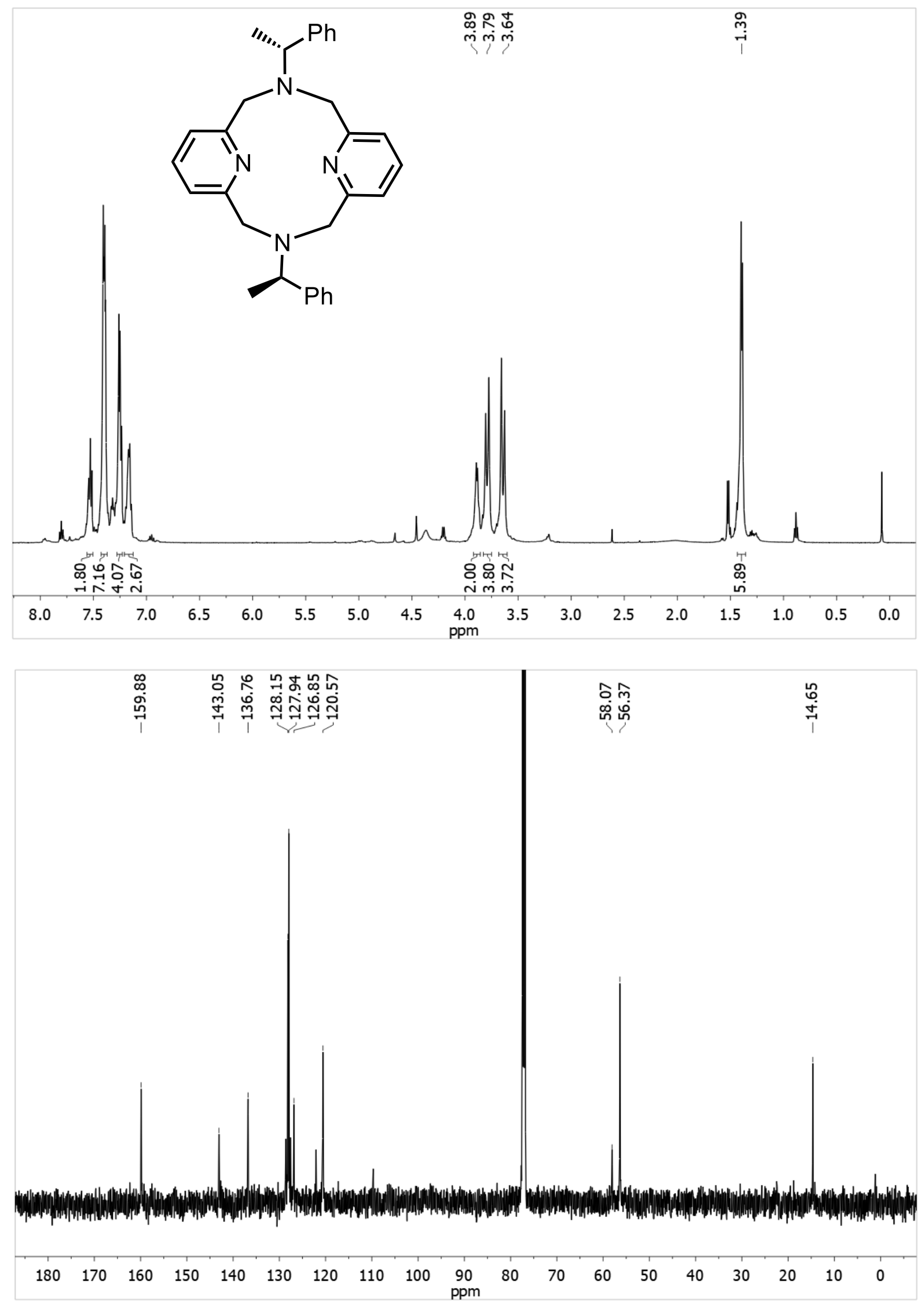


\section{References}

[1] Fulmer, G. R.; Miller, A. J. M.; Sherden, N. H.; Gottlieb, H. E.; Nudelman, A.; Stoltz, B. M.; Bercaw, J. E.; Goldberg, K. I. Organometallics 2010, 29, 2176-2179.

[2] Walshe, N. D. A.; Goodwin, G. B. T.; Smith, G. C.; Woodward, F. E. Org. Synth. 1987, $65,1$.

[3] Cazeau, P.; Duboudin, F.; Moulines, F.; Babot, O.; Dunogues, J. Tetrahedron 1987, 43, 2075-2088.

[4] Esumi, N.; Suzuki, K.; Nishimoto, Y.; Yasuda, M. Org. Lett. 2016, 18, 5704-5707.

[5] Buckley, B. R.; Fernández D.-R, B. Tetrahedron Lett. 2013, 54, 843-846.

[6] Novák, L.; Kolonits, P.; Szántay, C.; Aszódi, J.; Kajtár, M. Tetrahedron 1982, 38, $153-$ 159.

[7] Haraguchi, R.; Ikeda, Z.; Ooguri, A.; Matsubara, S. Tetrahedron 2015, 71, 8830-8837.

[8] Lemus, C.; Poleschak, M.; Gailly, S.; Desage-El Murr, M.; Koch, M.; Deguin, B. Chem. Eur. J. 2013, 19, 4686-4890.

[9] Nagata, T.; Matsubara, H.; Kiyokawa, K.; Minakata, S. Org. Lett. 2017, 19, 4672-4675.

[10] Geibel, I.; Christoffers, J. Eur. J. Org. Chem. 2016, 918-920.

[11] Gu, X.-P.; Okuhara, T.; Ikeda, I.; Okahara, M. Synthesis 1988, 535-537.

[12] Pflantz, R.; Tielmann, P.; Rössle, M.; Hoenke, C.; Christoffers, J. Eur. J. Org. Chem. 2007, 3227-3238.

[13] Brazel, C.; Dupre, N.; Malacria, M.; Hasenknopf, B.; Lacote, E.; Thorimbert, S. Chem. Eur. J. 2014, 20, 16074-16077.

[14] Singh, C.; Gangwar, M. K.; Ghosh, P. Inorg. Chim. Acta 2017, 466, 358-369.

[15] Akiyama, T.; Katoh, T.; Mori, K. Angew. Chem. Int. Ed. 2009, 48, 4226-4228.

[16] Patil, P. H.; Filonenko, G. A.; Lapointe, S.; Fayzullin, R. R.; Khusnutdinova, J. R. Inorg. Chem. 2018, 57, 10009-10027.

[17] Mondal, A. K.; Jover, J.; Ruiz, E.; Konar, S. Chemistry 2017, 23, 12550-12558.

[18] Hitomi, Y.; Ando, A.; Matsui, H.; Ito, T.; Tanaka, T.; Ogo, S.; Funabiki, T. Inorg. Chem. 2005, 44, 3473-3478.

[19] Duboc, C.; Phoeung, T.; Zein, S.; Pecaut, J.; Collomb, M. N.; Neese, F. Inorg. Chem. 2007, 46, 4905-4916.

[20] Takai, K.; Ohno, A., Hino, M.; Shitaoka, T.; Komeyama, K.; Yoshida, H. Chem. Commun. 2014, 50, 12285-12288.

[21] Koch, D.; Schäfer, H.; Steckhan, E. Chem. Ber. 1974, 107, 3640-3657.

[22] Barba, F.; Desamparados, V.; Guirado, A. Synthesis 1984, 7, 593-595.

[23] Zhu, X.; Lin, Y.; Sun, Y.; Beard, M. C.; Yan, Y. J. Am. Chem. Soc. 2019, 141, 733-738. 